\title{
Geotemporospatial and causal inference epidemiological analysis of US survey and overview of cannabis, cannabidiol and cannabinoid genotoxicity in relation to congenital anomalies 2001-2015
}

Albert Stuart Reece ${ }^{1,2^{*}}$ and Gary Kenneth Hulse $e^{1,2}$

\begin{abstract}
Background: Cannabinoids including cannabidiol have recognized genotoxic activities but their significance has not been studied broadly epidemiologically across the teratological spectrum. We examined these issues including contextual space-time relationships and formal causal inferential analysis in USA.

Methods: State congenital anomaly (CA) rate (CAR) data was taken from the annual reports of the National Birth Defects Prevention Network 2001-2005 to 2011-2015. Substance abuse rates were from the National Survey of Drug Use and Health a nationally representative longitudinal survey of the non-institutionalized US population with $74.1 \%$ response rate. Drugs examined were cigarettes, monthly and binge alcohol, monthly cannabis and analgesic and cocaine abuse. Early termination of pregnancy for abortion (ETOPFA) rates were taken from the published literature. Cannabinoid concentrations were from Drug Enforcement Agency. Ethnicity and income data were from the US Census Bureau. Inverse probability weighted (IPW) regressions and geotemporospatial regressions conducted for selected CAs.
\end{abstract}

Results: Data on 18,328,529 births from an aggregated population of 2,377,483,589 for mid-year analyses 2005-2013 comprehending 12,611 CARs for 62 CAs was assembled and ETOPFA-corrected (ETOPFACAR) where appropriate. E-Values for ETOPFACARs by substance trends were elevated for THC (40 CAs), cannabis (35 CAs), tobacco (11 CAs), cannabidiol (8 CAs), monthly alcohol (5 CAs) and binge alcohol (2 CAs) with minimum E-Values descending from $16.55,1.55 \times 10^{7}, 555.10,7.53 \times 10^{19}, 9.30$ and 32.98. Cardiovascular, gastrointestinal, chromosomal, limb reductions, urinary, face and body wall CAs particularly affected. Highest v. lowest substance use quintile CAR prevalence ratios $2.84(95 \%$ C.I. $2.44,3.31), 4.85(4.08,5.77)$ and $1.92(1.63,2.27)$ and attributable fraction in exposed $0.28(0.27,0.28), 0.57$ $(0.51,0.62)$ and $0.47(0.38,0.55)$ for tobacco, cannabis and cannabidiol. Small intestinal stenosis or atresia and obstructive genitourinary defect were studied in detail in lagged IPW pseudo-randomized causal regressions and spatiotemporal models confirmed the causal role of cannabinoids. Spatiotemporal predictive modelling demonstrated

${ }^{*}$ Correspondence: stuart.reece@bigpond.com

${ }^{2}$ School of Medical and Health Sciences, Edith Cowan University,

Joondalup, WA 6027, Australia

Full list of author information is available at the end of the article

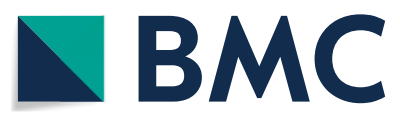

(c) The Author(s) 2022. Open Access This article is licensed under a Creative Commons Attribution 4.0 International License, which permits use, sharing, adaptation, distribution and reproduction in any medium or format, as long as you give appropriate credit to the original author(s) and the source, provide a link to the Creative Commons licence, and indicate if changes were made. The images or other third party material in this article are included in the article's Creative Commons licence, unless indicated otherwise in a credit line to the material. If material is not included in the article's Creative Commons licence and your intended use is not permitted by statutory regulation or exceeds the permitted use, you will need to obtain permission directly from the copyright holder. To view a copy of this licence, visit http://creativecommons.org/licenses/by/4.0/. The Creative Commons Public Domain Dedication waiver (http://creativeco mmons.org/publicdomain/zero/1.0/) applies to the data made available in this article, unless otherwise stated in a credit line to the data. 
strongly sigmoidal non-linear cannabidiol dose-response power-function relationships $\left(P=2.83 \times 10^{-60}\right.$ and $1.61 \times 10^{-71}$ respectively).

Conclusions: Data implicate cannabinoids including cannabidiol in a diverse spectrum of heritable CAs. Sigmoidal non-linear dose-response relationships are of grave concern.

These transgenerational genotoxic, epigenotoxic, chromosomal-toxic putatively causal teratogenic effects strongly indicate tight restrictions on community cannabinoid penetration.

Keywords: Cannabis, Cannabinoid, $\triangle 9$-tetrahydrocannabinol, Cannabigerol, Cannabidiol, Mechanisms, Congenital anomalies, Teratogenesis, Genotoxicity, Epigenotoxicity, Limb reduction deficiencies, Leg reduction deficiencies,

Chromosomal toxicity, Multigenerational genotoxicity, Transgenerational teratogenicity

\section{Background}

Both "Epidiolex" (cannabidiol) registered in the USA by the Food and Drug Administration (FDA) and Sativex ( $\Delta$ 9-tetrahydrocannabinol (THC) - cannabidiol) registered by the Medicines and Healthcare Products Regulatory Authority (MHRA) of the United Kingdom carry strong warnings on their Product Information and Prescribers Information leaflets against their use in pregnancy and breast feeding which is the standard warning for genotoxic effects which routinely accompanies medicines including cytotoxic and cancer agents $[1,2]$. Similar warnings occur on the labelling of "Hemp Oil" which is made freely accessible to the Australian public on supermarket shelves. Such overt warnings relating to acknowledged genotoxicity by the distributors and marketers of cannabinoids, and mandated warnings required by official drug regulators on both sides of the Atlantic directly imply that the genotoxicity of these agents is acknowledged in laboratory and preclinical studies and is in truth an established fact of science.

Paradoxically what might be termed the "standard" or "establishment" view of the risks posed by the use of cannabinoid products in pregnancy is relatively benign. Major authorities and several smaller convenience sample series claim that the use of cannabis in pregnancy is associated with increased prematurity, smaller head circumference, increased small for gestational age, low birth weight and relative infertility in male and female users [3-5]. This view which enjoys widespread currency in the medical profession, is clearly at odds with the official governmental view endorsed in the requirements on registered product information for the medical profession and consumers, but is nevertheless typical of the communitywide confusion relating to much of the information on cannabis and cannabinoids.

A broader and more concerning view on cannabinoid teratogenicity is expressed by other authorities including the Centres for Disease Control (CDC) Atlanta, Georgia, the American Heart Association (AHA) and the American Academy of Paediatrics (AAP) who have together warned of increased rates of six birth defects after prenatal cannabis exposure including ventricular septal defect, Epsteins anomaly, gastroschisis, diaphragmatic hernia, oesophageal atresia with or without tracheoesophageal fistula and anencephalus [6-8]. The American College of Obstetricians and Gynaecologists (ACOG) strongly warn against the use of cannabis products in pregnancy [9]. Longitudinal studies of neurological and psychomotor development in prenatally exposed children conducted in Pittsburgh, Toronto and Netherlands uniformly indicate worrying levels of autism-like and ADHD-like features with altered neurological development and impairments of emotional development, motor tone and fine motor skills and cortical executive and visuospatial processing [10].

The most useful experimental animal models in which to study the effects of prenatal drug exposure are New Zealand white rabbits and hamsters. Classical studies from 1969 performed in rodents and hamsters showed a variety of defects including limb reduction, exencephaly, spina bifida, omphalocele, multiple malformations and myelocoele $[11,12]$. As was noted at the time "this is a formidable list" [12]. However clinical confirmation of such a concerning and wide-ranging spectrum of congenital anomalies was mostly lacking. In 2007 a novel report from Hawaii listed 21 birth defects as being elevated after prenatal cannabis exposure, particularly affecting the cardiovascular, gastrointestinal, urinary and chromosomal systems and including arm defects, syndactyly and polydactyly however this study remained very much an exception and outlier for many years [13].

In an historical case series of illicit poly-drugs users from Washington DC 148 pregnancies amongst 140 women produced 12 embryos or infants with major congenital abnormalities, $43 \%$ had spontaneous first trimester abortions and four of eight serial pregnancies produced infants or embryos with major abnormalities [14]. The major congenital anomaly rate was a calculated by the authors at 96/1000 live births or 16 times the then control rate in USA in 1972 [14]. The usually quoted rate for spontaneous abortions at that time in USA was up to $20 \%$. Of the eight infants whose major congenital 
anomaly was listed six had neural tube closure defects (meningomyelocele, myelocele, spina bifida or hydrocephalus), one had a cardiovascular defect (Tetralogy of Fallot), one had neuroblastoma and one had limb abnormalities (absent feet, absent finger and absent phalanges from fingers). All patients smoked cannabis [14, 15].

A report on atrial septal defect secundum type from the CDC database showing much higher rates and a steep acceleration of the rate of increase of atrial septal defect in high cannabis use states in the USA in recent years appeared which carried two major corollaries [16]. Firstly it implied that the list of cardiovascular anomalies jointly proposed by the AAP and AHA was incomplete. Secondly it implied that our knowledge of the subject of clinical cannabinoid teratogenesis including the list of cannabis-related congenital anomalies was similarly incomplete.

The concerning Hawaiian study has since been supported by studies from other locations. Confirmation of the experimentally identified spina bifida and encephalocele findings recently came from an analysis of Canadian data [17]. Indeed total congenital anomalies, particularly including cardiovascular defects and chromosomal anomalies were recently noted to be three times higher in the northern Territories of Canada which traditionally smoke two to three times as much cannabis as Canadians living in the south [18]. An Australian report showed that 18 congenital defects were higher in high cannabis using parts of Northern New South Wales [19]. Colorado was noted to have a $29 \%$ jump in the expected rate of total birth defects across the period of cannabis legalization 2000-2013 and included particularly cardiovascular, central nervous system, genitourinary, musculoskeletal and chromosomal CAs [20].

Cannabinoids including cannabidiol have been implicated in direct damage by oxidation to DNA bases which is a major genotoxic and mutagenic lesion [21]. They have long been known to be toxic to chromosomes which are the natural way in which DNA is packaged inside the cell nucleus [22]. It was shown long ago that cannabinoids reduce the synthesis of the major molecules of biology DNA, RNA proteins and histones [23-34]. Such gross level changes necessarily impact the genomic code. Translated into a twenty-first century understanding this would imply major interference in the epigenetic code where genome accessibility, controlled by histone modifications, the formation of euchromatin and the assembly of topologically organized transcriptionally active domains (the chromosomal "A compartment") within the nucleus constitutes a major portion of normal gene regulation, cell function and indeed epigenetic cell specification and lineage determination [35]. And it has been well established that cannabinoids carry a heavy epigenetic footprint which is inheritable for several subsequent generations [35-41].

As was recently observed chromosomal toxicity, genotoxic and epigenotoxic lesions can reasonably be expected to manifest in congenital anomaly profiles and patterns of cancerogenesis [42]. What is clearly lacking in the literature is a genotoxic survey of a national teratological database to study the issue of patterns of teratogenesis as they relate to substance exposure. The application of the formal techniques of geospatial analysis and causal inferential analysis to the whole database tracked by CDC of 62 birth defects is a massive task which can only be commenced in this forum. It is therefore our purpose in the present paper to present an overview and introduction to this topic with a few teratological case examples to illustrate the way in which such studies can be extended and the power of these analytical techniques. Formal treatment of the whole field must be left for another occasion. Since the required teratological and substance exposure and related data is available for USA that nation has been chosen for the present investigation.

As has been pointedly observed it is vitally important in any review of teratological epidemiology to consider the impact of early termination of pregnancy for anomalies (ETOPFA) [43, 44]. Our study provides estimates of these ETOPFA practices which are used to complete applicable datasets for affected congenital anomalies (CAs).

Given the rapid increase in the penetration of cannabis and cannabinoids into modern American society, all studies related to cannabinoid teratogenesis and cannabinoid genotoxicity must be regarded as urgent and of high priority in the national research agenda.

A related concern is the potential for cannabinoids to enter the food chain. Cases of babies born without limbs have been noted in France and Germany where cannabis has become widely available [45-50] however this has not been seen in nearby Switzerland where its entry into the food chain is not permitted. Rapid introduction of cannabis into Colorado recently was associated with a $29 \%$ jump in total congenital anomalies [20] and Kentucky saw a massive and sharp spike in the incidence of atrial septal defect in recent years as cannabis has increasingly replaced tobacco as a major cash crop [16].

Not since Distillers unleashed thalidomide on the global market in 1957 has an agent which is known to be genotoxic been aggressively marketed for commercial reasons [51]. Of note the thalidomide debacle was avoided in the USA primarily because of genotoxic concerns $[52,53]$. This international tragedy of recent history is also the foundational reason for the development of the modern drug regulatory scheme in many nations [53].

Aside from the fact of cannabis mutagenicity and genotoxicity itself one of the aspects of this subject which 
we find of most concern is the clear replication in many predictive geotemporospatial models of a sigmoidal relationship between cannabidiol and cannabinoid exposure and teratogenic outcomes for many congenital anomalies which is clearly highly reminiscent of the exponential dose-response relationships observed in numerous in vitro studies of cannabinoid genotoxicity and mitochondriopathy-epigenotoxicity [24, 26, 31, 54-65]. It is the non-linear power function of dose-response between increased cannabinoid exposure and teratological outcomes which must be of particular concern to any community moving into a higher cannabinoid exposure zone. Equally of concern an exponential relationship was observed in both actual and predicted modelled trend studies of the relationship between cannabinoid exposure and US autism incidence [66]. Taken together such findings imply exponentiation both of major neurotoxic and major genotoxic developmental outcomes.

It is self-evident that with the endocannabinoids playing critical roles in many body systems drugs modulating the endocannabinoid system will increasingly enter the international therapeutic marketplace in the coming years. We also feel that in order to assist cannabinoid therapeutics to find their appropriate niche in the global market that a proper understanding and appreciation of their long term neurotoxic and genotoxic activities is an absolute requirement both for regulators and for the public at large so that intergenerational community safety continues to be prioritized as a central and principal concern.

The overall purpose of the present analysis was to investigate substance and particularly cannabinoid exposure as a putative environmental risk factor for the observed spectrum of congenital anomalies. This was done directly using ecological USA data in bivariate analysis of continuous covariates. Key epidemiological parameters of public health interest such as the prevalence ratio, the aetiological fraction in the exposed and the population attributable risk were calculated from an analysis of categorized data. Detailed multivariable regression was undertaken using inverse probability weighted mixed effects, robust and panel regression for two selected CAs and spatiotemporal regression was also conducted for these CAs. Extensive use of the formal techniques of causal inference namely E-Values and inverse probability weighting was engaged to correct for the ecological fallacy and convert data into a pseudo-randomized quasi-experimental design. Finally predictive mathematical modelling was conducted to study overall trends of selected CAs as a function of cannabinoid exposure.

The minimum E-Value indicates the minimum strength of association required of some extraneous confounder covariate with both the outcome of interest and the exposure of concern to explain an observed assocation [67-69]. It plays a central role in formal epidemiological assignment of causal relationships.

An overview and survey of a geospatial consideration of the field of genotoxicity manifested as cancerogenesis is the subject of a series of companion papers.

\section{Methods}

Data

Rates of birth defects were taken from the annual reports of the National Birth Defects Prevention Network (NBDPN) 2001-2005 to 2011-2015 which is coordinated from the Centres for Disease Control (CDC), Atlanta, Georgia. For the purposes of conducting the analysis the nominal year of the report was taken as the temporal midpoint of the year of the report. Hence for the most recent report we used which was 2011-2015 [70] the nominal year for analysis was 2013. We analyzed all the major CAs collected long term by NBDPN across this period totally 62 CAs. This was joined with annual USA state based drug use cross-tabulation data from the National Survey of Drug Use and Health (NSDUH) Substance Use and Mental Health Data Archive (SAMHDA) Restricted-Use Data Analysis System (RDAS) maintained by the Substance Abuse and Mental Health Services Administration (SAMHSA) [71]. The drugs of interest were last month cigarette use, last month alcohol use, last year binge alcohol use, last year non-medical use of opioid analgesia (Analgesics), last month use of cannabis and last year use of cocaine. Substance exposure was also considered as a categorical variable. This was facilitated by establishing substance exposure quintiles for each year with the first quintile representing the lowest exposure and the fifth quintile the highest exposure. The cannabinoid concentration in Federal cannabis seizures was taken from published reports of the Drug Enforcement agency [72-74]. Estimates of state level cannabinoid exposure was derived by multiplying the last month cannabis use rates by the Federal cannabinoid concentration. Quintiles for cannabinoid exposure were calculated across the whole period as a single group.

Some CAs and those particularly affecting chromosomal defects are heavily impacted by ETOPFA practice. The final ETOPFA rate by anomaly was arrived at as a composite synthesis of several published ETOPFA rates [75-82]. Moreover, as defined in at least one longitudinal annual time series of ETOPFA rates it seems highly likely that the ETOPFA rate has been incrementally increasing over time [83]. In the longitudinal time series the ETOPFA rate for Downs syndrome rose from low levels in 1980 to $70 \%$ in 2014 . This approximately linear rate of rise has been projected across all CAs according to the following formula: 
$E T O P F A \_R a t e=$ Reported_Rate $/(1-($ Composite $*$ FMaxTR $))$

where ETOPFA_Rate represents the adjusted CA rate, the Reported_Rate is the gazetted rate reported by NBDPN, the Composite rate is the composite rate derived from literature review shown in Table 1 and the FMaxTR is the Fraction of the Maximal Termination Rate in the year in question given in Supplementary Table 1 which is a tabular representation of graphical data taken from the only longitudinal series of ETOPFAs in the world we were able to identify [83].

Median household income and ethnicity data by state and year was sourced using tidycensus package [84] in $\mathrm{R}$ directly from the US Census bureau including linear interpolation for missing year data. The main ethnicities which were tracked included: Native Hawaiian / Pacific Islander (NHPI), American Indian / Alaska Native (AIAN), Asian-American, Hispanic-American, AfricanAmerican and Caucasian-American. Cannabinoid concentration data in USA at the Federal level was taken from published reports of the US Drug Enforcement Agency (DEA) [72-74]. The five cannabinoids of interest were $\Delta 9$-tetrahydrocannabinol (THC), cannabidiol (CBD), cannabigerol (CBG), cannabinol (CBN), and cannabichromene $(\mathrm{CBC})$. Federal cannabinoid concentration was multiplied by state level cannabis use to compute an estimate of cannabinoid exposure in each state.

Further technical details relating to statistical methodology are provided in an online Statistical Appendix.

\section{Data availability}

Data, including R-code, spatial weights, ipw weights and main source datasets has been made freely available through the Mendeley Data repository online and can be accessed at https://doi.org/10.17632/w6ks529sxd.1 .

\section{Ethics}

The University of Western Australia Human Research Ethics Committee granted ethical approval for this study on 7th January 2020 RA/4/20/7724.

\section{Results}

This section is set out in three sections. First we examine bivariate continuous associations. We then calculate key epidemiological parameters of interest from categorization of key exposure variables. We then demonstrate how inverse probability weighting can be employed in multivariable regression models and also use spatiotemporal models to investigate causal relationships formally and in a space-time context as an analytical pathway proof of concept for subsequent detailed studies across all congenital anomalies.
$18,328,529$ births occurred in USA in the eight nominal years 2005-2013. 2008 was omitted as CA data was not available for that year. The cumulative aggregated population of the USA for these eight years year-onyear was 2,377,483,589. 12,611 birth defect rates relating to 62 birth defects in the 50 states of the USA were extracted from the published reports of the National Birth Defects Prevention Network which is coordinated by the CDC. The defects of interest are listed in Supplementary Table 1 . The period of interest was 2005-2013 as that period could be related to drug and substance exposure data from the NSDUH from SAMHSA. Since NBDPN reports are issued for quinquennia this report comprehends the NBDPN reports from 2003-2007 to 2011-2015.

It is well known that several congenital anomalies are actively sought out by active antenatal screening programs. Some of these are subject to indications for early therapeutic termination of pregnancy for anomaly (ETOPFA). In considering the likely rate of congenital anomalies it is important to take this effect into consideration. Supplementary Table 1 also lists the ETOPFA rates from various published series [72-74]. Series were selected for their breadth of coverage of multiple congenital anomalies. The right hand column lists the ETOPFA rates applied in the present work which were a composite of these series. This estimate of the ETOPFA-corrected rate was a dependent variable of interest in some of the present analyses. Supplementary Table 2 shows the timedependent progression of the only longitudinal series of ETOPFA's we were able to identify which was the Down Syndrome ETOPFA rate in Western Australia [83].

\section{Continuous bivariate exposure survey}

Figure 1 shows the time dependent trajectories of these various CAs corrected for estimates of ETOPFA.

Figure 2 shows the substance exposure trends over this time period. Data was taken from the nationally representative annual SAMHSA NSDUH which reports a $74.1 \%$ response rate [85].

Figure 3 shows the annual estimated cannabinoid exposure for state level data estimated from Federal data from the DEA relating to cannabinoid concentrations in drug seizures and the state level last month cannabis consumption. Rising trends are noted for all cannabinoids except cannabidiol which is declining.

Figure 4 shows the relationship of the various ETOPFA-corrected CA rates (ETOPFACAR) to tobacco exposure. As is expected many show a rising and positive relationship.

Supplementary Fig. 1 shows the relationship of the ETOPFACAR estimates to binge alcohol exposure. Mostly weak or negative relationships are demonstrated. 


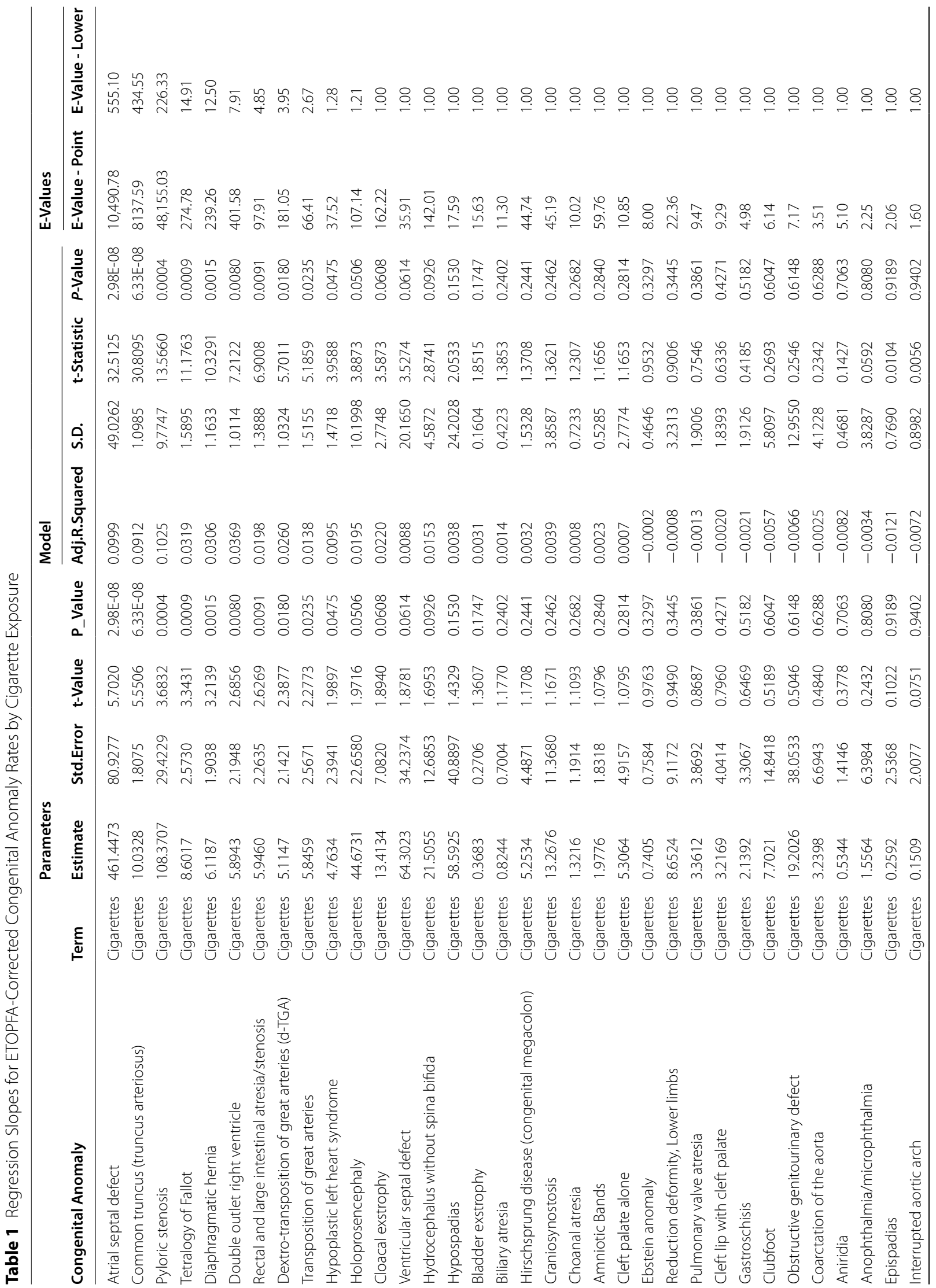




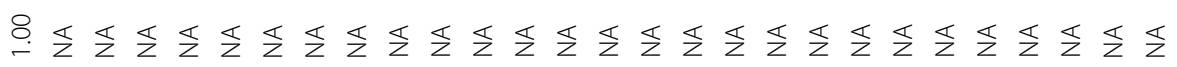

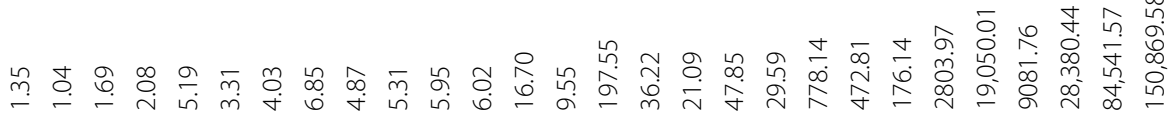

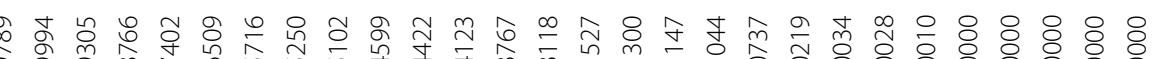

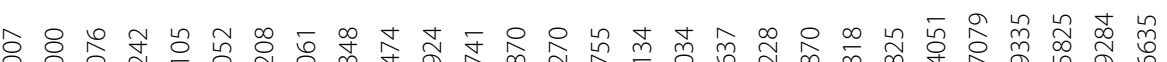

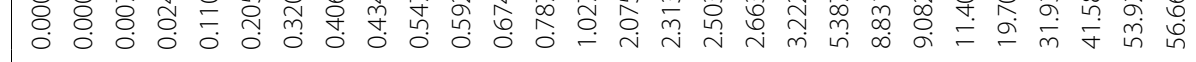

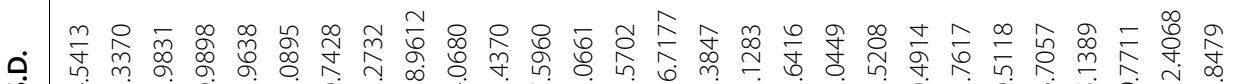

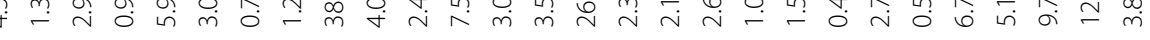

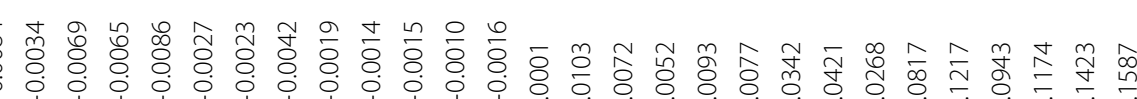

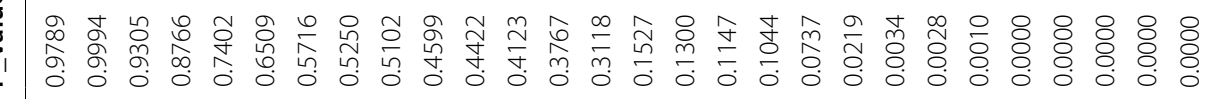

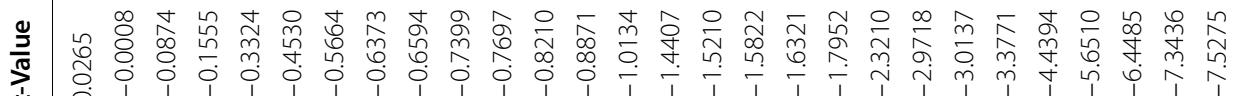
Z Ó

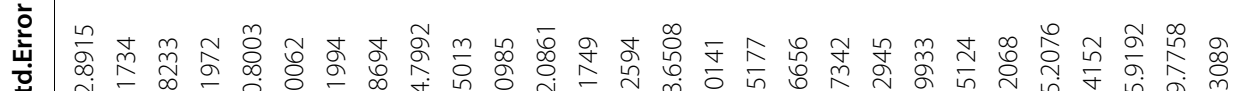
ஸ்

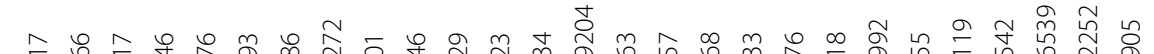

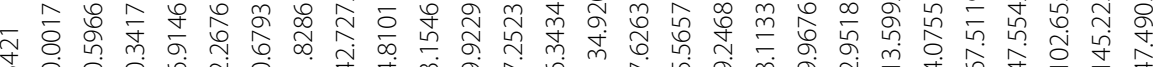
E E

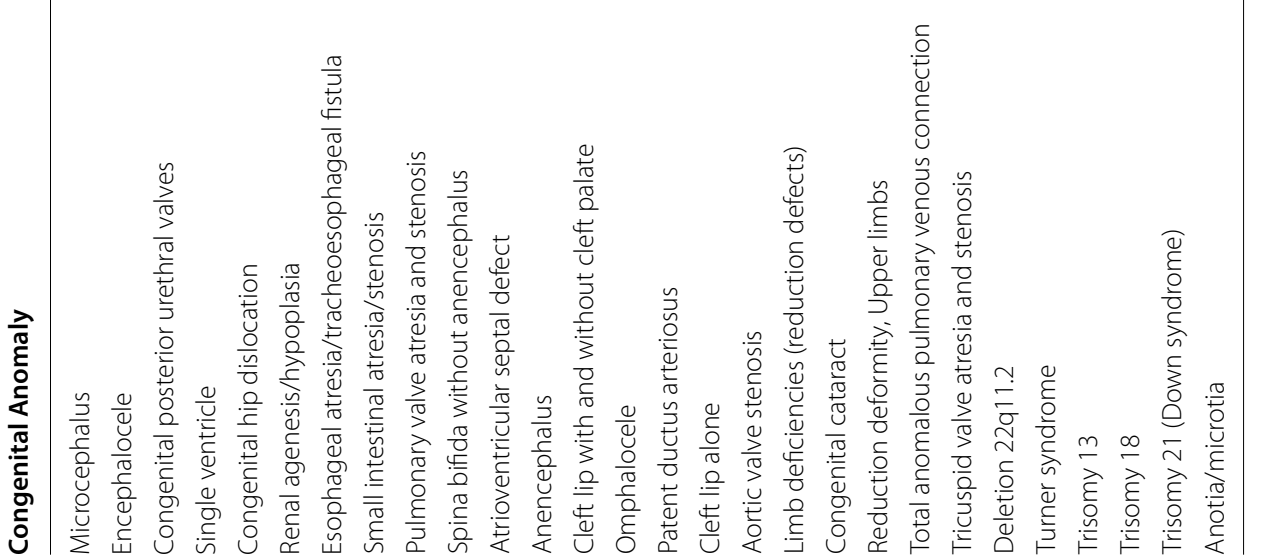




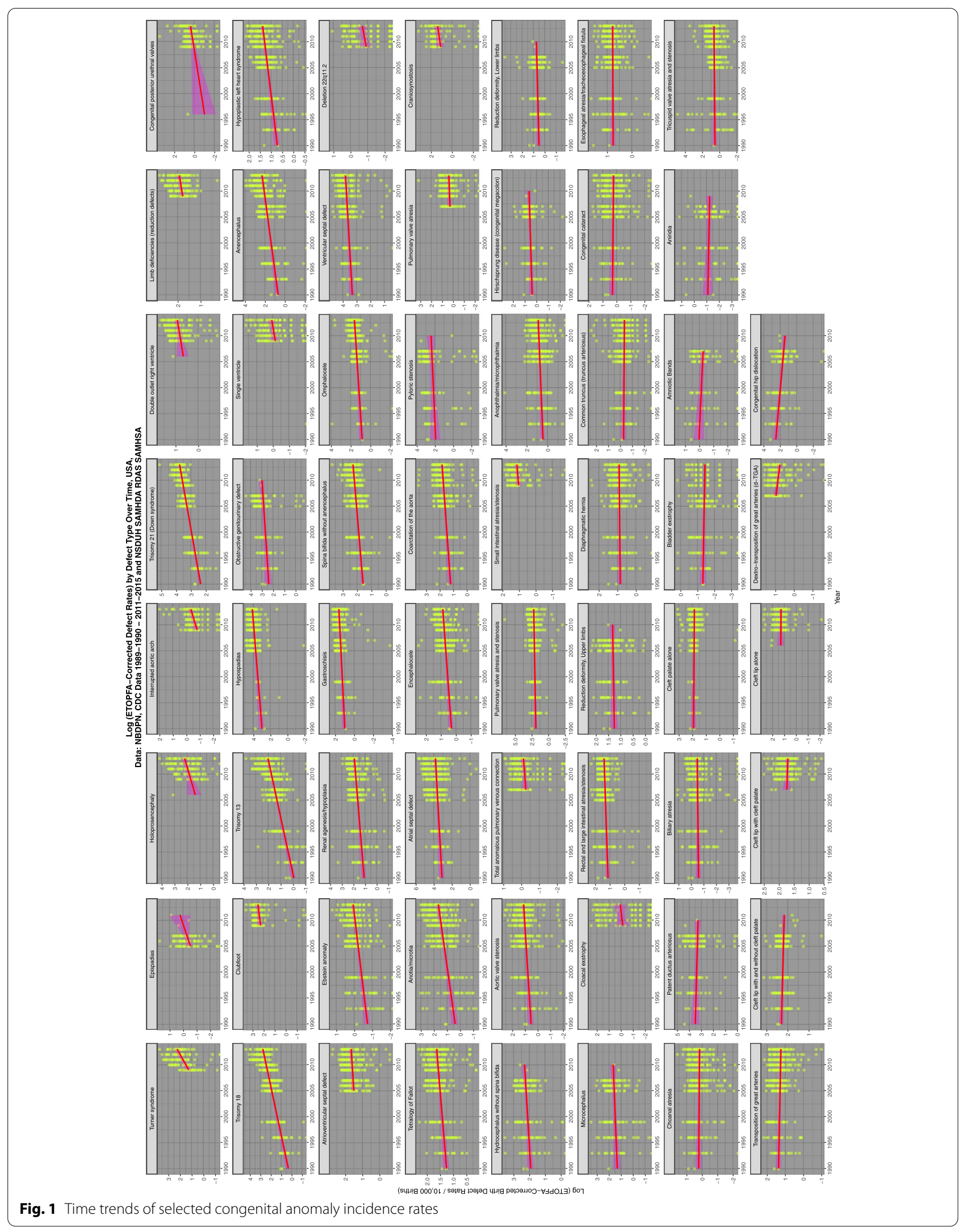




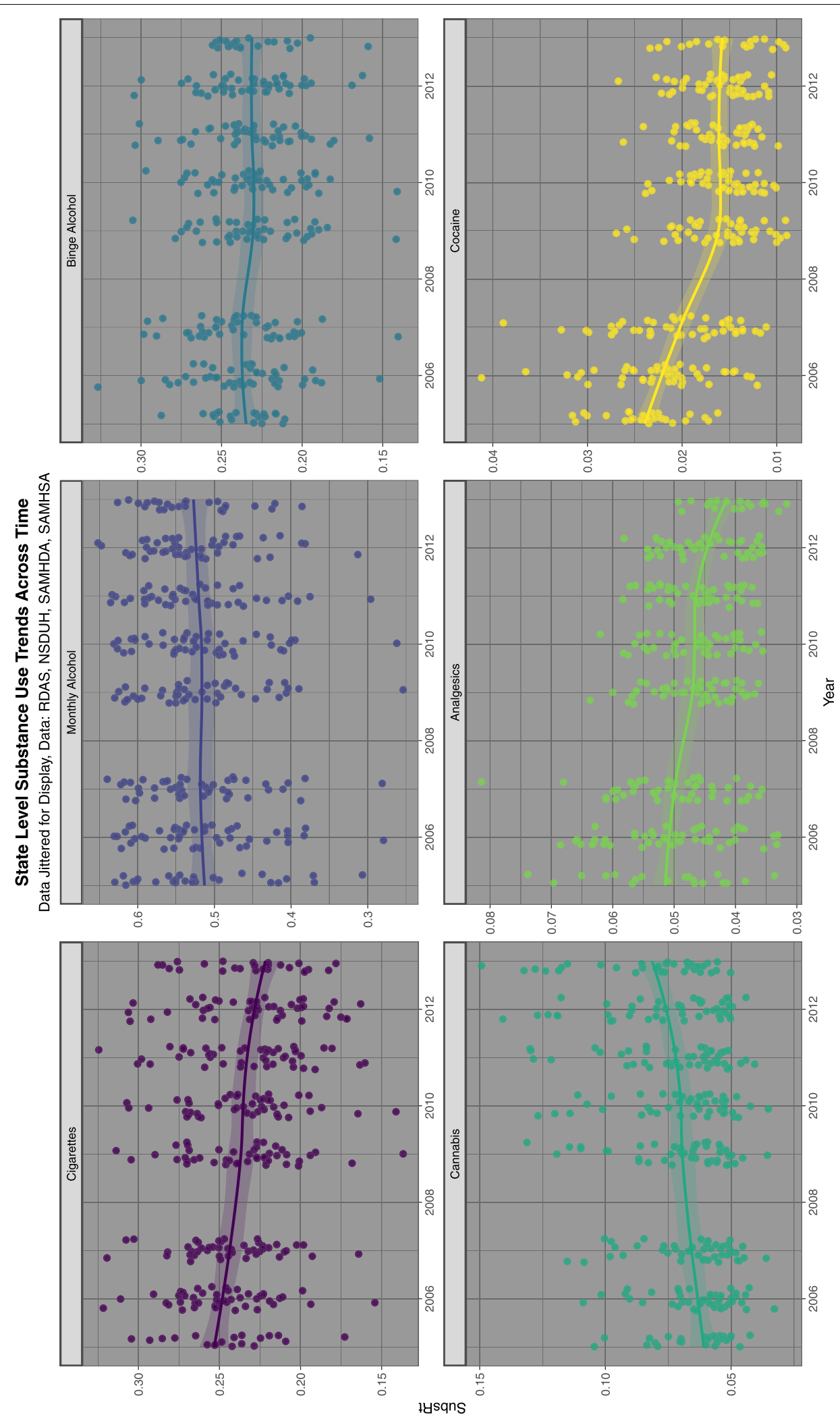

Fig. 2 Trends over time of various selected substances, data from National Survey of Drug Use and Health 


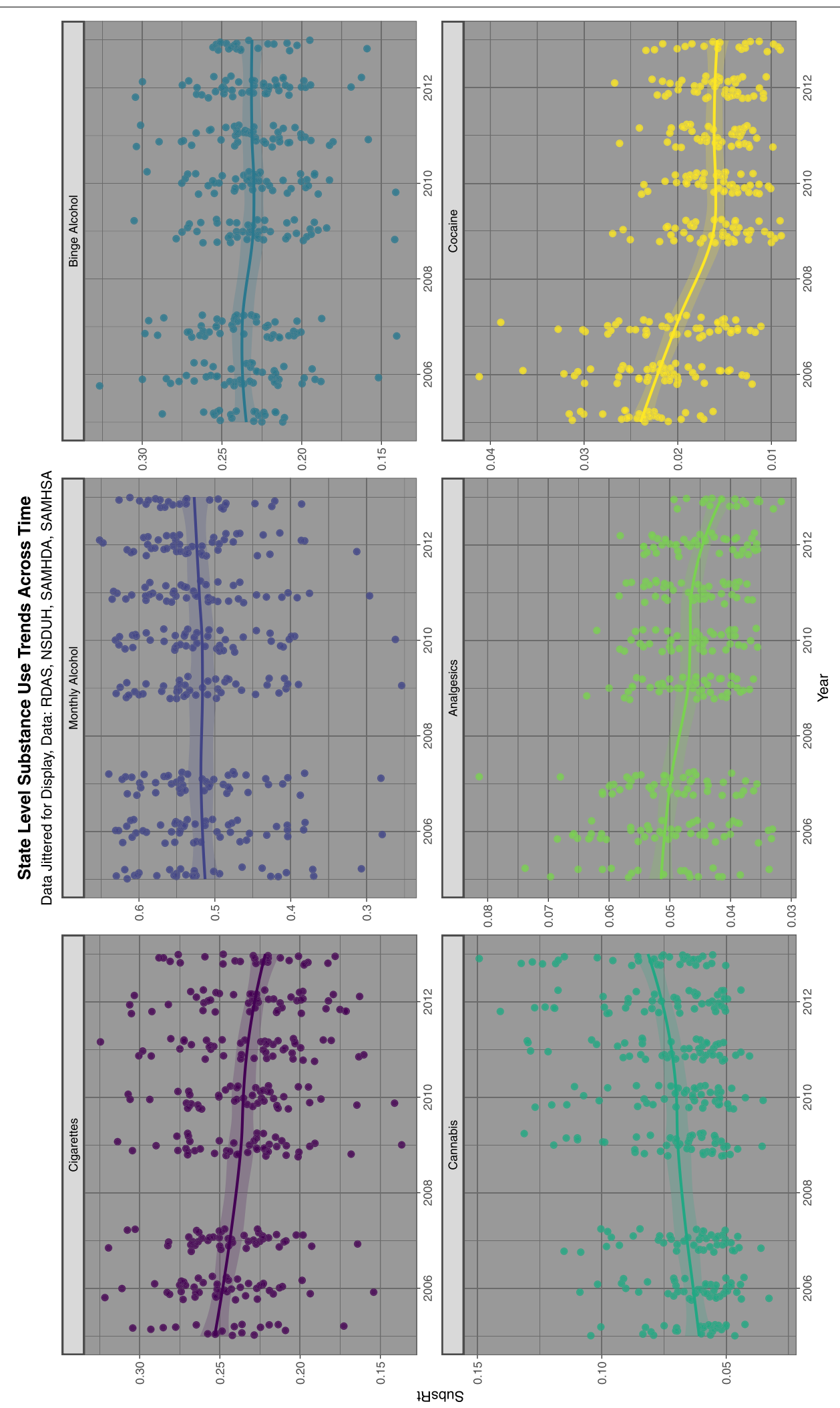

Fig. 3 Trends over time of various selected estimates of cannabinoid exposure, data from National Survey of Drug Use and Health and Drug Enforcement Agency 

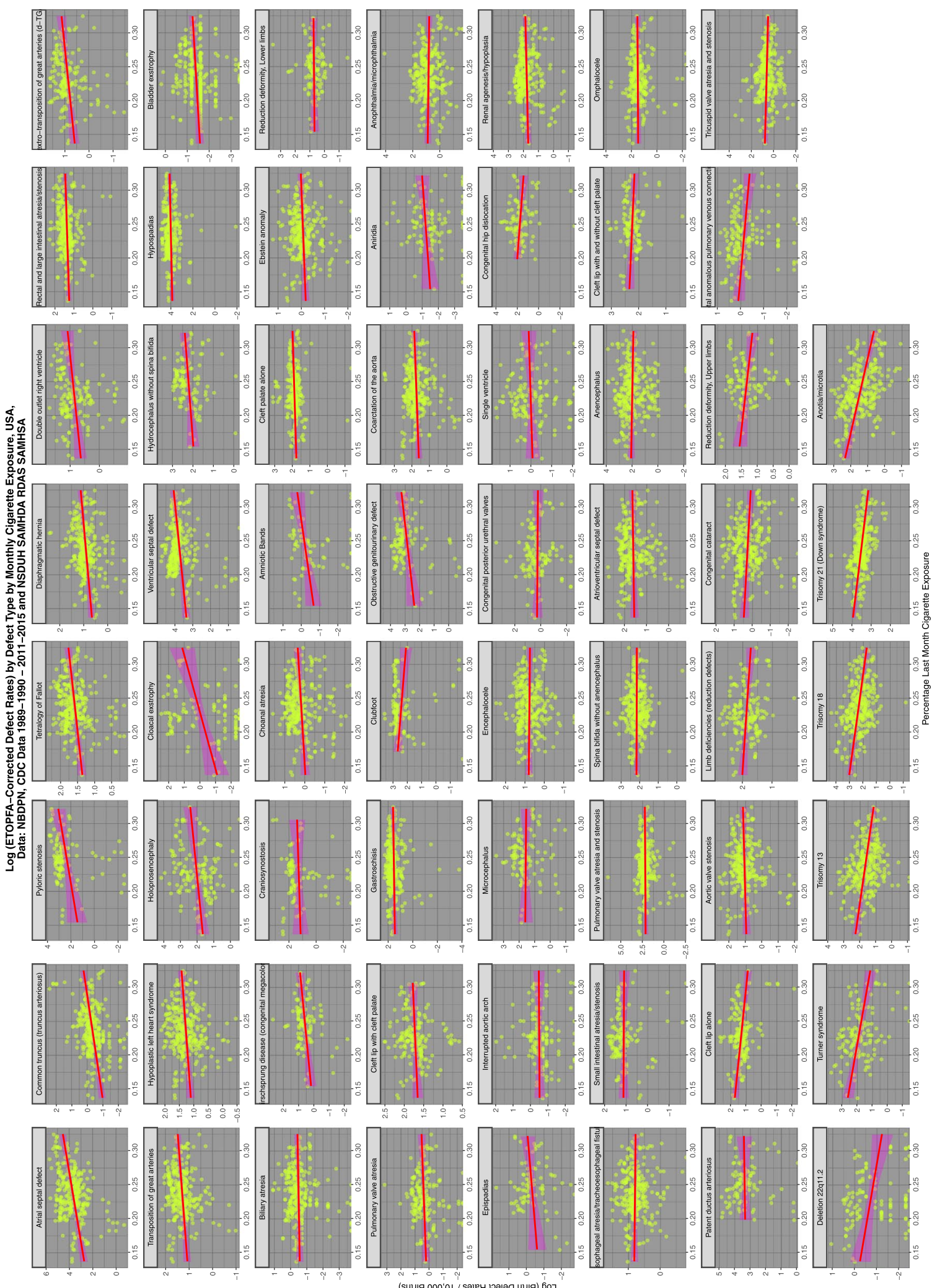

Fig. 4 Trends of various congenital anomaly incidence rates in relationship to tobacco exposure 
Supplementary Fig. 2 shows the relationship of the ETOPFACARs to last month alcohol use. Similar appearances are seen.

Moving to Fig. 5 and considering the relationship of ETOPFACARs to cannabis exposure the pattern changes dramatically from weak associations to many clearly strongly positive and apparently highly significant associations.

Figure 6 shows the relationship of the ETOPFACAR to THC exposure. Many of these relationships are clearly positive and highly significant.

Figure 7 shows the relationship of the ETOPFACARs to state level estimated cannabidiol exposure. Some relationships appear to be positive, particularly in the top line of CAs.

Supplementary Table 3 provides details of the slopes of the ETOPFACARs over time. The table was produced using the purrr-broom package combination in $\mathrm{R}$ using the nest-map-unnest workflow whereby multiple linear models can be processed simultaneously for each $\mathrm{CA}$. The table lists the model $\beta$-estimates, the $\mathrm{t}$-values and various model statistics. Lastly the table lists the point estimates of the E-Values for these regression lines together with the 95\% lower bound of the E-Value.

Table 1 performs a similar function for tobacco exposure. One notes that in this Table 12 ETOPFACARs have minimum E-Values greater than 1.00 .

Supplementary Table 4 performs the same function for binge alcohol exposure. Only two ETOPFACARs have elevated minimum E-Values in this table which are cleft lip alone and epispadias.

Supplementary Table 5 performs the same function for last month alcohol exposure. Here six ETOPFACARs have elevated minimum E-Values.

Contrariwise Table 2, which illustrates the relationship of the ETOPFACARs with cannabis exposure, contrasts sharply with Table 5. In Table 6 one notes that 35 ETOPFACARs are shown to have elevated minimum E-Values. These pertain particularly to cardiovascular system (9 anomalies), urinary tract (6 anomalies), gastrointestinal tract (five anomalies), all five chromosomal anomalies, four musculoskeletal or limb development anomalies (club foot, congenital hip dislocation, limb reduction deficiencies and leg reduction deficiencies), two anomalies each of face and body wall, and one anomaly of brain development.

Supplementary Table 6 performs the same function for estimated THC exposure. In this Table 40 ETOPFACARs have minimum E-Values greater than 1.00. Chromosomal and cardiovascular defects are particularly featured but microtia, limb and leg reduction defects, club foot, gastroschisis, omphalocele, anencephalus, spina bifida, esophageal atresia, small and large intestinal stenosis or atresia and obstructive genitourinary defects and congenital posterior urethral valves also feature.

As shown in Table 3 the list of ETOPFACARs with minimum E-Values greater than 1.00 is shorter for cannabidiol. Eleven defects are featured which are in order: congenital dislocation of the hip, small intestinal stenosis or atresia, biliary atresia, obstructive genitourinary defect, large bowel atresia or stenosis, Hirschsprungs disease (congenital megacolon), esophageal atresia, diaphragmatic hernia cleft palate, reduction deformities of the legs and transposition of the great vessels.

Hence from this series of data we note that the sequence of teratogens is THC $(40$ CAs $)>$ cannabis $\quad(35$ CAs $)>$ tobacco $\quad(11 \quad$ CAs $)>$ cannabidiol $\quad(11$ CAs) $>$ monthly alcohol (5 CAs) $>$ binge alcohol (2 CAs).

To aid with understanding and comparison these minimum E-Values are also presented graphically using a log scale. A horizontal line marks the literature described cut-off for causality at $(\log ) 1.25$ [67]. Supplementary Fig. 3 shows the minimum E-Values for ETOPFACARs over time.

Figure 8 lists the E-Values by CA for those ETOPFACARs which reported elevated finite minimum E-Values for tobacco.

Supplementary Fig. 4 and Figs. 9, 10, 11, 12 do this for binge alcohol, last month alcohol, cannabis, THC and cannabidiol exposure respectively. One notes that the graph for THC clearly has more defects listed.

\section{Categorical exposure survey}

Exposure data was categorized to allow the calculation of key parameters of public health interest such as the prevalence ratio, the aetiological fraction in the exposed and the population attributable risk.

In the following categorical analysis the data was taken from the raw unadjusted NBDPN rates themselves i.e. ETOPFACARs were not used in this series.

Figure 13 shows boxplots by $\mathrm{CA}$ and contrasts the highest and lowest quintiles of cigarette exposure by $\mathrm{CA}$ listing them in the order of the decreasing ratios between the highest and lowest quintiles.

Supplementary Figs. 5, 6, 7, 8 and Figs. 14 and 15 do this for binge alcohol, last month alcohol, analgesic, cocaine, last month cannabis and cannabidiol exposure. Cannabidiol quintiles in Fig. 15 are not grouped by year but calculated across the whole period.

Supplementary Table 7 presents the numbers born with and without CAs in the highest and lowest quintiles of tobacco use states. The Prevalence Ratio (like the Odds Ratio for cohort studies), Attributable Fraction in the Exposed (AFE), the Population Attributable Risk (PAR), the Chi Squared value and the P-level of significance is also 

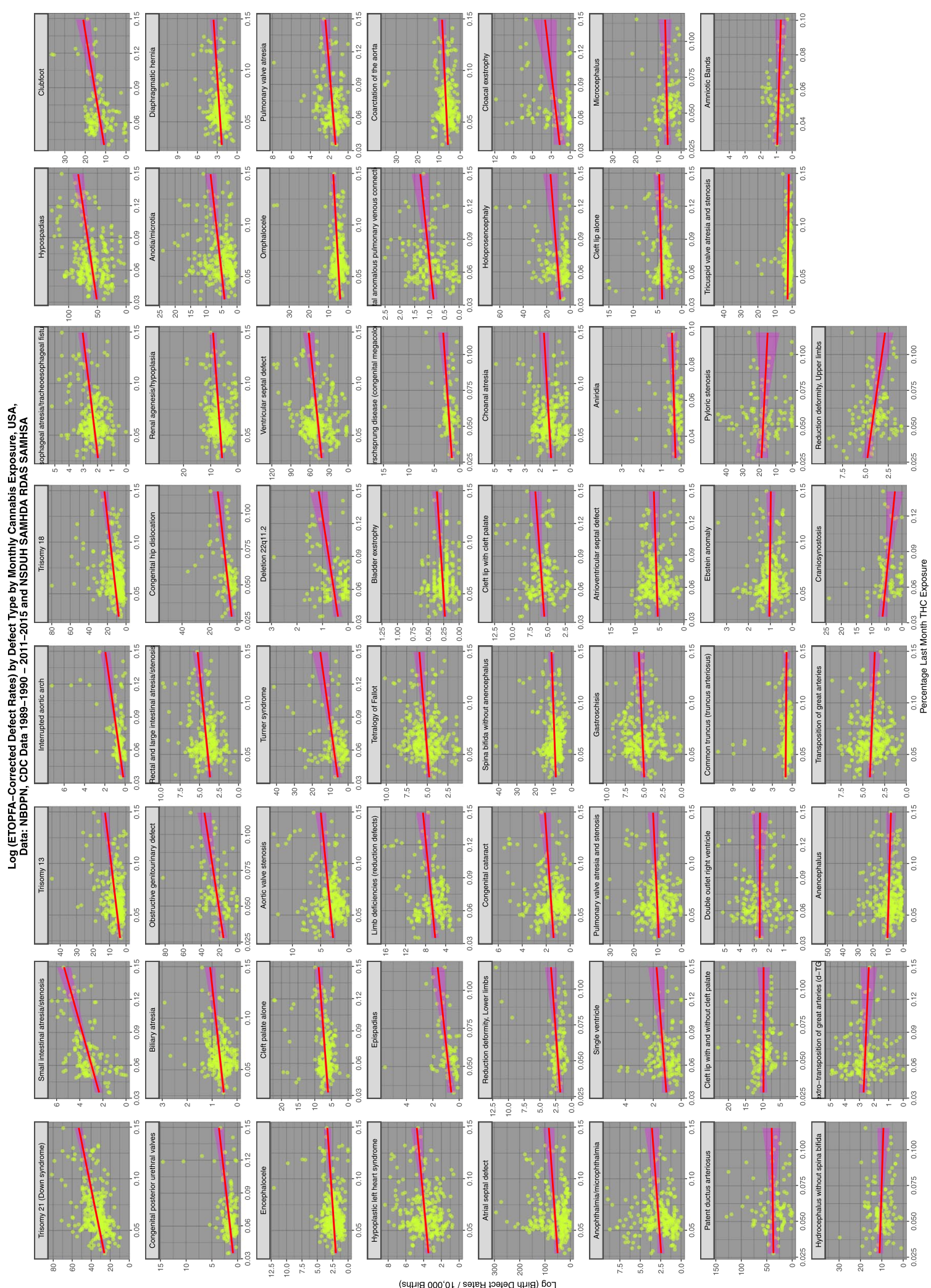

Fig. 5 Trends of various congenital anomaly incidence rates in relationship to cannabis exposure 

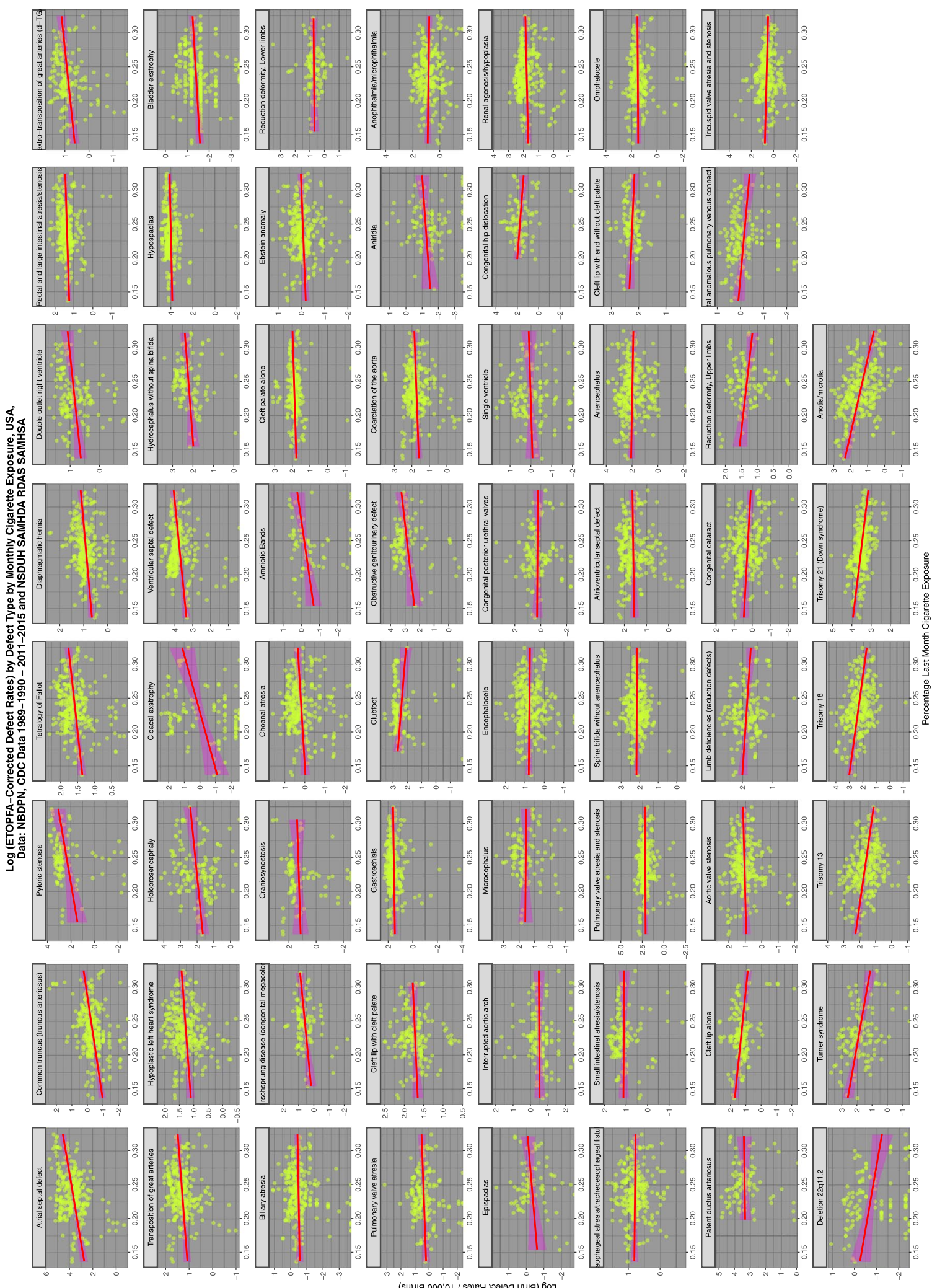

Fig. 6 Trends of various congenital anomaly incidence rates in relationship to THC exposure 

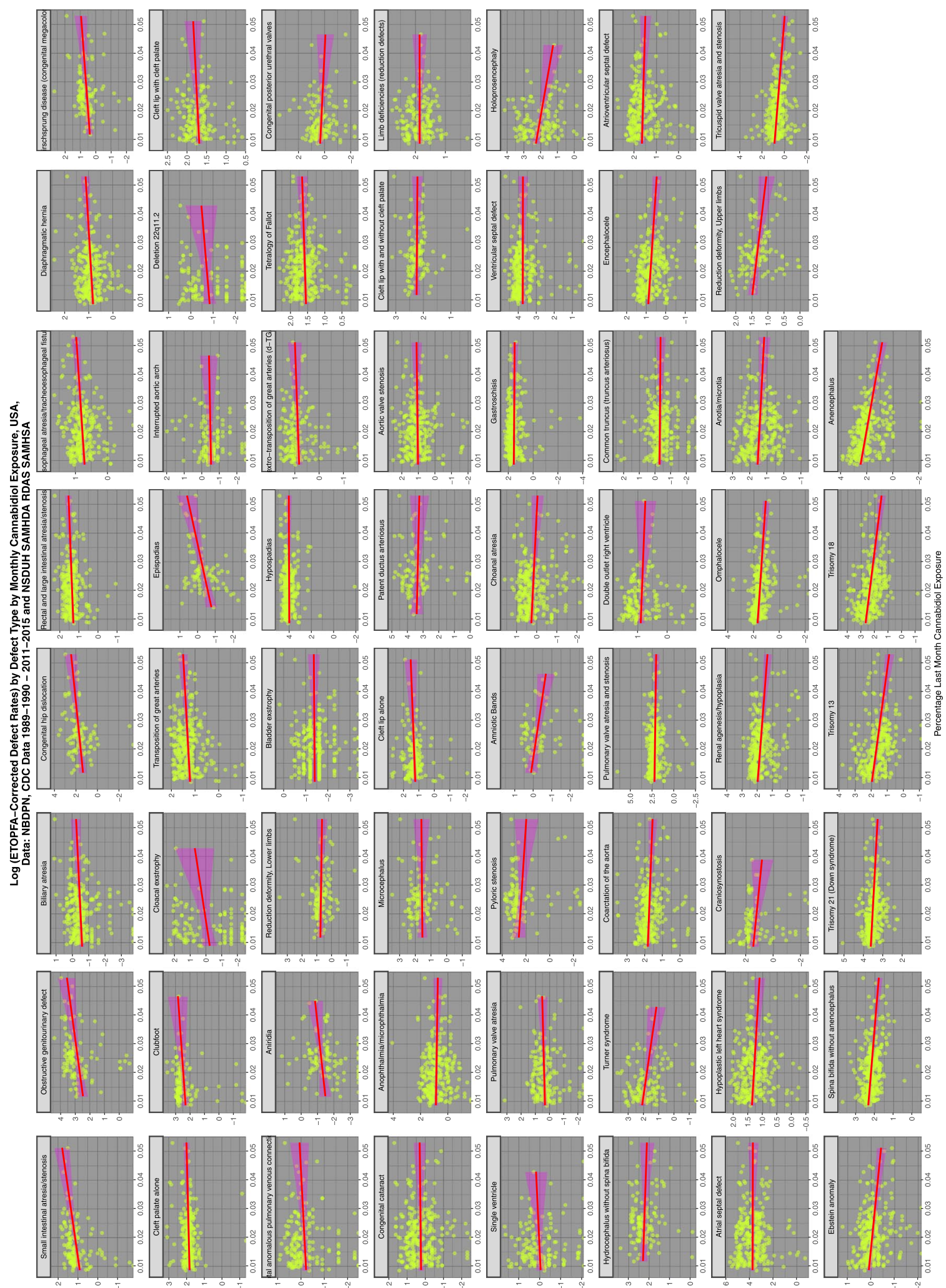

Fig. 7 Trends of various congenital anomaly incidence rates in relationship to cannabidiol exposure 


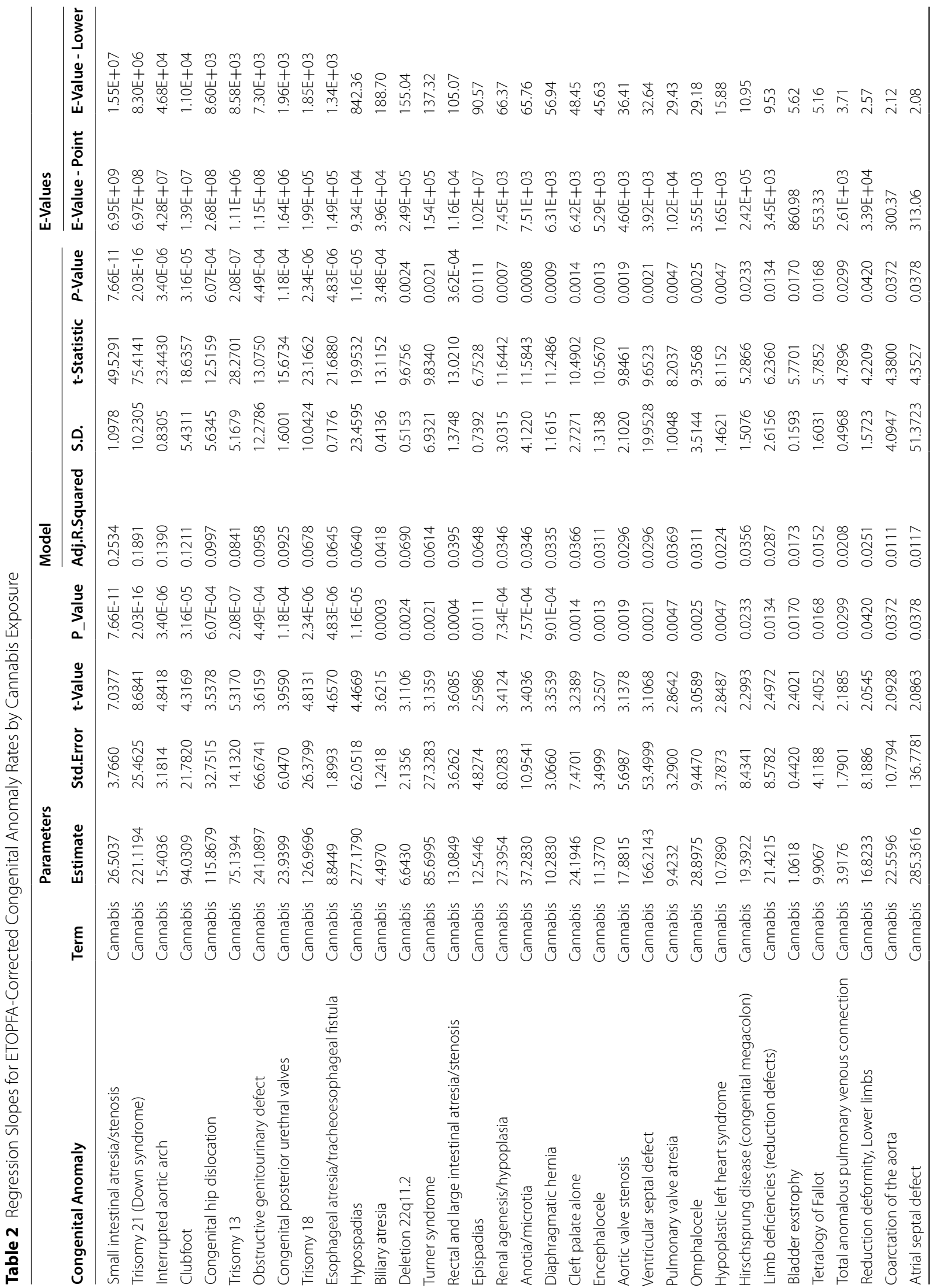




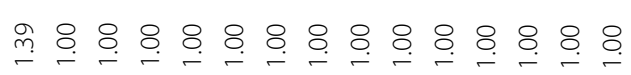

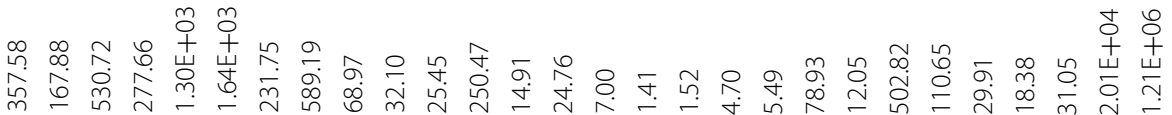

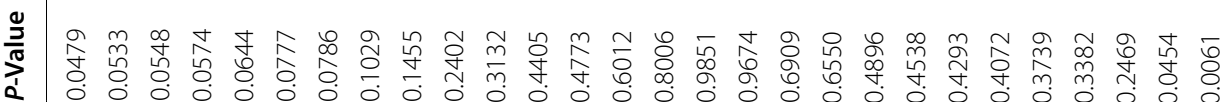

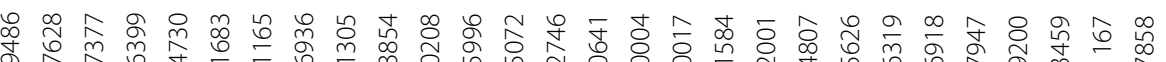

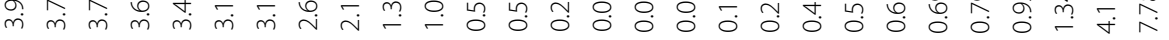

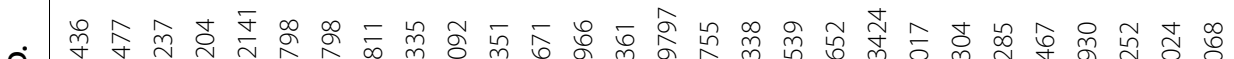

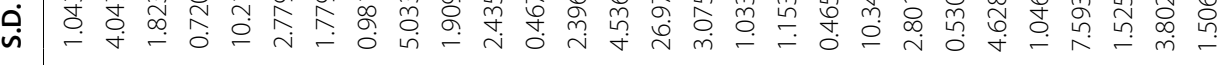

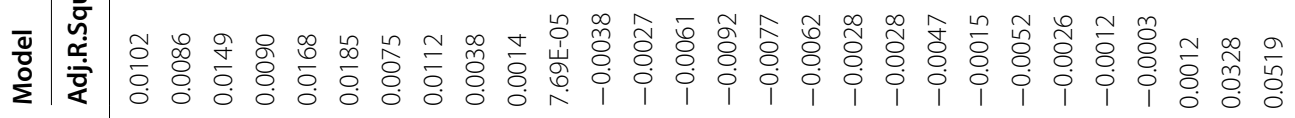

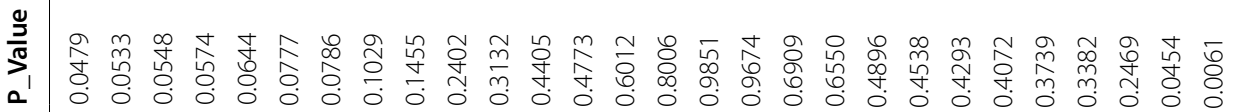

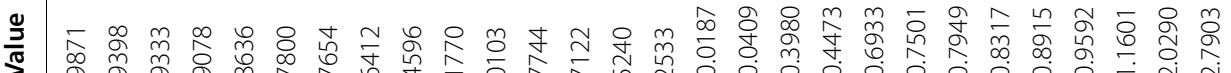

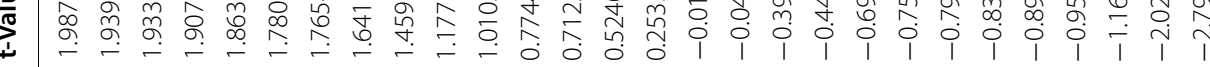

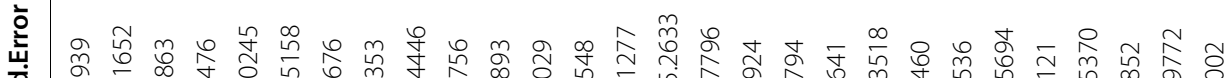

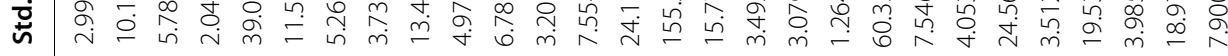

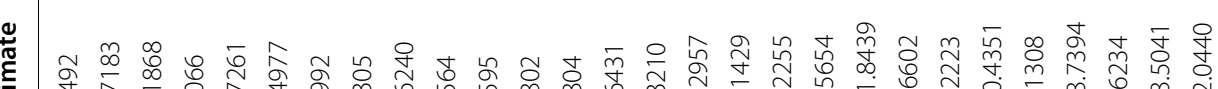

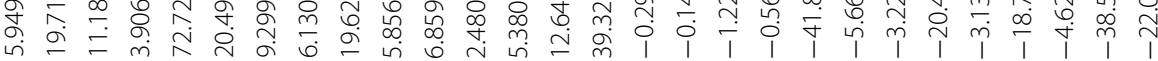

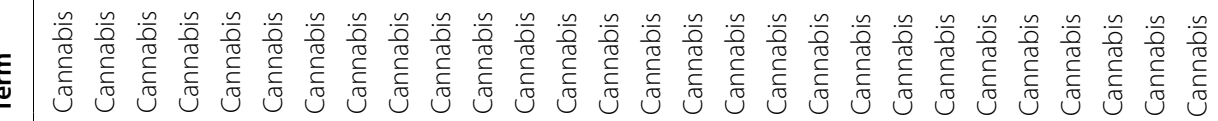

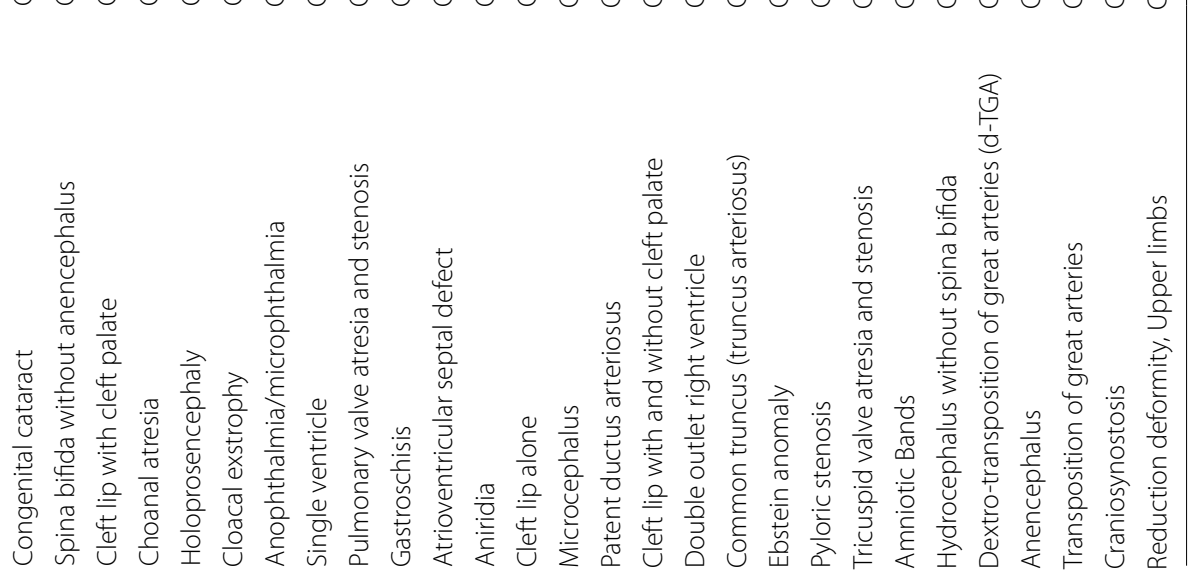




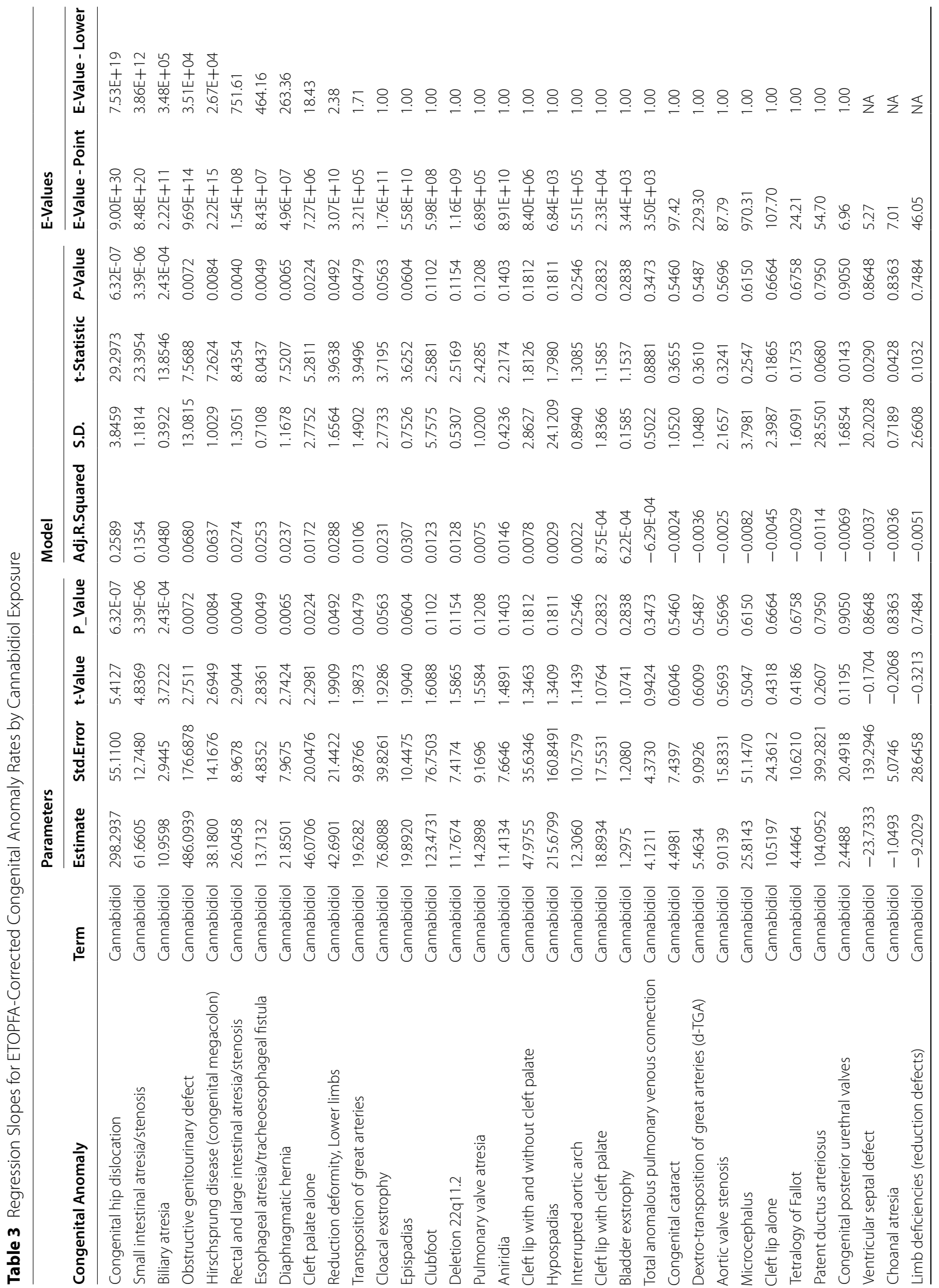




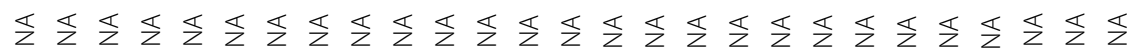

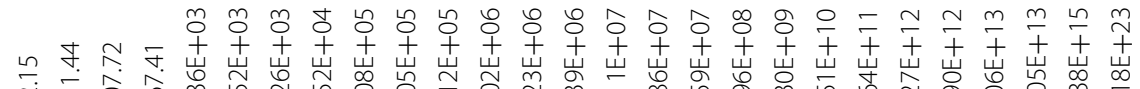

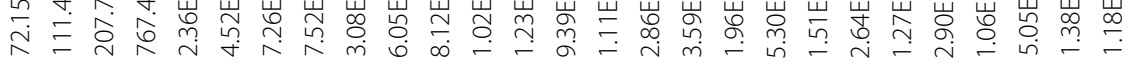

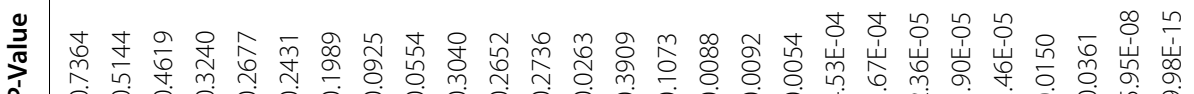

m

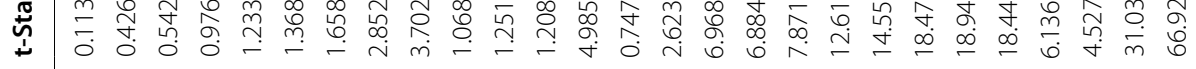

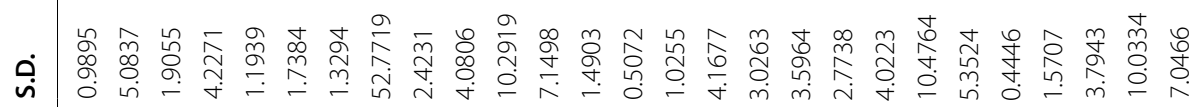

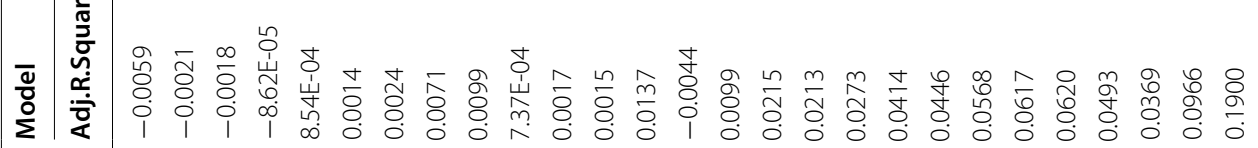

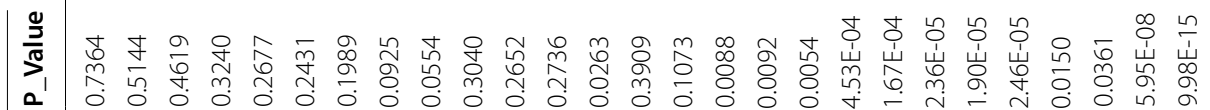

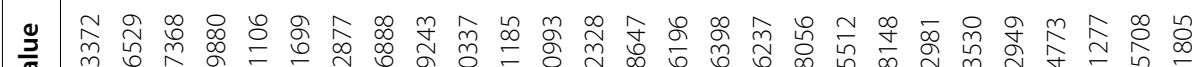

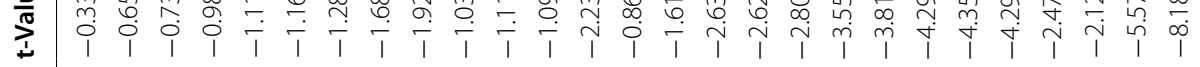

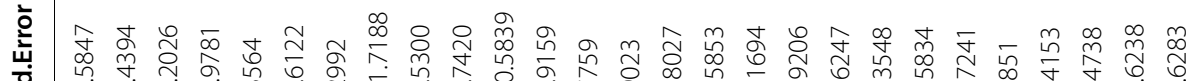

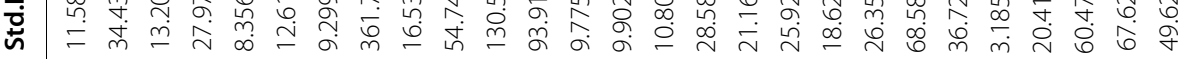

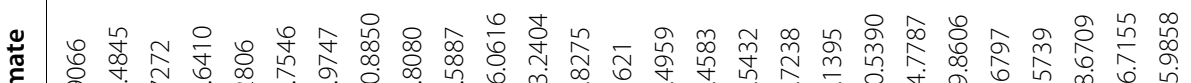

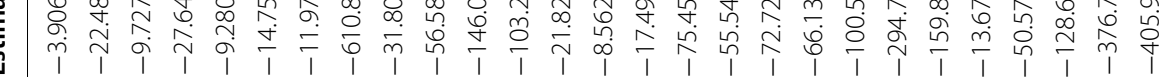

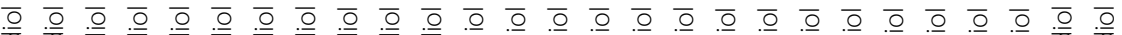

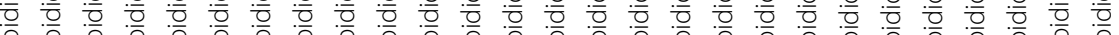

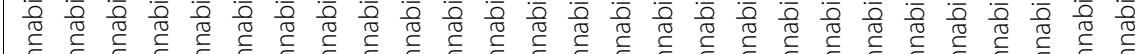

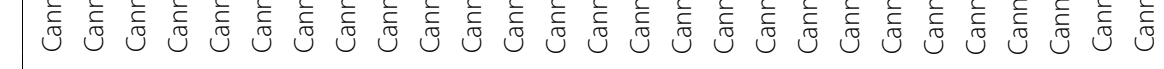




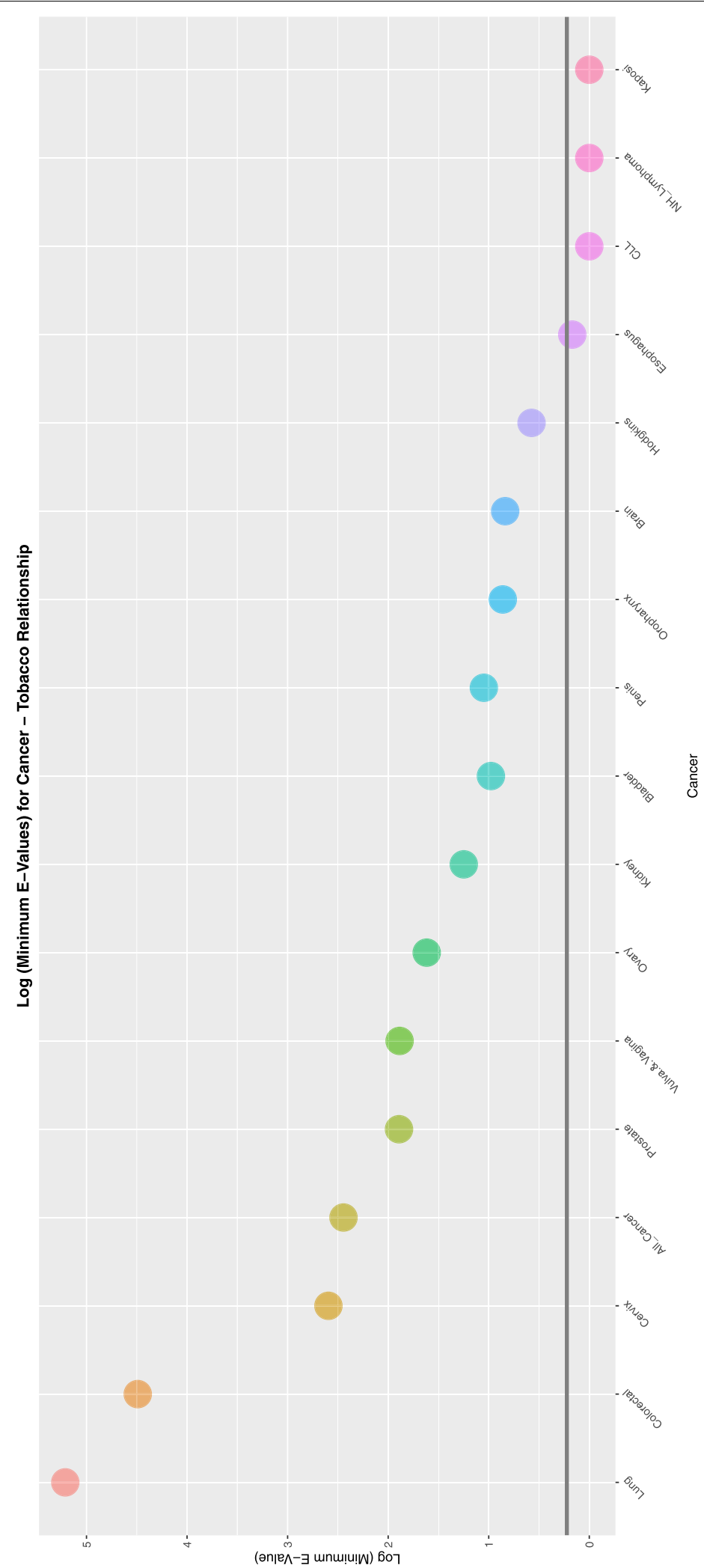

Fig. 8 E-Values of regression lines of relationship of congenital anomaly incidence rates with tobacco exposure 


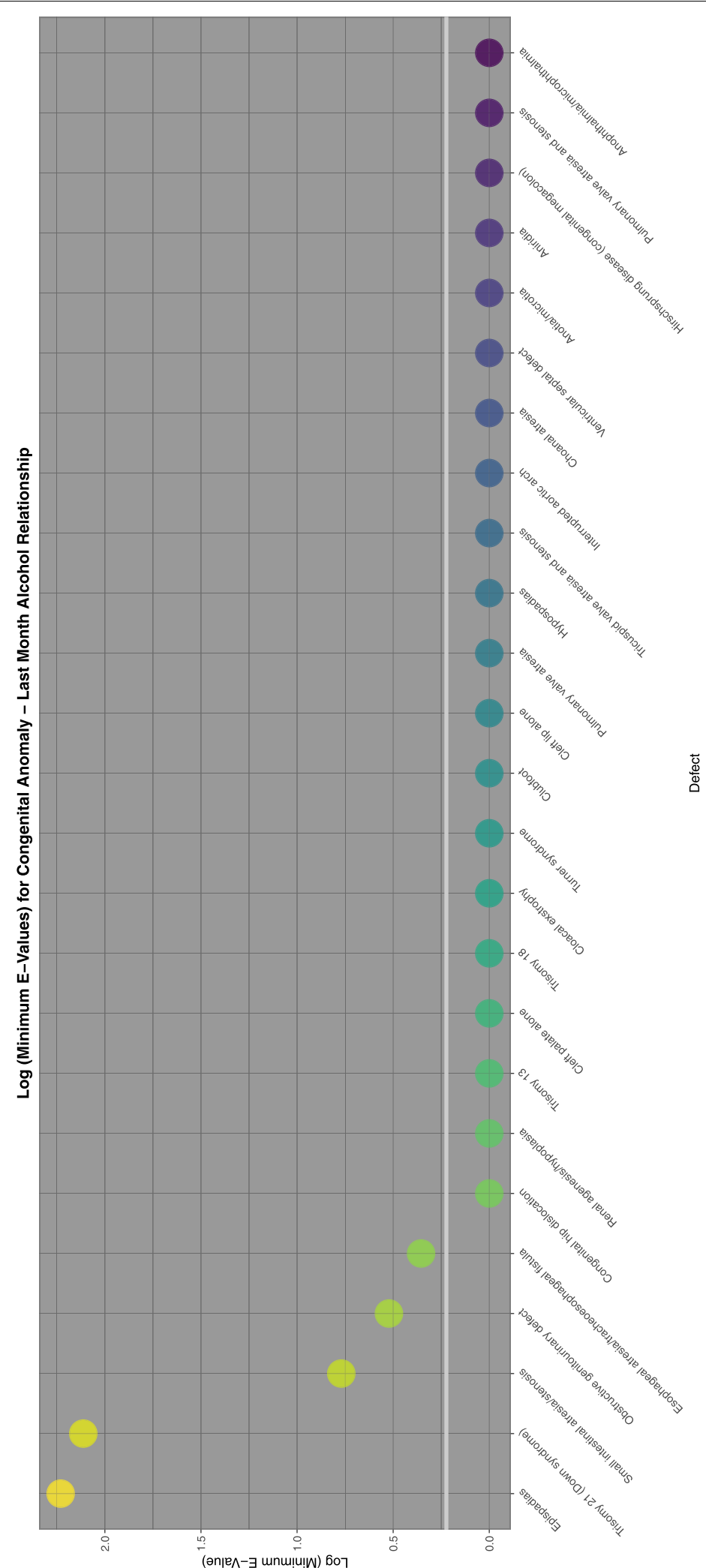

Fig. 9 E-Values of regression lines of relationship of congenital anomaly incidence rates with last month exposure 


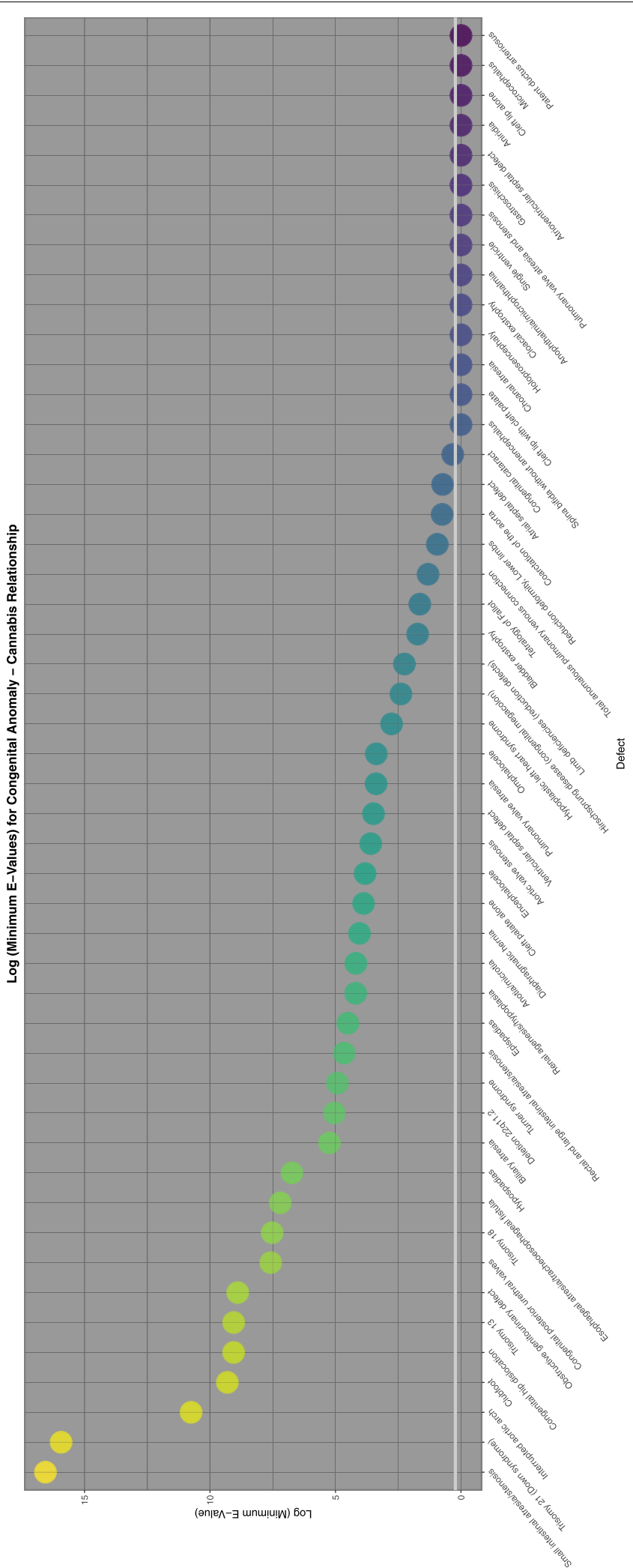

Fig. 10 E-Values of regression lines of relationship of congenital anomaly incidence rates with cannabis exposure 


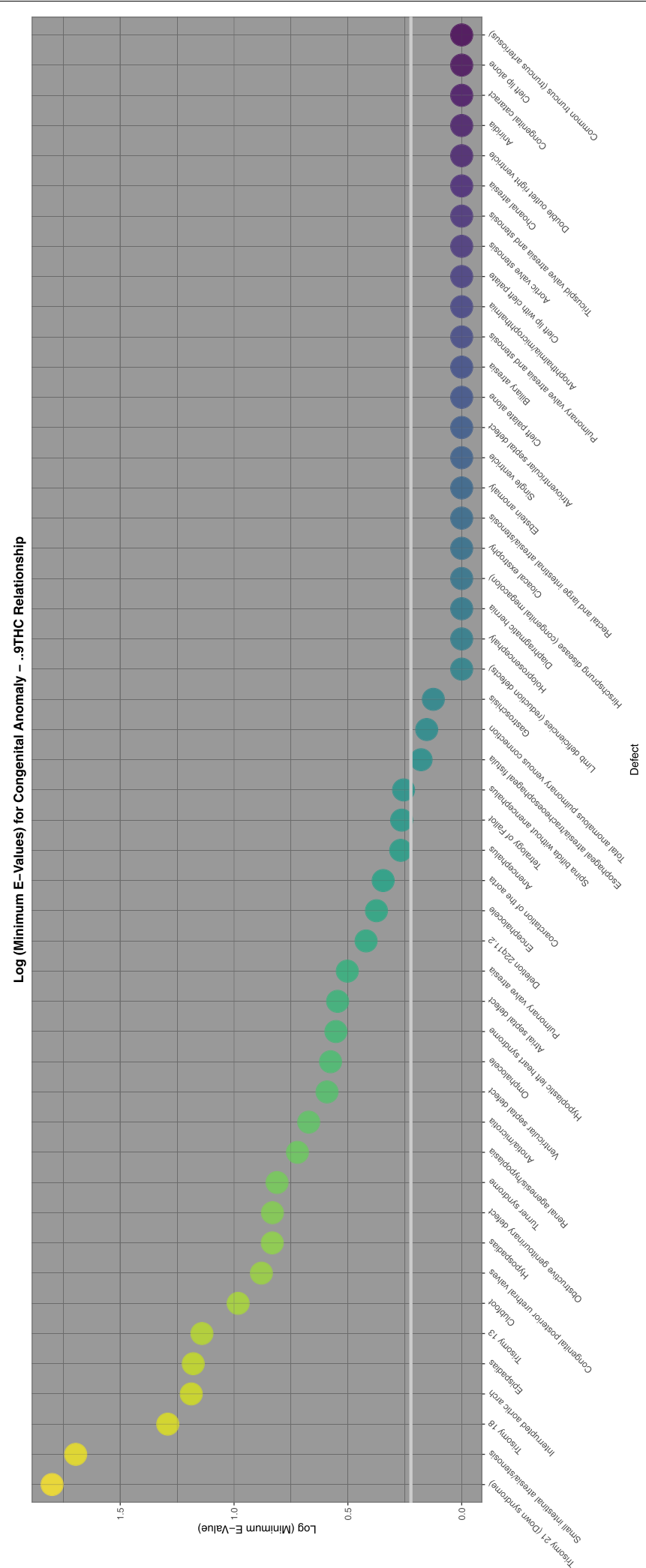

Fig. 11 E-Values of regression lines of relationship of congenital anomaly incidence rates with THC exposure 


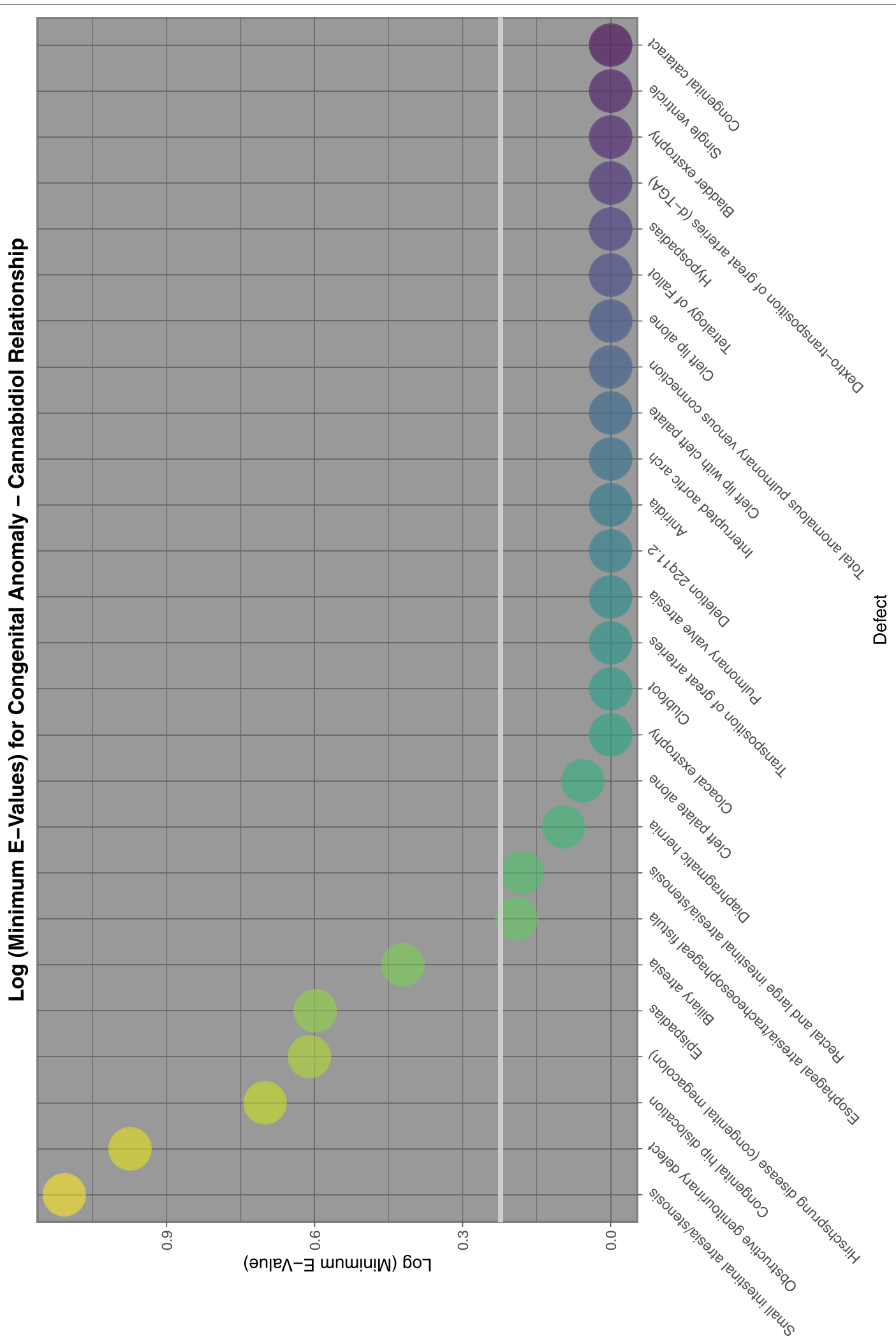

Fig. 12 E-Values of regression lines of relationship of congenital anomaly incidence rates with cannabidiol exposure 

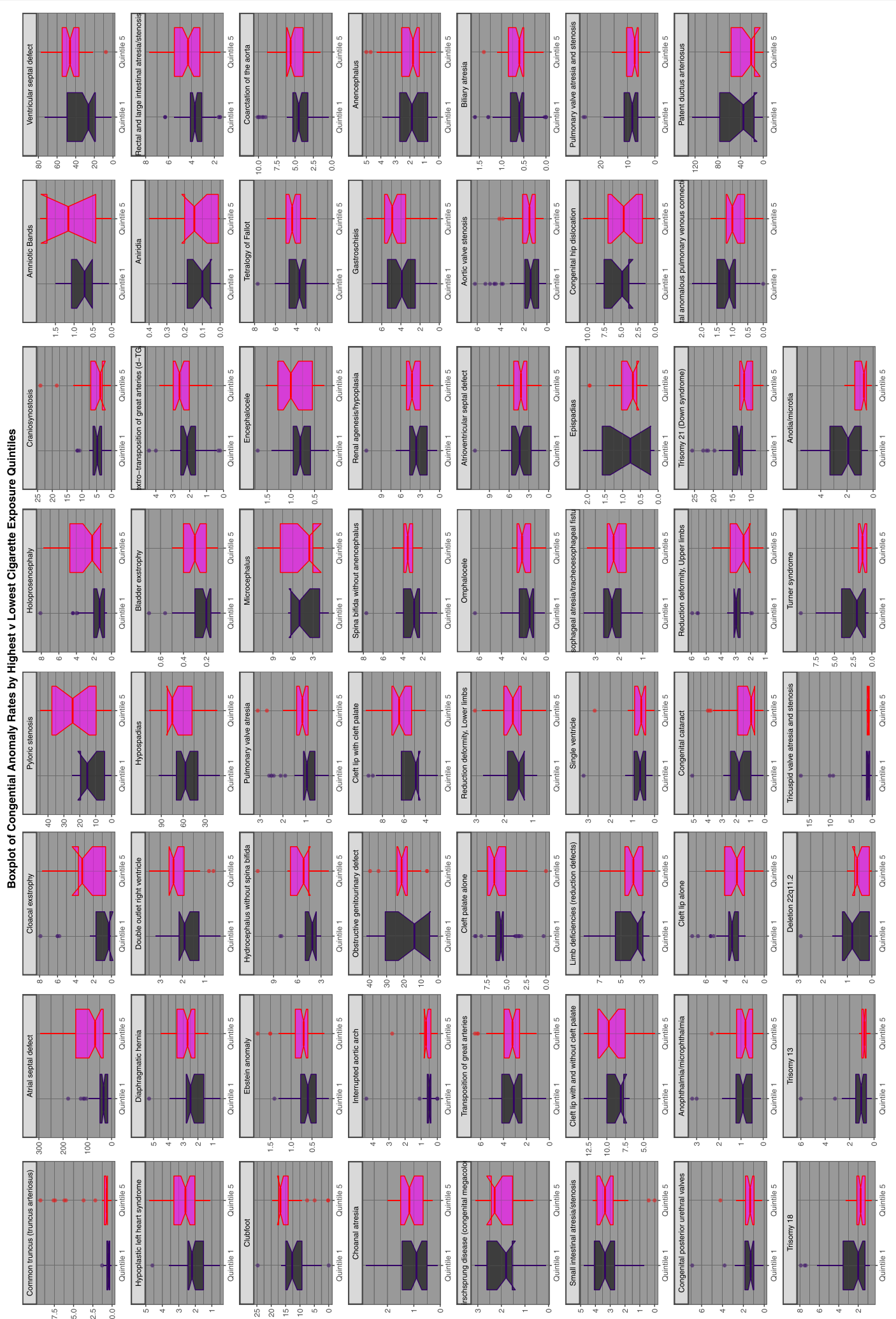

Fig. 13 Categorical analysis of congenital anomaly incidence rates rates for extreme quintiles of tobacco exposure 


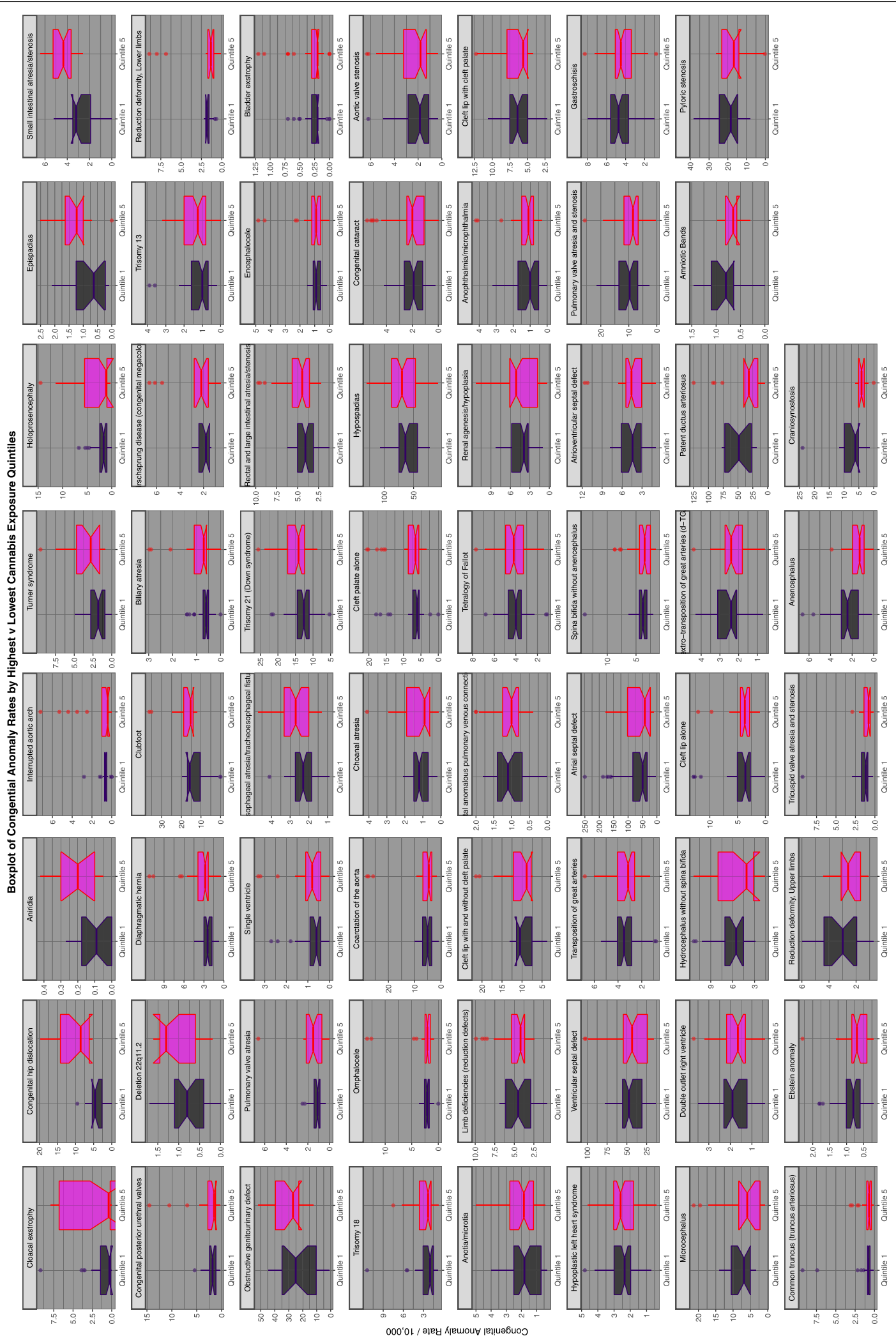

Fig. 14 Categorical analysis of congenital anomaly incidence rates for extreme quintiles of last month cannabis exposure 

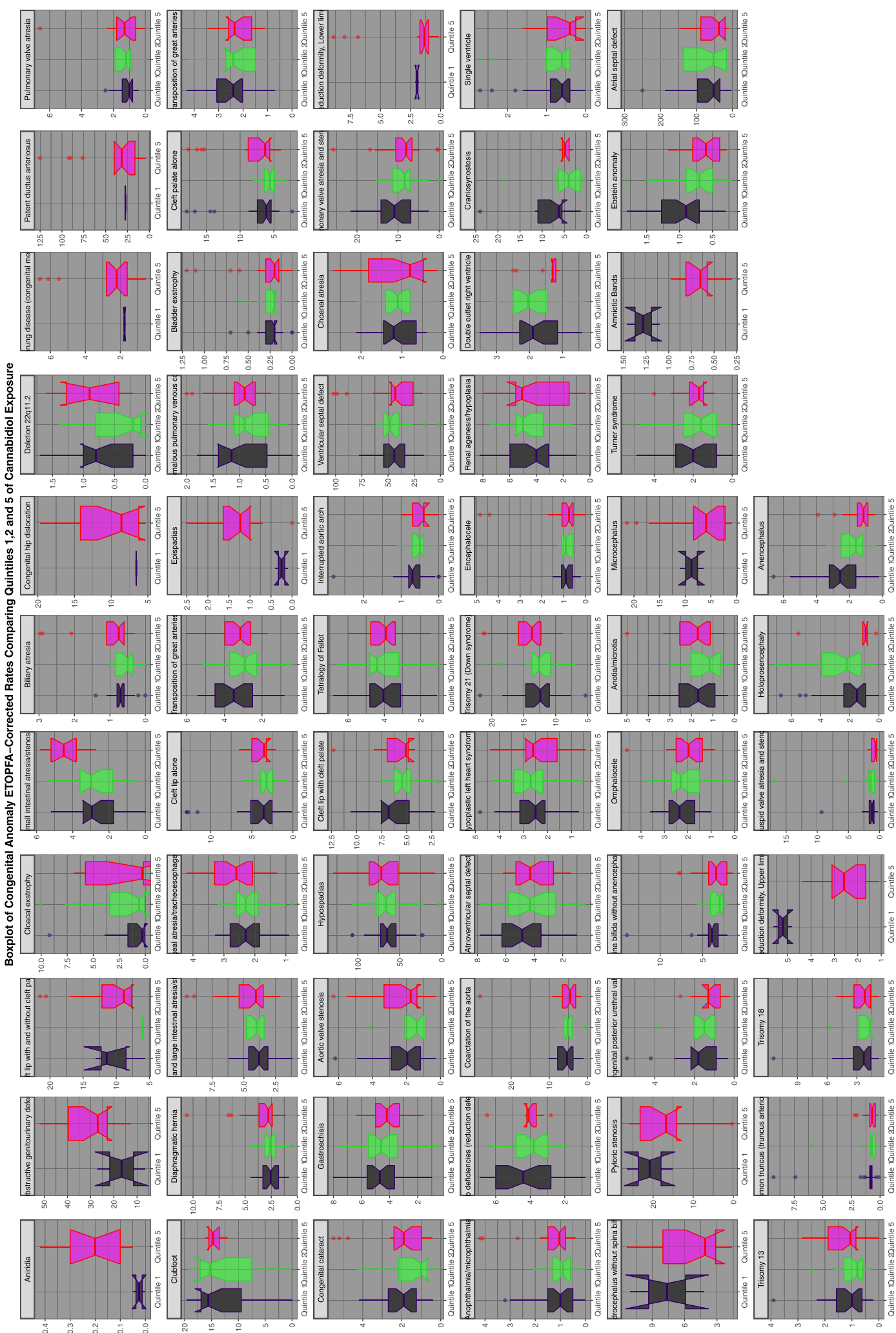

Fig. 15 Categorical analysis of congenital anomaly incidence rates for extreme quintiles of canabidiol exposure 
shown. The right most columns show the point estimate for the E-Value together with its 95\% lower bound. In this Supplementary Table 7 defects are noted to have minimum E-Values elevated above 1.00.

Supplementary Tables 8, 9, 10 and Tables 4, 5 perform a similar function for binge alcohol, analgesics, cocaine, cannabis and cannabidiol respectively. As the CAs tracked by NBDPN / CDC changed over time as the cannabidiol exposure was falling 11 defects have no entries in Quintile 1 (see Fig. 15 for details). Numbers exposed in Quintile 2 were used for these CAs. In these five tables one notes respectively that 1, 21, 27, 10 and 11 CAs demonstrate elevated minimum E-Values. These data suggest that cannabis (21 defects) is the third most important teratogen behind analgesics (27 CAs) and tobacco (26 CAs). Teratogenesis from cannabidiol also appears to be significant (11 CAs).

As shown in Table 4 six cardiovascular anomalies, five chromosomal, five gastrointestinal, two urinary, two limb, and one each facial (Holoprosencephaly), body wall (Diaphragmatic hernia) and CNS (spina bifida without anencephalus) anomaly are accompanied by higher E-Values in the high cannabis use quintiles. Interestingly both congenital posterior urethral vales and diaphragmatic hernia and several gastrointestinal anomalies appear both on this list and on the list of elevated E-Values shown in Table 2 where cannabis exposure is treated as a continuous covariate.

As indicated in Table 512 anomalies including three cardiovascular (pulmonary valve atresia, double outlet right ventricle, single ventricle), three gastrointestinal (small intestinal atresia /stenosis, biliary atresia, cloacal extrophy), two chromosomal (Trisomies 14 and 21) and one each limb (clubfoot), body wall (diaphragmatic hernia), face (cleft lip with and without cleft palate) and genitourinary (obstructive genitourinary defect) anomaly were noted to have elevated minimum E-Values in highest cannabidiol exposure quintiles.

For ease of comparison these Prevalence Ratios are presented together by substance in Table 6 . The prevalence ratios for cannabidiol appear in the right hand column and are listed in descending order.

Table 7 presents the Attributable Fractions in the Exposed (AFEs) in a similar manner. One notes that they descend from a strikingly high rate of $79.38 \%$ for cloacal extrophy after cannabis exposure.

Table 8 performs a similar function for Population Attributable Risk (PAR). Cloacal extrophy again heads the list from a PAR of $56.75 \%$ after cannabis exposure.

Applicable $P$-values are listed together by substance in Table 9. In reading this table it should be noted that $P$ values in $\mathrm{R}$ are only computed down to $2.2 \times 10^{-320}$. Such values in the table may be better understood as zeroes.
Minimum E-Values for these comparisons are shown in Table 10 by substance.

\section{Summary of bivariate analyses}

Given that the above tables present a lot of information it is of interest to distil this information down into more intellectually digestible components.

Supplementary Table 11 extracts the 85 ETOPFACARs which have significant E-Values for the 35 cannabis related CAs, the $40 \mathrm{THC}$ related CAs and the 11 cannabidiol CAs considered as continuous variables. The table is arranged in descending order of the lower bound of the E-Values. 37/85 E-Values are greater than 9.0 which is the E-Value for the tobacco-lung cancer relationship and $84 / 85$ are greater than 1.25 which is the quoted cut-off for causality [68].

Table 11 re-lists the 41 CAs listed in Table 20 and retains only the ETOPFACAR with the highest minimum E-Value. In this Table 28/41 are greater than 9.0 and 40/41 are greater than 1.25. On this list 28 CAs are related to cannabis, 5 to THC and 8 to cannabidiol.

To further condense this material Table 12 lists the organ systems of the various CAs listed in descending order of the percentages of the listed CAs for that organ system. It is noted immediately that the list is headed by chromosomal disorders, but that genitourinary, gastrointestinal, limb defects, body wall defects, cardiovascular anomalies and facial anomalies all have more than $50 \%$ of their listed CAs positively and potentially causally associated with one of the various cannabinoids.

A similar exercise can be performed on the CARs (not corrected for ETOPFAs) treated as categorical variables comparing the highest Quintile (Quintile 5) with the lowest quintile (Quintile 1, or the absence of data, Quintile 2).

Supplementary Table 12 shows selected parameters from this comparison extracted for those 31 CARs with elevated minimum E-Values listed in descending order of E-Values. 21 of these CARs are related to cannabis and 12 are related to cannabidiol.

Table 13 removes the duplicates from these CARs and retains the most significant results leaving 23 CARs, 17 related to cannabis and 6 to cannabidiol.

Table 14 lists these various CARs by body system. The results are qualitatively similar to those presented in Table 12 but less dramatic.

\section{Detailed analyses of specific congenital anomalies}

It is of interest to consider two of these defects in detail by way of example of the kinds of space-time analyses 


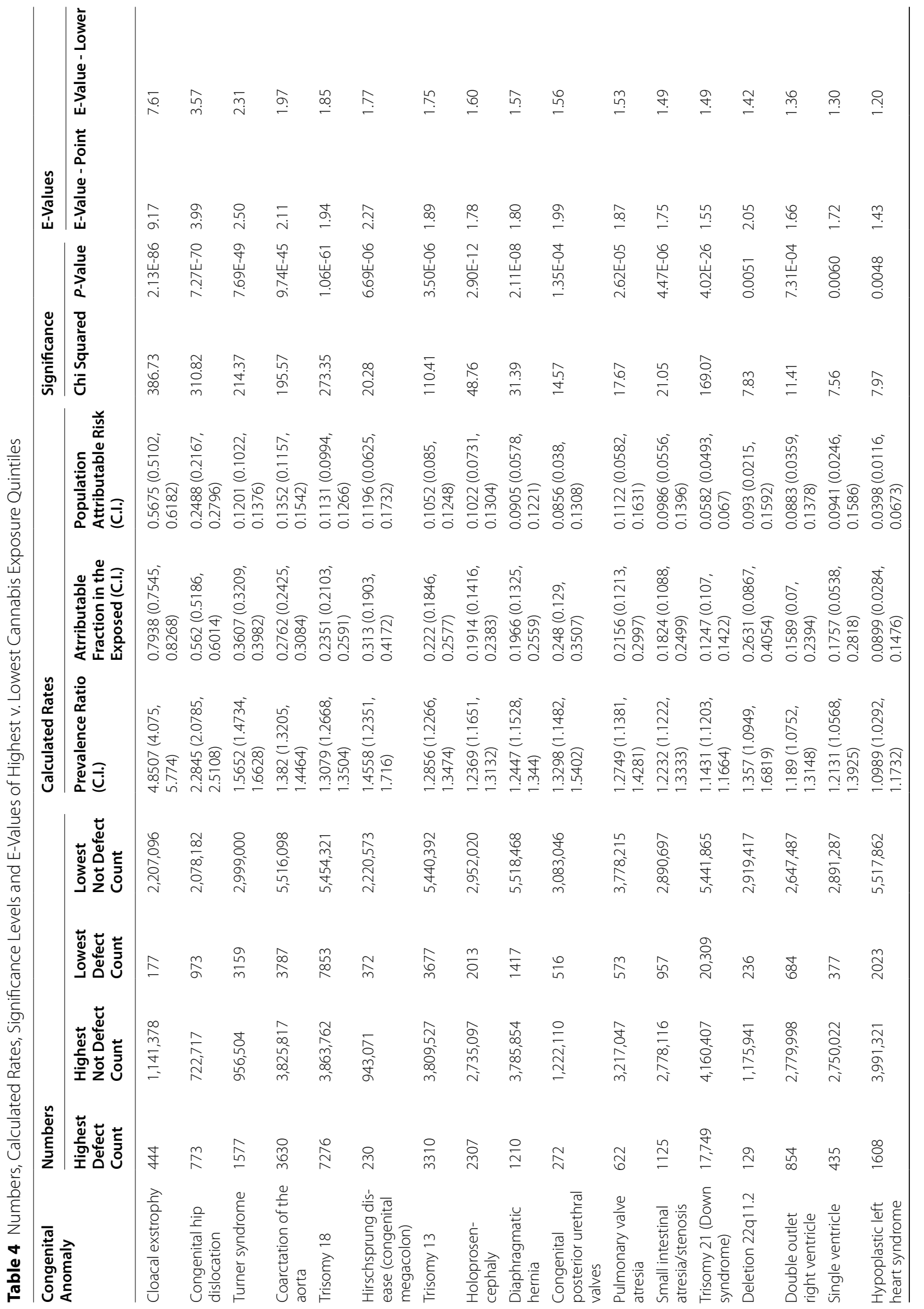




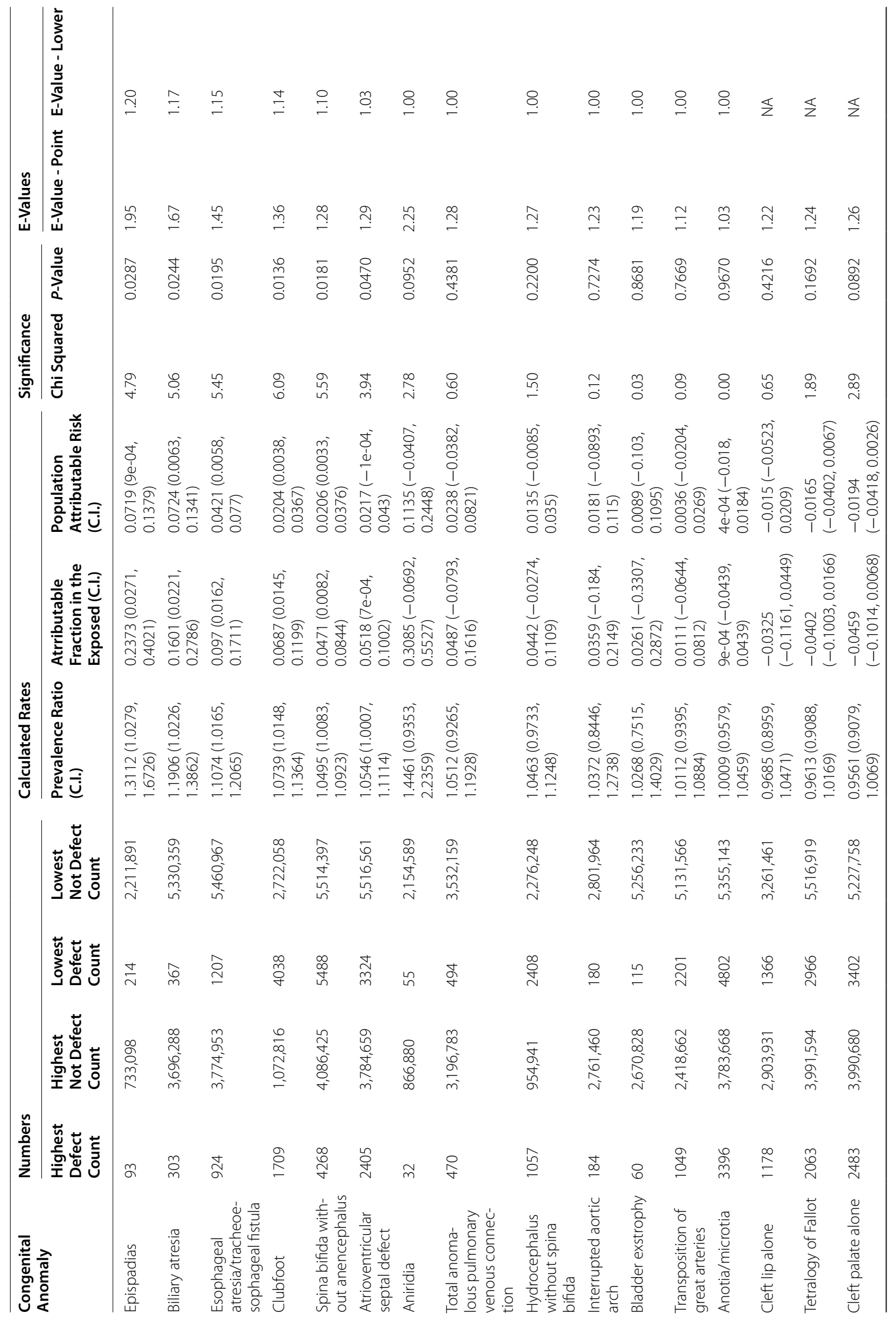




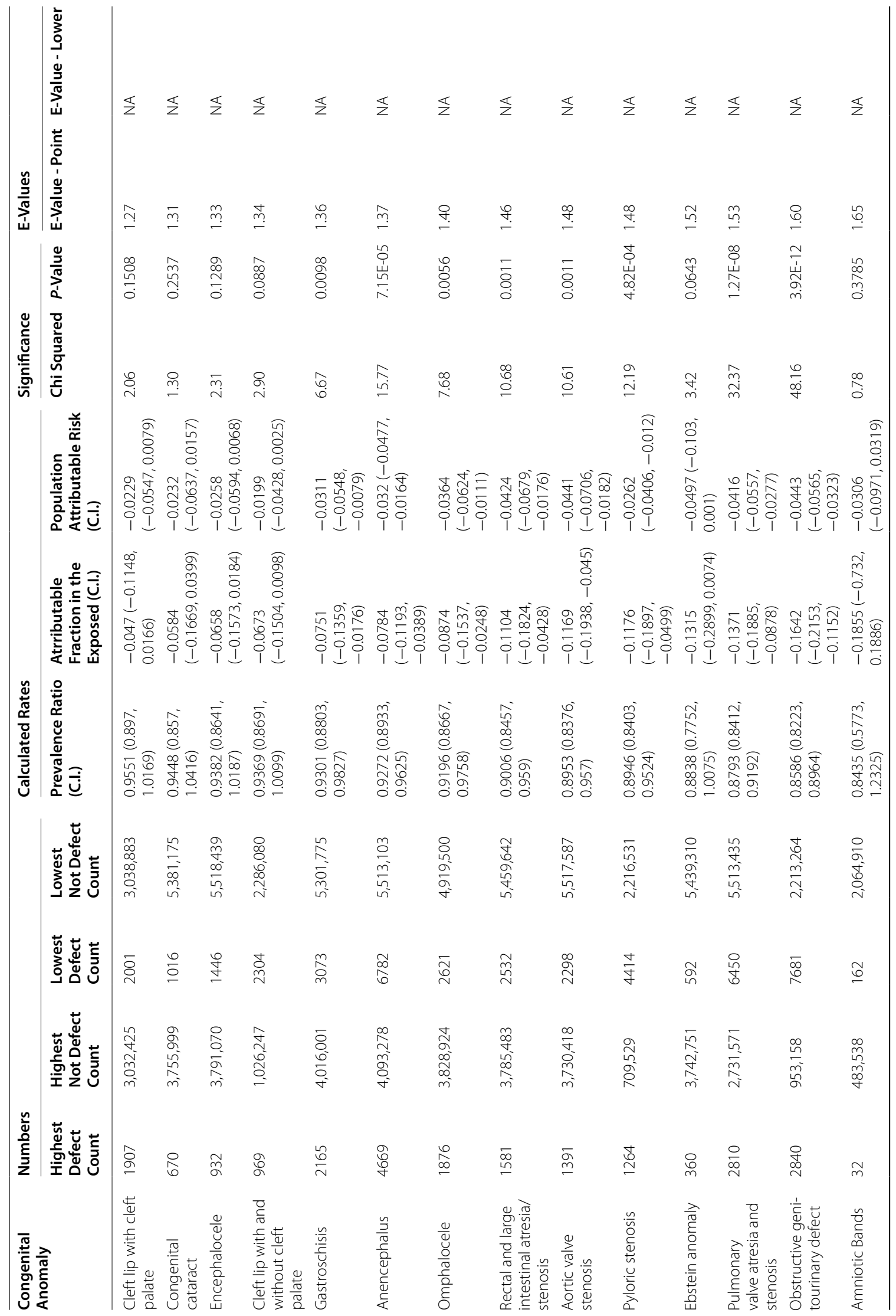




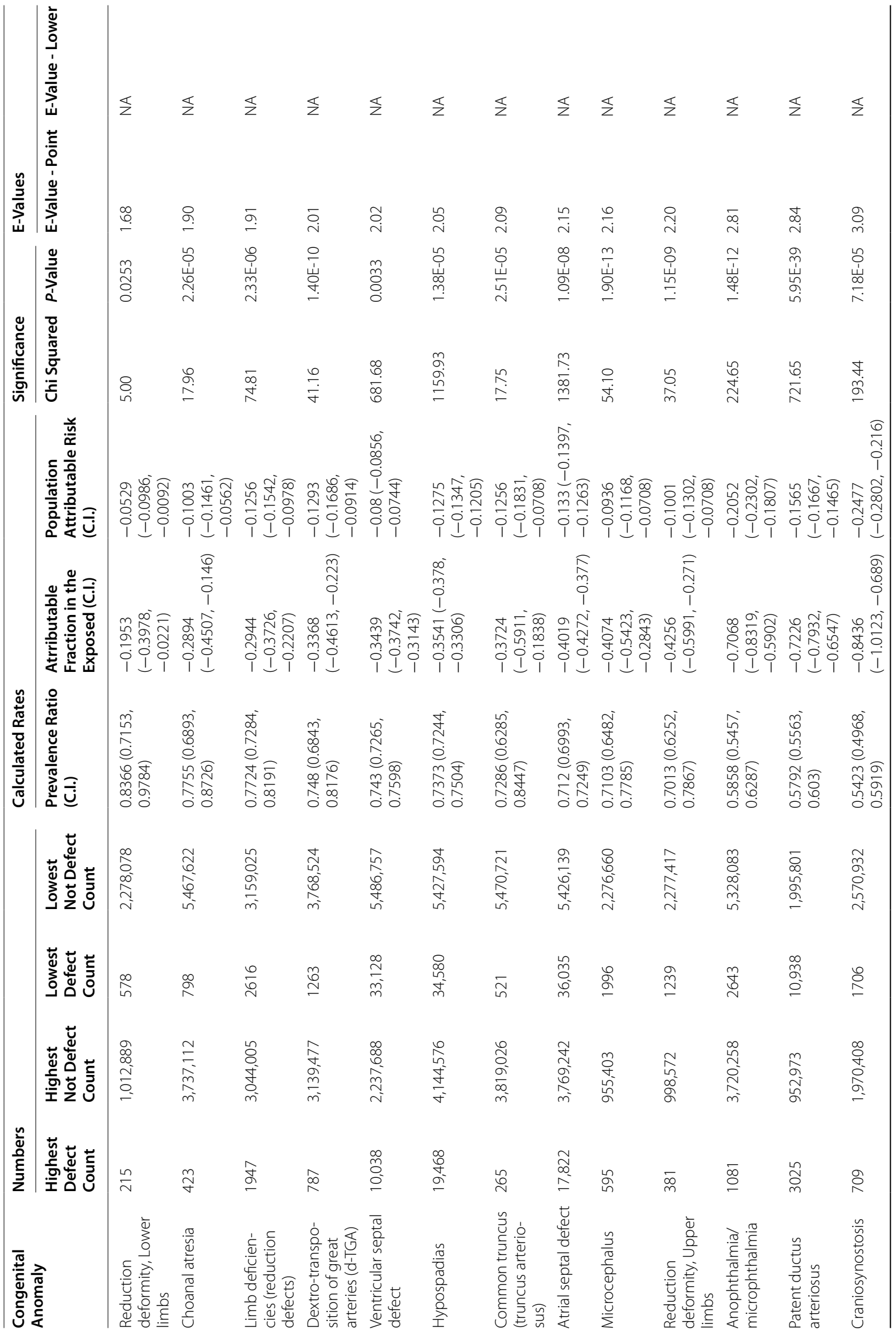




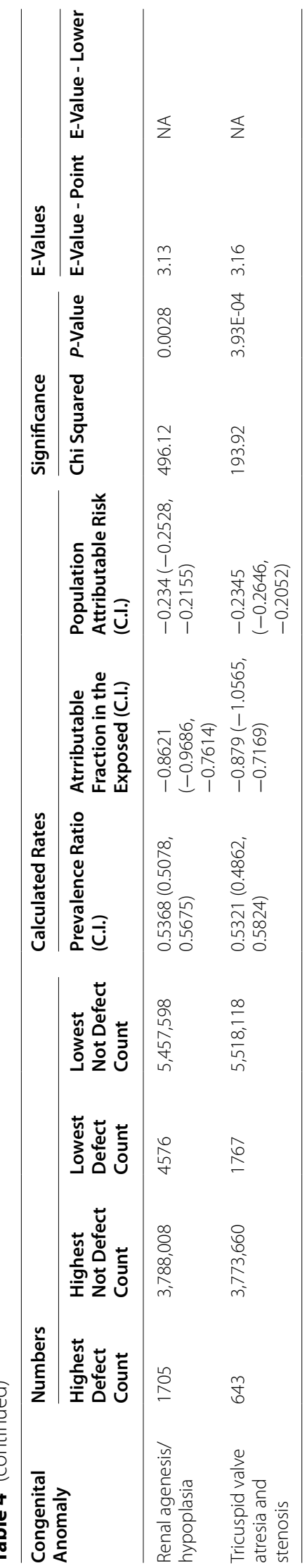




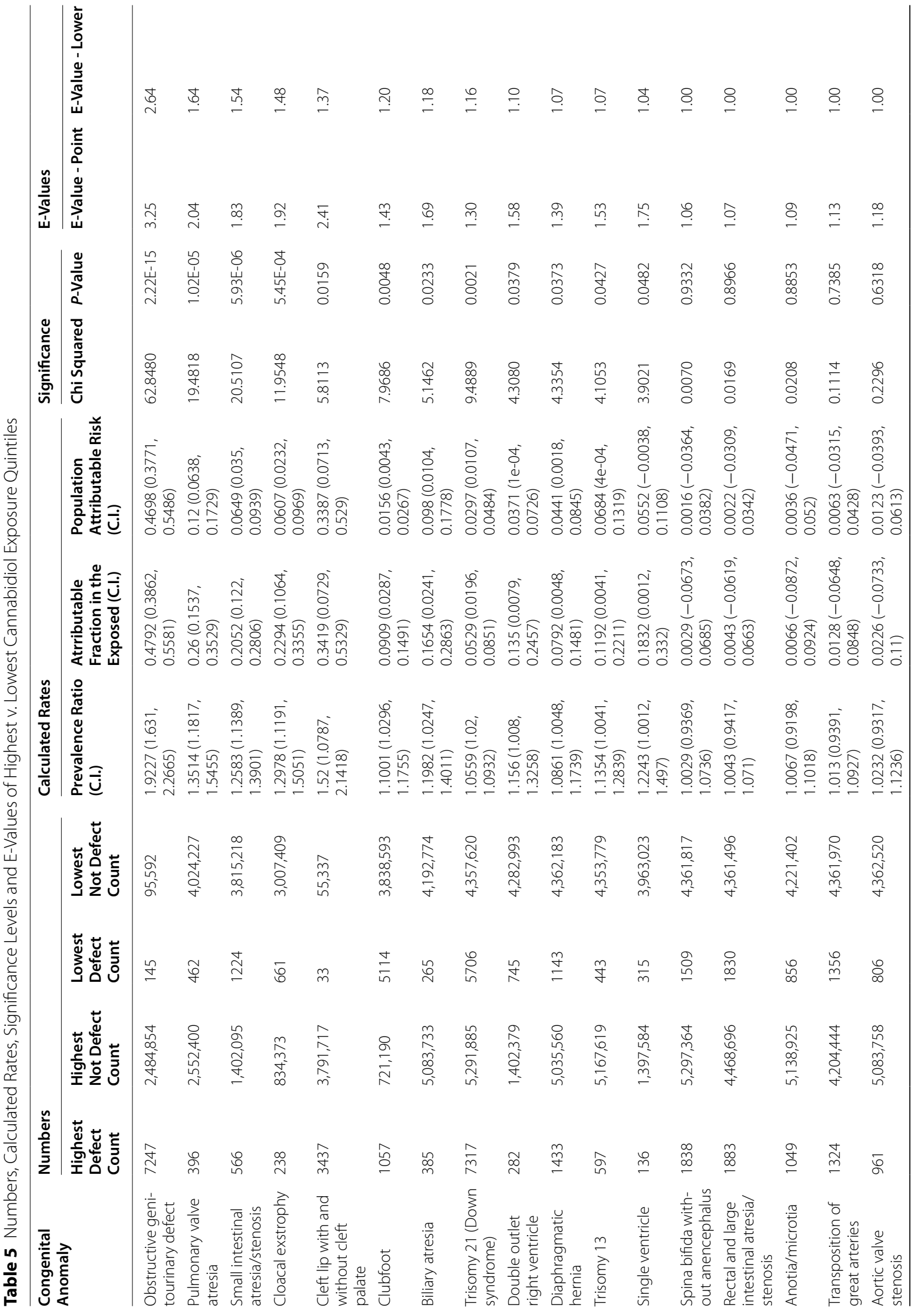




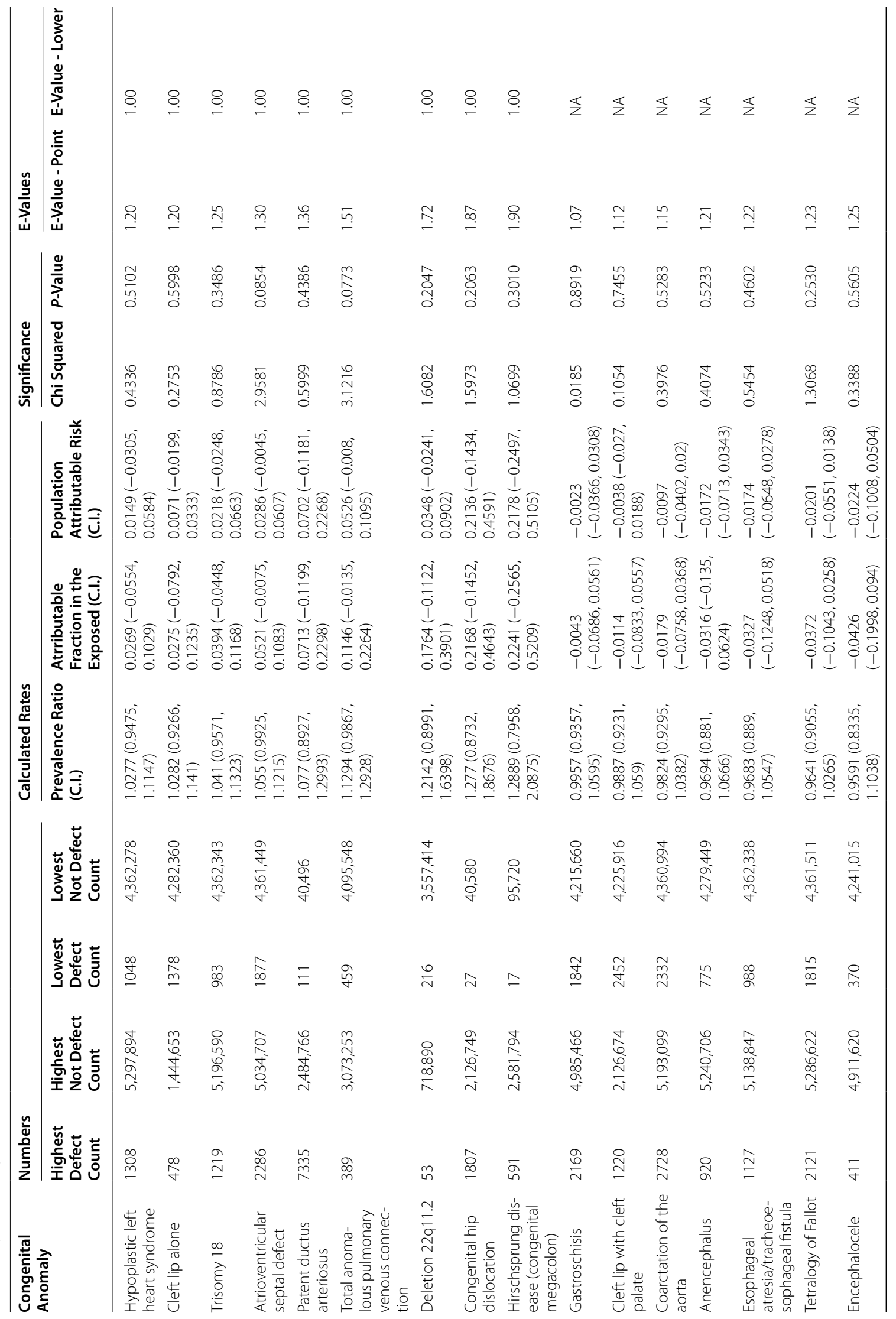




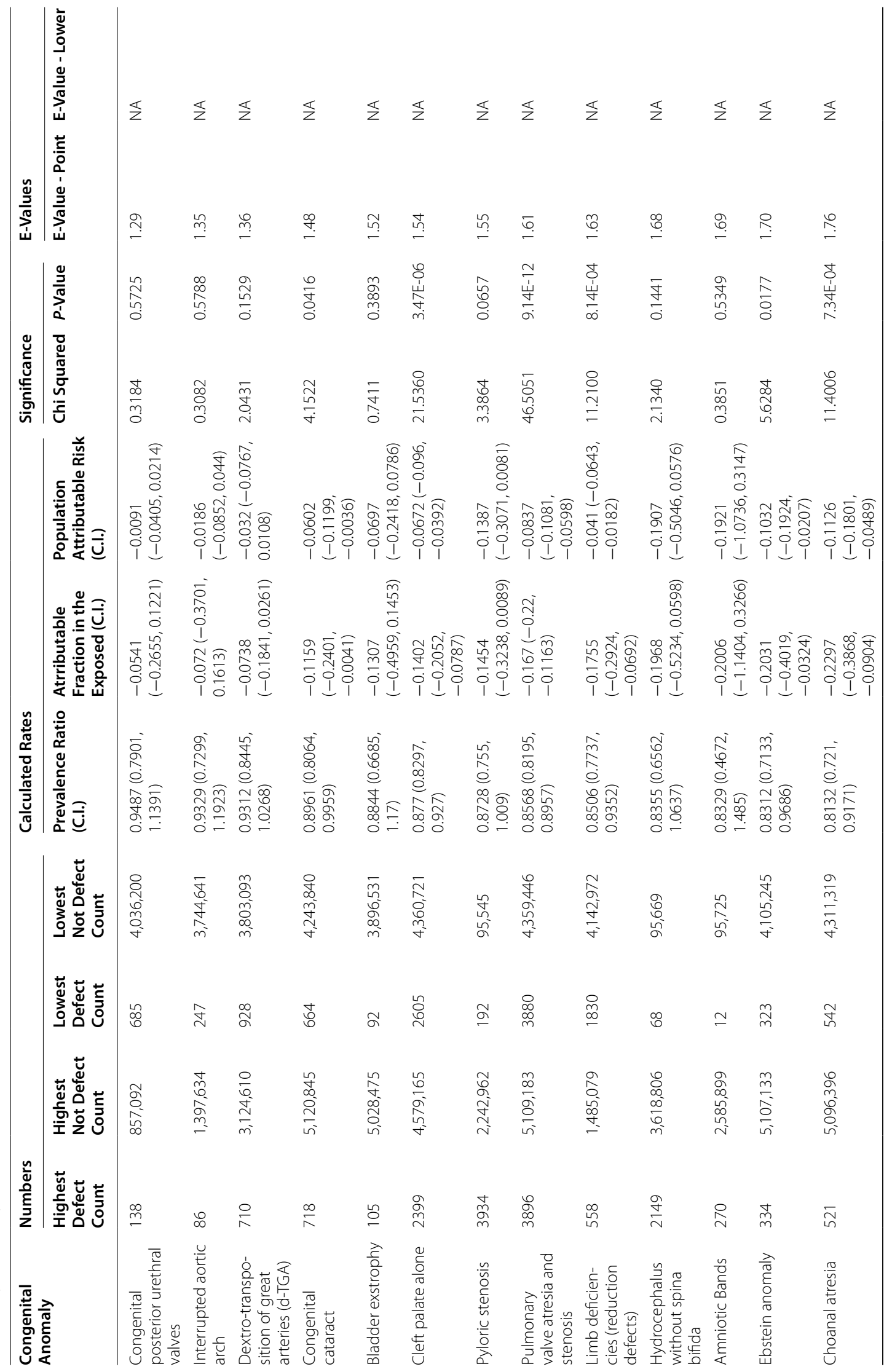




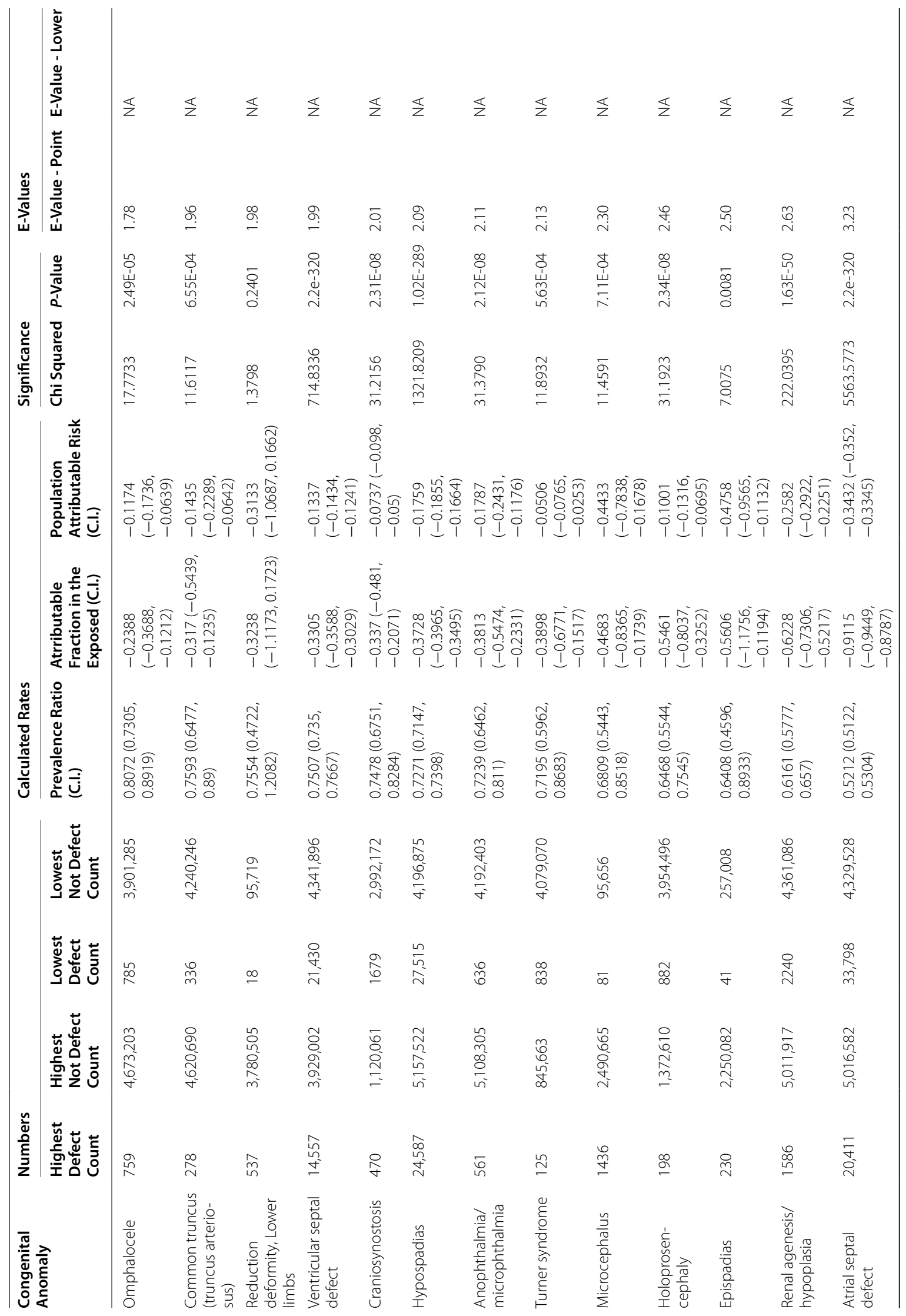




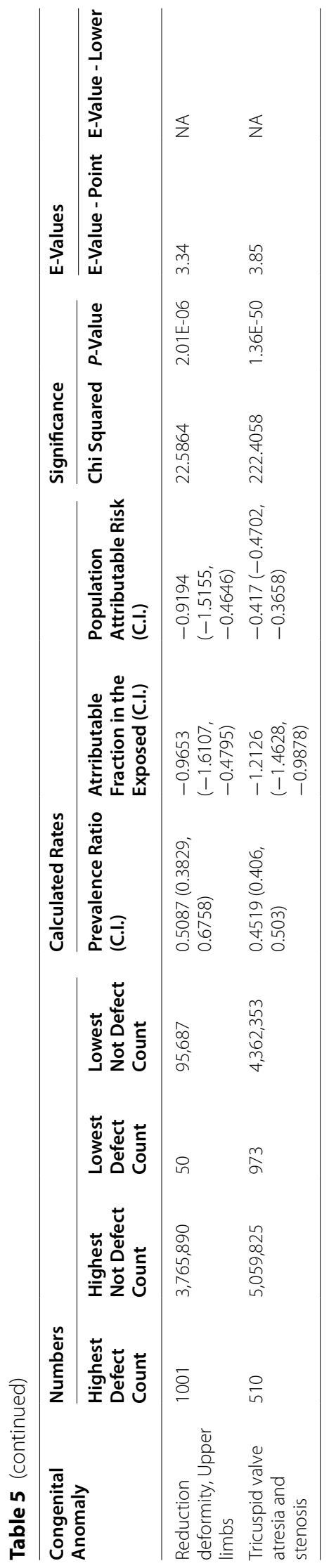


Table 6 Prevalence Ratios by Substance

\begin{tabular}{|c|c|c|c|c|c|c|}
\hline Congenital Anomaly & $\begin{array}{l}\text { Cigarettes } \\
\text { Prevalence } \\
\text { Ratio }\end{array}$ & $\begin{array}{l}\text { Binge Alcohol } \\
\text { Prevalence } \\
\text { Ratio }\end{array}$ & $\begin{array}{l}\text { Analgesics } \\
\text { Prevalence } \\
\text { Ratio }\end{array}$ & Ccoaine Prevalence Ratio & $\begin{array}{l}\text { Cannabis } \\
\text { Prevalence } \\
\text { Ratio }\end{array}$ & $\begin{array}{l}\text { Cannabidiol } \\
\text { Prevalence } \\
\text { Ratio }\end{array}$ \\
\hline Obstructive genitourinary defect & $0.92(0.87,0.97)$ & $1.02(0.97,1.07)$ & $0.9(0.85,0.94)$ & $1.17(1.11,1.23)$ & $0.86(0.82,0.9)$ & $1.92(1.63,2.27)$ \\
\hline $\begin{array}{l}\text { Cleft lip with and without cleft } \\
\text { palate }\end{array}$ & $1.06(0.98,1.13)$ & $0.95(0.88,1.02)$ & $1.3(1.2,1.41)$ & $1.02(0.94,1.11)$ & $0.94(0.87,1.01)$ & $1.52(1.08,2.14)$ \\
\hline Pulmonary valve atresia & $1.06(0.91,1.22)$ & $0.45(0.38,0.54)$ & $1.64(1.46,1.85)$ & $0.97(0.86,1.1)$ & $1.27(1.14,1.43)$ & $1.35(1.18,1.55)$ \\
\hline Cloacal exstrophy & $2.84(2.44,3.31)$ & $0.85(0.73,0.98)$ & $1.61(1.41,1.83)$ & $0.63(0.54,0.72)$ & $4.85(4.08,5.77)$ & $1.3(1.12,1.51)$ \\
\hline $\begin{array}{l}\text { Hirschsprung disease (congenital } \\
\text { megacolon) }\end{array}$ & $1.12(0.95,1.33)$ & $0.57(0.47,0.7)$ & $1.06(0.89,1.27)$ & $1.01(0.84,1.21)$ & $1.46(1.24,1.72)$ & $1.29(0.8,2.09)$ \\
\hline Congenital hip dislocation & $0.93(0.84,1.04)$ & $1.09(0.97,1.24)$ & $0.95(0.85,1.06)$ & $1.85(1.65,2.07)$ & $2.28(2.08,2.51)$ & $1.28(0.87,1.87)$ \\
\hline Small intestinal atresia/stenosis & $0.86(0.77,0.97)$ & $0.87(0.77,0.97)$ & $1.09(0.99,1.2)$ & $1.1(1,1.21)$ & $1.22(1.12,1.33)$ & $1.26(1.14,1.39)$ \\
\hline Single ventricle & $0.84(0.66,1.05)$ & $0.46(0.36,0.59)$ & $1.07(0.93,1.23)$ & $0.81(0.7,0.93)$ & $1.21(1.06,1.39)$ & $1.22(1,1.5)$ \\
\hline Deletion 22q11.2 & $0.59(0.45,0.77)$ & $1.26(0.98,1.6)$ & $2.93(2.39,3.58)$ & $1.81(1.46,2.25)$ & $1.36(1.09,1.68)$ & $1.21(0.9,1.64)$ \\
\hline Biliary atresia & $1.02(0.87,1.2)$ & $0.63(0.51,0.77)$ & $0.88(0.74,1.05)$ & $1.12(0.94,1.32)$ & $1.19(1.02,1.39)$ & $1.2(1.02,1.4)$ \\
\hline Double outlet right ventricle & $1.04(0.92,1.17)$ & $0.69(0.6,0.79)$ & $1.12(1.01,1.23)$ & $0.91(0.82,1.01)$ & $1.19(1.08,1.31)$ & $1.16(1.01,1.33)$ \\
\hline Trisomy 13 & $0.62(0.53,0.71)$ & $0.97(0.85,1.1)$ & $0.86(0.82,0.9)$ & $1.76(1.67,1.85)$ & $1.29(1.23,1.35)$ & $1.14(1,1.28)$ \\
\hline $\begin{array}{l}\text { Total anomalous pulmonary } \\
\text { venous connection }\end{array}$ & $0.62(0.52,0.74)$ & $0.62(0.5,0.75)$ & $1.44(1.24,1.66)$ & $1.31(1.13,1.52)$ & $1.05(0.93,1.19)$ & $1.13(0.99,1.29)$ \\
\hline Clubfoot & $1.02(0.97,1.08)$ & $0.88(0.83,0.93)$ & $1.03(0.97,1.1)$ & $0.99(0.94,1.05)$ & $1.07(1.01,1.14)$ & $1.1(1.03,1.18)$ \\
\hline Diaphragmatic hernia & $1.22(1.13,1.33)$ & $0.83(0.76,0.91)$ & $1.15(1.06,1.26)$ & $0.87(0.79,0.95)$ & $1.24(1.15,1.34)$ & $1.09(1,1.17)$ \\
\hline Patent ductus arteriosus & $0.96(0.92,1)$ & $0.72(0.69,0.75)$ & $1.13(1.08,1.18)$ & $0.79(0.75,0.82)$ & $0.58(0.56,0.6)$ & $1.08(0.89,1.3)$ \\
\hline Trisomy 21 (Down syndrome) & $0.83(0.8,0.87)$ & $1.03(0.99,1.07)$ & $1.02(0.99,1.04)$ & $1.13(1.11,1.16)$ & $1.14(1.12,1.17)$ & $1.06(1.02,1.09)$ \\
\hline Atrioventricular septal defect & $0.95(0.89,1.01)$ & $0.79(0.74,0.84)$ & $1.07(1.01,1.13)$ & $0.92(0.87,0.98)$ & $1.05(1,1.11)$ & $1.06(0.99,1.12)$ \\
\hline Trisomy 18 & $0.66(0.6,0.73)$ & $0.89(0.82,0.98)$ & $1.06(1.02,1.1)$ & $1.34(1.29,1.39)$ & $1.31(1.27,1.35)$ & $1.04(0.96,1.13)$ \\
\hline Cleft lip alone & $1.06(0.96,1.17)$ & $1.04(0.94,1.15)$ & $1.18(1.08,1.28)$ & $0.82(0.75,0.89)$ & $0.97(0.9,1.05)$ & $1.03(0.93,1.14)$ \\
\hline Hypoplastic left heart syndrome & $1.2(1.1,1.3)$ & $0.69(0.62,0.75)$ & $1.12(1.04,1.19)$ & $0.91(0.85,0.98)$ & $1.1(1.03,1.17)$ & $1.03(0.95,1.11)$ \\
\hline Aortic valve stenosis & $0.96(0.87,1.06)$ & $0.71(0.64,0.8)$ & $1.59(1.48,1.71)$ & $1.03(0.96,1.11)$ & $0.9(0.84,0.96)$ & $1.02(0.93,1.12)$ \\
\hline Transposition of great arteries & $1.25(1.16,1.36)$ & $0.8(0.73,0.87)$ & $1.23(1.14,1.32)$ & $0.85(0.79,0.91)$ & $1.01(0.94,1.09)$ & $1.01(0.94,1.09)$ \\
\hline Anotia/microtia & $0.37(0.32,0.42)$ & $0.87(0.77,0.97)$ & $1.38(1.3,1.46)$ & $1.62(1.53,1.71)$ & $1(0.96,1.05)$ & $1.01(0.92,1.1)$ \\
\hline $\begin{array}{l}\text { Rectal and large intestinal atresia/ } \\
\text { stenosis }\end{array}$ & $1.2(1.12,1.28)$ & $0.84(0.78,0.9)$ & $1.08(1,1.16)$ & $0.87(0.81,0.94)$ & $0.9(0.85,0.96)$ & $1(0.94,1.07)$ \\
\hline $\begin{array}{l}\text { Spina bifida without anencepha- } \\
\text { lus }\end{array}$ & $1.04(0.97,1.11)$ & $0.83(0.77,0.9)$ & $1.36(1.3,1.42)$ & $1(0.96,1.05)$ & $1.05(1.01,1.09)$ & $1(0.94,1.07)$ \\
\hline Gastroschisis & $1(0.94,1.07)$ & $0.89(0.83,0.96)$ & $1.47(1.39,1.56)$ & $0.97(0.91,1.03)$ & $0.93(0.88,0.98)$ & $1(0.94,1.06)$ \\
\hline Cleft lip with cleft palate & $1.14(1.06,1.22)$ & $0.86(0.79,0.93)$ & $1.37(1.28,1.46)$ & $0.93(0.87,0.99)$ & $0.96(0.9,1.02)$ & $0.99(0.92,1.06)$ \\
\hline Coarctation of the aorta & $1.16(1.09,1.23)$ & $0.7(0.66,0.75)$ & $1.16(1.1,1.22)$ & $0.87(0.82,0.92)$ & $1.38(1.32,1.45)$ & $0.98(0.93,1.04)$ \\
\hline Anencephalus & $0.89(0.81,0.99)$ & $0.7(0.63,0.78)$ & $1.36(1.29,1.42)$ & $1.03(0.99,1.08)$ & $0.93(0.89,0.96)$ & $0.97(0.88,1.07)$ \\
\hline $\begin{array}{l}\text { Esophageal atresia/tracheoe- } \\
\text { sophageal fistula }\end{array}$ & $1.08(0.99,1.18)$ & $1.06(0.97,1.16)$ & $1.04(0.95,1.14)$ & $1.01(0.92,1.1)$ & $1.11(1.02,1.21)$ & $0.97(0.89,1.05)$ \\
\hline Tetralogy of Fallot & $1.1(1.03,1.17)$ & $0.78(0.73,0.84)$ & $1.03(0.97,1.1)$ & $0.99(0.93,1.05)$ & $0.96(0.91,1.02)$ & $0.96(0.91,1.03)$ \\
\hline Encephalocele & $1.21(1.05,1.39)$ & $0.68(0.58,0.81)$ & $1.08(0.98,1.18)$ & $0.91(0.83,1)$ & $0.94(0.86,1.02)$ & $0.96(0.83,1.1)$ \\
\hline $\begin{array}{l}\text { Congenital posterior urethral } \\
\text { valves }\end{array}$ & $1.11(0.95,1.29)$ & $0.61(0.52,0.71)$ & $1.06(0.92,1.23)$ & $0.84(0.73,0.97)$ & $1.33(1.15,1.54)$ & $0.95(0.79,1.14)$ \\
\hline Interrupted aortic arch & $1.27(0.99,1.62)$ & $0.85(0.65,1.11)$ & $1.38(1.12,1.69)$ & $0.81(0.65,1)$ & $1.04(0.84,1.27)$ & $0.93(0.73,1.19)$ \\
\hline $\begin{array}{l}\text { Dextro-transposition of great } \\
\text { arteries (d-TGA) }\end{array}$ & $1.19(1.08,1.33)$ & $0.89(0.79,1)$ & $0.96(0.87,1.07)$ & $0.89(0.8,0.98)$ & $0.75(0.68,0.82)$ & $0.93(0.84,1.03)$ \\
\hline Congenital cataract & $0.93(0.83,1.04)$ & $0.91(0.81,1.02)$ & $1.09(0.97,1.22)$ & $0.97(0.87,1.08)$ & $0.94(0.86,1.04)$ & $0.9(0.81,1)$ \\
\hline Bladder exstrophy & $1.57(1.19,2.05)$ & $0.92(0.69,1.24)$ & $1.16(0.86,1.57)$ & $0.86(0.62,1.18)$ & $1.03(0.75,1.4)$ & $0.88(0.67,1.17)$ \\
\hline Cleft palate alone & $1.23(1.16,1.31)$ & $1.01(0.95,1.08)$ & $1.16(1.09,1.22)$ & $0.97(0.91,1.02)$ & $0.96(0.91,1.01)$ & $0.88(0.83,0.93)$ \\
\hline Pyloric stenosis & $1.72(1.63,1.82)$ & $0.35(0.32,0.38)$ & $1.9(1.8,2.01)$ & $0.66(0.62,0.71)$ & $0.89(0.84,0.95)$ & $0.87(0.75,1.01)$ \\
\hline $\begin{array}{l}\text { Pulmonary valve atresia and } \\
\text { stenosis }\end{array}$ & $1(0.95,1.05)$ & $0.71(0.67,0.75)$ & $1.02(0.97,1.07)$ & $0.87(0.83,0.92)$ & $0.88(0.84,0.92)$ & $0.86(0.82,0.9)$ \\
\hline
\end{tabular}


Table 6 (continued)

\begin{tabular}{|c|c|c|c|c|c|c|}
\hline Congenital Anomaly & $\begin{array}{l}\text { Cigarettes } \\
\text { Prevalence } \\
\text { Ratio }\end{array}$ & $\begin{array}{l}\text { Binge Alcohol } \\
\text { Prevalence } \\
\text { Ratio }\end{array}$ & $\begin{array}{l}\text { Analgesics } \\
\text { Prevalence } \\
\text { Ratio }\end{array}$ & Ccoaine Prevalence Ratio & $\begin{array}{l}\text { Cannabis } \\
\text { Prevalence } \\
\text { Ratio }\end{array}$ & $\begin{array}{l}\text { Cannabidiol } \\
\text { Prevalence } \\
\text { Ratio }\end{array}$ \\
\hline $\begin{array}{l}\text { Limb deficiencies (reduction } \\
\text { defects) }\end{array}$ & $1.09(1,1.19)$ & $0.87(0.79,0.95)$ & $1.07(1,1.15)$ & $0.85(0.79,0.91)$ & $0.77(0.73,0.82)$ & $0.85(0.77,0.94)$ \\
\hline $\begin{array}{l}\text { Hydrocephalus without spina } \\
\text { bifida }\end{array}$ & $1.34(1.22,1.47)$ & $1.03(0.94,1.14)$ & $0.87(0.8,0.95)$ & $0.97(0.89,1.06)$ & $1.05(0.97,1.12)$ & $0.84(0.66,1.06)$ \\
\hline Amniotic Bands & $0.95(0.67,1.33)$ & $0.65(0.49,0.86)$ & $1.49(1.04,2.13)$ & $0.67(0.44,1.03)$ & $0.84(0.58,1.23)$ & $0.83(0.47,1.49)$ \\
\hline Ebstein anomaly & $1.13(0.97,1.33)$ & $0.71(0.59,0.85)$ & $1.33(1.16,1.53)$ & $0.92(0.8,1.05)$ & $0.88(0.78,1.01)$ & $0.83(0.71,0.97)$ \\
\hline Choanal atresia & $1.4(1.23,1.58)$ & $1.02(0.9,1.17)$ & $0.83(0.74,0.93)$ & $0.88(0.78,0.99)$ & $0.78(0.69,0.87)$ & $0.81(0.72,0.92)$ \\
\hline Omphalocele & $1.19(1.07,1.31)$ & $0.79(0.71,0.87)$ & $1.07(1.01,1.15)$ & $0.85(0.79,0.91)$ & $0.92(0.87,0.98)$ & $0.81(0.73,0.89)$ \\
\hline $\begin{array}{l}\text { Common truncus (truncus } \\
\text { arteriosus) }\end{array}$ & $2.1(1.8,2.46)$ & $0.58(0.48,0.7)$ & $1.02(0.87,1.2)$ & $0.72(0.61,0.85)$ & $0.73(0.63,0.84)$ & $0.76(0.65,0.89)$ \\
\hline Reduction deformity, Lower limbs & $1.22(1.03,1.45)$ & $0.94(0.78,1.13)$ & $1.04(0.88,1.24)$ & $0.82(0.69,0.98)$ & $0.84(0.72,0.98)$ & $0.76(0.47,1.21)$ \\
\hline Ventricular septal defect & $1.19(1.17,1.22)$ & $0.84(0.82,0.86)$ & $0.85(0.83,0.87)$ & $0.79(0.77,0.81)$ & $0.74(0.73,0.76)$ & $0.75(0.73,0.77)$ \\
\hline Craniosynostosis & $1.27(1.14,1.42)$ & $0.61(0.54,0.69)$ & $1.12(1.01,1.24)$ & $0.98(0.89,1.08)$ & $0.54(0.5,0.59)$ & $0.75(0.68,0.83)$ \\
\hline Hypospadias & $1.59(1.56,1.62)$ & $0.87(0.85,0.89)$ & $0.98(0.96,1)$ & $1(0.98,1.02)$ & $0.74(0.72,0.75)$ & $0.73(0.71,0.74)$ \\
\hline Anophthalmia/microphthalmia & $0.79(0.69,0.91)$ & $1.03(0.9,1.18)$ & $1.18(1.08,1.27)$ & $1.17(1.07,1.27)$ & $0.59(0.55,0.63)$ & $0.72(0.65,0.81)$ \\
\hline Turner syndrome & $0.61(0.53,0.71)$ & $1.14(1.01,1.3)$ & $1.01(0.95,1.08)$ & $1.11(1.04,1.19)$ & $1.57(1.47,1.66)$ & $0.72(0.6,0.87)$ \\
\hline Microcephalus & $1.32(1.18,1.46)$ & $0.78(0.7,0.87)$ & $1.1(0.98,1.23)$ & $0.91(0.82,1.02)$ & $0.71(0.65,0.78)$ & $0.68(0.54,0.85)$ \\
\hline Holoprosencephaly & $1.92(1.71,2.16)$ & $0.56(0.49,0.65)$ & $0.85(0.8,0.9)$ & $0.39(0.36,0.41)$ & $1.24(1.17,1.31)$ & $0.65(0.55,0.75)$ \\
\hline Epispadias & $0.8(0.62,1.04)$ & $1.22(0.92,1.62)$ & $0.69(0.53,0.91)$ & $1.37(1.04,1.8)$ & $1.31(1.03,1.67)$ & $0.64(0.46,0.89)$ \\
\hline Renal agenesis/hypoplasia & $1.25(1.17,1.34)$ & $0.92(0.86,0.99)$ & $0.95(0.9,1)$ & $0.84(0.8,0.89)$ & $0.54(0.51,0.57)$ & $0.62(0.58,0.66)$ \\
\hline Atrial septal defect & $2.53(2.49,2.57)$ & $0.56(0.54,0.57)$ & $1.31(1.29,1.34)$ & $0.71(0.7,0.73)$ & $0.71(0.7,0.72)$ & $0.52(0.51,0.53)$ \\
\hline Reduction deformity, Upper limbs & $0.9(0.79,1.03)$ & $0.95(0.83,1.09)$ & $1.02(0.9,1.16)$ & $0.83(0.73,0.94)$ & $0.7(0.63,0.79)$ & $0.51(0.38,0.68)$ \\
\hline $\begin{array}{l}\text { Tricuspid valve atresia and } \\
\text { stenosis }\end{array}$ & $0.67(0.59,0.76)$ & $0.91(0.81,1.03)$ & $0.61(0.56,0.68)$ & $1.17(1.07,1.29)$ & $0.53(0.49,0.58)$ & $0.45(0.41,0.5)$ \\
\hline Aniridia & $1.24(0.65,2.38)$ & $0.34(0.15,0.78)$ & $1.84(1.29,2.63)$ & $1.72(1.14,2.6)$ & $1.45(0.94,2.24)$ & - \\
\hline
\end{tabular}

which might be performed to investigate these data in greater detail. This brief analytical discussion is intended to be exemplary rather than exhaustive as a thorough spatiotemporal treatment of all of this material would require a very large undertaking indeed beyond the bounds of the space which is presently available.

\section{Small intestinal stenosis and atresia (SISA)}

We look first at small intestinal stenosis and atresia (SISA). Figure 16 presents map-graphically the states which provided data for this analysis. SISA is not diagnosed prenatally and is not impacted by ETOPFA practices.

Supplementary Table 13 presents the results of final inverse probability weighted mixed effects models. Interestingly one notes that in these models cannabis and / or cannabinoids are significantly related to SISA incidence. Importantly cannabidiol is independently significantly related and has a positive coefficient in all models in which it appears.

Supplementary Table 14 presents final inverse probability weighted robust generalized linear regression models.
Cannabis is significant alone. When all the substances are included in an additive model, only cannabis remains as shown in the second model on this page. In an interactive model with drugs cannabis is again independently significant. In comprehensive additive and interactive models including income and all ethnicities, significant terms including cannabidiol appear in both final models.

Supplementary Table 15 presents the results of inverse probability weighted panel regression models lagging cannabinoids. In both additive and interactive models terms including cannabidiol are significant and have positive coefficients.

States contributing data to the SISA dataset are shown in Supplementary Fig. 9 along with their edited geospatial linkages.

Table 15 presents the results of final geospatial models. Terms including cannabis are positive and significant in all cases.

Table 16 shows the results of final geospatial models looking at substances using the cannabinoids as covariates. In all cases terms including the cannabinoids are significant. In models lagged at one, two and three years 
Table 7 Attributable Fraction in the Exposed by Substance

\begin{tabular}{|c|c|c|c|c|c|c|}
\hline Congenital Anomaly & Cigarettes AFE & Binge Alcohol AFE & Analgesics AFE & Cocaine AFE & Cannabis AFE & Cannabidiol AFE \\
\hline $\begin{array}{l}\text { Obstructive genitouri- } \\
\text { nary defect }\end{array}$ & $-0.09(-0.15,-0.03)$ & $0.02(-0.03,0.07)$ & $-0.11(-0.17,-0.06)$ & $0.15(0.1,0.19)$ & $-0.16(-0.22,-0.12)$ & $0.48(0.39,0.56)$ \\
\hline $\begin{array}{l}\text { Cleft lip with and } \\
\text { without cleft palate }\end{array}$ & $0.05(-0.02,0.12)$ & $-0.06(-0.14,0.02)$ & $0.23(0.17,0.29)$ & $0.02(-0.06,0.1)$ & $-0.07(-0.15,0.01)$ & $0.34(0.07,0.53)$ \\
\hline Pulmonary valve atresia & $0.05(-0.09,0.18)$ & $-1.21(-1.63,-0.86)$ & $0.39(0.32,0.46)$ & $-0.03(-0.16,0.09)$ & $0.22(0.12,0.3)$ & $0.26(0.15,0.35)$ \\
\hline Cloacal exstrophy & $0.65(0.59,0.7)$ & $-0.18(-0.36,-0.02)$ & $0.38(0.29,0.45)$ & $-0.6(-0.84,-0.39)$ & $0.79(0.75,0.83)$ & $0.23(0.11,0.34)$ \\
\hline $\begin{array}{l}\text { Hirschsprung disease } \\
\text { (congenital mega- } \\
\text { colon) }\end{array}$ & $0.11(-0.06,0.25)$ & $-0.75(-1.12,-0.44)$ & $0.06(-0.12,0.21)$ & $0.01(-0.2,0.17)$ & $0.31(0.19,0.42)$ & $0.22(-0.26,0.52)$ \\
\hline $\begin{array}{l}\text { Congenital hip disloca- } \\
\text { tion }\end{array}$ & $-0.07(-0.19,0.04)$ & $0.09(-0.03,0.19)$ & $-0.05(-0.18,0.06)$ & $0.46(0.4,0.52)$ & $0.56(0.52,0.6)$ & $0.22(-0.15,0.46)$ \\
\hline $\begin{array}{l}\text { Small intestinal atresia/ } \\
\text { stenosis }\end{array}$ & $-0.16(-0.29,-0.03)$ & $-0.15(-0.29,-0.03)$ & $0.08(-0.01,0.16)$ & $0.09(0,0.17)$ & $0.18(0.11,0.25)$ & $0.21(0.12,0.28)$ \\
\hline Single ventricle & $-0.2(-0.51,0.05)$ & $-1.17(-1.77,-0.7)$ & $0.06(-0.08,0.19)$ & $-0.24(-0.43,-0.07)$ & $0.18(0.05,0.28)$ & $0.18(0,0.33)$ \\
\hline Deletion 22q11.2 & $-0.69(-1.2,-0.3)$ & $0.2(-0.02,0.38)$ & $0.66(0.58,0.72)$ & $0.45(0.31,0.56)$ & $0.26(0.09,0.41)$ & $0.18(-0.11,0.39)$ \\
\hline Biliary atresia & $0.02(-0.15,0.17)$ & $-0.6(-0.96,-0.31)$ & $-0.13(-0.34,0.05)$ & $0.1(-0.06,0.24)$ & $0.16(0.02,0.28)$ & $0.17(0.02,0.29)$ \\
\hline $\begin{array}{l}\text { Double outlet right } \\
\text { ventricle }\end{array}$ & $0.03(-0.09,0.15)$ & $-0.46(-0.67,-0.27)$ & $0.1(0.01,0.19)$ & $-0.1(-0.21,0.01)$ & $0.16(0.07,0.24)$ & $0.13(0.01,0.25)$ \\
\hline Trisomy 13 & $-0.62(-0.87,-0.41)$ & $-0.04(-0.18,0.09)$ & $-0.16(-0.23,-0.11)$ & $0.43(0.4,0.46)$ & $0.22(0.18,0.26)$ & $0.12(0,0.22)$ \\
\hline $\begin{array}{l}\text { Total anomalous } \\
\text { pulmonary venous } \\
\text { connection }\end{array}$ & $-0.6(-0.91,-0.34)$ & $-0.62(-0.98,-0.33)$ & $0.3(0.19,0.4)$ & $0.24(0.11,0.34)$ & $0.05(-0.08,0.16)$ & $0.11(-0.01,0.23)$ \\
\hline Clubfoot & $0.02(-0.04,0.07)$ & $-0.14(-0.2,-0.08)$ & $0.03(-0.03,0.09)$ & $-0.01(-0.07,0.05)$ & $0.07(0.01,0.12)$ & $0.09(0.03,0.15)$ \\
\hline Diaphragmatic hernia & $0.18(0.12,0.25)$ & $-0.21(-0.32,-0.1)$ & $0.13(0.05,0.2)$ & $-0.15(-0.26,-0.06)$ & $0.2(0.13,0.26)$ & $0.08(0,0.15)$ \\
\hline $\begin{array}{l}\text { Patent ductus arte- } \\
\text { riosus }\end{array}$ & $-0.05(-0.09,0)$ & $-0.39(-0.45,-0.33)$ & $0.11(0.07,0.15)$ & $-0.27(-0.33,-0.21)$ & $-0.72(-0.79,-0.65)$ & $0.07(-0.12,0.23)$ \\
\hline $\begin{array}{l}\text { Trisomy } 21 \text { (Down } \\
\text { syndrome) }\end{array}$ & $-0.2(-0.25,-0.15)$ & $0.03(-0.01,0.07)$ & $0.02(-0.01,0.04)$ & $0.12(0.1,0.14)$ & $0.12(0.11,0.14)$ & $0.05(0.02,0.09)$ \\
\hline $\begin{array}{l}\text { Atrioventricular septal } \\
\text { defect }\end{array}$ & $-0.06(-0.13,0.01)$ & $-0.27(-0.36,-0.18)$ & $0.06(0.01,0.12)$ & $-0.08(-0.15,-0.02)$ & $0.05(0,0.1)$ & $0.05(-0.01,0.11)$ \\
\hline Trisomy 18 & $-0.52(-0.67,-0.38)$ & $-0.12(-0.22,-0.02)$ & $0.05(0.02,0.09)$ & $0.25(0.23,0.28)$ & $0.24(0.21,0.26)$ & $0.04(-0.04,0.12)$ \\
\hline Cleft lip alone & $0.06(-0.04,0.15)$ & $0.04(-0.07,0.13)$ & $0.15(0.07,0.22)$ & $-0.23(-0.33,-0.13)$ & $-0.03(-0.12,0.04)$ & $0.03(-0.08,0.12)$ \\
\hline $\begin{array}{l}\text { Hypoplastic left heart } \\
\text { syndrome }\end{array}$ & $0.17(0.09,0.23)$ & $-0.46(-0.61,-0.32)$ & $0.1(0.04,0.16)$ & $-0.1(-0.18,-0.03)$ & $0.09(0.03,0.15)$ & $0.03(-0.06,0.1)$ \\
\hline Aortic valve stenosis & $-0.04(-0.16,0.06)$ & $-0.4(-0.57,-0.25)$ & $0.37(0.32,0.42)$ & $0.03(-0.04,0.1)$ & $-0.12(-0.19,-0.04)$ & $0.02(-0.07,0.11)$ \\
\hline $\begin{array}{l}\text { Transposition of great } \\
\text { arteries }\end{array}$ & $0.2(0.14,0.26)$ & $-0.25(-0.36,-0.14)$ & $0.18(0.12,0.24)$ & $-0.18(-0.27,-0.09)$ & $0.01(-0.06,0.08)$ & $0.01(-0.06,0.08)$ \\
\hline Anotia/microtia & $-1.73(-2.1,-1.4)$ & $-0.15(-0.29,-0.03)$ & $0.28(0.23,0.32)$ & $0.38(0.35,0.42)$ & $0(-0.04,0.04)$ & $0.01(-0.09,0.09)$ \\
\hline $\begin{array}{l}\text { Rectal and large intesti- } \\
\text { nal atresia/stenosis }\end{array}$ & $0.17(0.11,0.22)$ & $-0.19(-0.28,-0.11)$ & $0.07(0,0.14)$ & $-0.15(-0.24,-0.07)$ & $-0.11(-0.18,-0.04)$ & $0(-0.06,0.07)$ \\
\hline $\begin{array}{l}\text { Spina bifida without } \\
\text { anencephalus }\end{array}$ & $0.04(-0.03,0.1)$ & $-0.2(-0.3,-0.11)$ & $0.27(0.23,0.3)$ & $0(-0.05,0.04)$ & $0.05(0.01,0.08)$ & $0(-0.07,0.07)$ \\
\hline Gastroschisis & $0(-0.07,0.06)$ & $-0.13(-0.21,-0.05)$ & $0.32(0.28,0.36)$ & $-0.03(-0.1,0.03)$ & $-0.08(-0.14,-0.02)$ & $0(-0.07,0.06)$ \\
\hline $\begin{array}{l}\text { Cleft lip with cleft } \\
\text { palate }\end{array}$ & $0.12(0.06,0.18)$ & $-0.16(-0.26,-0.07)$ & $0.27(0.22,0.32)$ & $-0.08(-0.15,-0.01)$ & $-0.05(-0.11,0.02)$ & $-0.01(-0.08,0.06)$ \\
\hline $\begin{array}{l}\text { Coarctation of the } \\
\text { aorta }\end{array}$ & $0.14(0.09,0.19)$ & $-0.42(-0.52,-0.33)$ & $0.14(0.09,0.18)$ & $-0.15(-0.21,-0.09)$ & $0.28(0.24,0.31)$ & $-0.02(-0.08,0.04)$ \\
\hline Anencephalus & $-0.12(-0.24,-0.01)$ & $-0.43(-0.59,-0.28)$ & $0.26(0.23,0.3)$ & $0.03(-0.01,0.08)$ & $-0.08(-0.12,-0.04)$ & $-0.03(-0.14,0.06)$ \\
\hline $\begin{array}{l}\text { Esophageal atresia/ } \\
\text { tracheoesophageal } \\
\text { fistula }\end{array}$ & $0.07(-0.01,0.15)$ & $0.06(-0.03,0.14)$ & $0.04(-0.05,0.12)$ & $0.01(-0.09,0.09)$ & $0.1(0.02,0.17)$ & $-0.03(-0.12,0.05)$ \\
\hline Tetralogy of Fallot & $0.09(0.03,0.15)$ & $-0.28(-0.38,-0.19)$ & $0.03(-0.03,0.09)$ & $-0.01(-0.08,0.05)$ & $-0.04(-0.1,0.02)$ & $-0.04(-0.1,0.03)$ \\
\hline Encephalocele & $0.17(0.05,0.28)$ & $-0.46(-0.72,-0.24)$ & $0.07(-0.02,0.15)$ & $-0.1(-0.2,0)$ & $-0.07(-0.16,0.02)$ & $-0.04(-0.2,0.09)$ \\
\hline $\begin{array}{l}\text { Congenital posterior } \\
\text { urethral valves }\end{array}$ & $0.1(-0.06,0.23)$ & $-0.65(-0.93,-0.42)$ & $0.06(-0.08,0.18)$ & $-0.19(-0.38,-0.03)$ & $0.25(0.13,0.35)$ & $-0.05(-0.27,0.12)$ \\
\hline Interrupted aortic arch & $0.21(-0.01,0.38)$ & $-0.17(-0.54,0.1)$ & $0.27(0.11,0.41)$ & $-0.24(-0.53,0)$ & $0.04(-0.18,0.21)$ & $-0.07(-0.37,0.16)$ \\
\hline $\begin{array}{l}\text { Dextro-transposition of } \\
\text { great arteries ( } d-T G A)\end{array}$ & $0.16(0.07,0.25)$ & $-0.13(-0.27,0)$ & $-0.04(-0.15,0.06)$ & $-0.13(-0.25,-0.02)$ & $-0.34(-0.46,-0.22)$ & $-0.07(-0.18,0.03)$ \\
\hline
\end{tabular}


Table 7 (continued)

\begin{tabular}{|c|c|c|c|c|c|c|}
\hline Congenital Anomaly & Cigarettes AFE & Binge Alcohol AFE & Analgesics AFE & Cocaine AFE & Cannabis AFE & Cannabidiol AFE \\
\hline Congenital cataract & $-0.07(-0.2,0.04)$ & $-0.1(-0.24,0.02)$ & $0.08(-0.03,0.18)$ & $-0.03(-0.15,0.08)$ & $-0.06(-0.17,0.04)$ & $-0.12(-0.24,0)$ \\
\hline Bladder exstrophy & $0.36(0.16,0.51)$ & $-0.08(-0.45,0.19)$ & $0.14(-0.16,0.36)$ & $-0.17(-0.61,0.15)$ & $0.03(-0.33,0.29)$ & $-0.13(-0.5,0.15)$ \\
\hline Cleft palate alone & $0.19(0.14,0.23)$ & $0.01(-0.05,0.07)$ & $0.13(0.08,0.18)$ & $-0.04(-0.1,0.02)$ & $-0.05(-0.1,0.01)$ & $-0.14(-0.21,-0.08)$ \\
\hline Pyloric stenosis & $0.42(0.39,0.45)$ & $-1.85(-2.08,-1.63)$ & $0.47(0.44,0.5)$ & $-0.5(-0.61,-0.4)$ & $-0.12(-0.19,-0.05)$ & $-0.15(-0.32,0.01)$ \\
\hline $\begin{array}{l}\text { Pulmonary valve atresia } \\
\text { and stenosis }\end{array}$ & $0(-0.05,0.05)$ & $-0.4(-0.48,-0.33)$ & $0.02(-0.03,0.06)$ & $-0.14(-0.2,-0.09)$ & $-0.14(-0.19,-0.09)$ & $-0.17(-0.22,-0.12)$ \\
\hline $\begin{array}{l}\text { Limb deficiencies } \\
\text { (reduction defects) }\end{array}$ & $0.09(0,0.16)$ & $-0.15(-0.26,-0.05)$ & $0.07(0,0.13)$ & $-0.18(-0.26,-0.1)$ & $-0.29(-0.37,-0.22)$ & $-0.18(-0.29,-0.07)$ \\
\hline $\begin{array}{l}\text { Hydrocephalus without } \\
\text { spina bifida }\end{array}$ & $0.25(0.18,0.32)$ & $0.03(-0.06,0.12)$ & $-0.15(-0.25,-0.06)$ & $-0.03(-0.12,0.05)$ & $0.04(-0.03,0.11)$ & $-0.2(-0.52,0.06)$ \\
\hline Amniotic Bands & $-0.06(-0.49,0.25)$ & $-0.53(-1.03,-0.16)$ & $0.33(0.04,0.53)$ & $-0.49(-1.28,0.03)$ & $-0.19(-0.73,0.19)$ & $-0.2(-1.14,0.33)$ \\
\hline Ebstein anomaly & $0.12(-0.03,0.25)$ & $-0.41(-0.69,-0.18)$ & $0.25(0.14,0.35)$ & $-0.09(-0.25,0.05)$ & $-0.13(-0.29,0.01)$ & $-0.2(-0.4,-0.03)$ \\
\hline Choanal atresia & $0.28(0.19,0.37)$ & $0.02(-0.11,0.15)$ & $-0.2(-0.35,-0.07)$ & $-0.14(-0.28,-0.01)$ & $-0.29(-0.45,-0.15)$ & $-0.23(-0.39,-0.09)$ \\
\hline Omphalocele & $0.16(0.07,0.24)$ & $-0.27(-0.41,-0.14)$ & $0.07(0.01,0.13)$ & $-0.18(-0.26,-0.1)$ & $-0.09(-0.15,-0.02)$ & $-0.24(-0.37,-0.12)$ \\
\hline $\begin{array}{l}\text { Common truncus } \\
\text { (truncus arteriosus) }\end{array}$ & $0.52(0.44,0.59)$ & $-0.73(-1.1,-0.43)$ & $0.02(-0.15,0.16)$ & $-0.39(-0.64,-0.18)$ & $-0.37(-0.59,-0.18)$ & $-0.32(-0.54,-0.12)$ \\
\hline $\begin{array}{l}\text { Reduction deformity, } \\
\text { Lower limbs }\end{array}$ & $0.18(0.03,0.31)$ & $-0.07(-0.28,0.12)$ & $0.04(-0.14,0.19)$ & $-0.22(-0.46,-0.02)$ & $-0.2(-0.4,-0.02)$ & $-0.32(-1.12,0.17)$ \\
\hline $\begin{array}{l}\text { Ventricular septal } \\
\text { defect }\end{array}$ & $0.16(0.14,0.18)$ & $-0.19(-0.22,-0.16)$ & $-0.18(-0.2,-0.15)$ & $-0.26(-0.29,-0.23)$ & $-0.34(-0.37,-0.31)$ & $-0.33(-0.36,-0.3)$ \\
\hline Craniosynostosis & $0.21(0.12,0.29)$ & $-0.64(-0.84,-0.46)$ & $0.11(0.01,0.19)$ & $-0.02(-0.13,0.08)$ & $-0.84(-1.01,-0.69)$ & $-0.34(-0.48,-0.21)$ \\
\hline Hypospadias & $0.37(0.36,0.38)$ & $-0.15(-0.17,-0.13)$ & $-0.02(-0.04,0)$ & $0(-0.02,0.02)$ & $-0.35(-0.38,-0.33)$ & $-0.37(-0.4,-0.35)$ \\
\hline $\begin{array}{l}\text { Anophthalmia/micro- } \\
\text { phthalmia }\end{array}$ & $-0.26(-0.44,-0.1)$ & $0.03(-0.11,0.15)$ & $0.15(0.08,0.22)$ & $0.14(0.07,0.21)$ & $-0.71(-0.83,-0.59)$ & $-0.38(-0.55,-0.23)$ \\
\hline Turner syndrome & $-0.63(-0.89,-0.41)$ & $0.13(0.01,0.23)$ & $0.01(-0.05,0.07)$ & $0.1(0.04,0.16)$ & $0.36(0.32,0.4)$ & $-0.39(-0.68,-0.15)$ \\
\hline Microcephalus & $0.24(0.16,0.32)$ & $-0.28(-0.42,-0.15)$ & $0.09(-0.02,0.18)$ & $-0.09(-0.22,0.02)$ & $-0.41(-0.54,-0.28)$ & $-0.47(-0.84,-0.17)$ \\
\hline Holoprosencephaly & $0.48(0.41,0.54)$ & $-0.78(-1.05,-0.54)$ & $-0.18(-0.25,-0.11)$ & $-1.58(-1.74,-1.43)$ & $0.19(0.14,0.24)$ & $-0.55(-0.8,-0.33)$ \\
\hline Epispadias & $-0.24(-0.6,0.03)$ & $0.18(-0.08,0.38)$ & $-0.44(-0.9,-0.1)$ & $0.27(0.04,0.44)$ & $0.24(0.03,0.4)$ & $-0.56(-1.18,-0.12)$ \\
\hline $\begin{array}{l}\text { Renal agenesis/hypo- } \\
\text { plasia }\end{array}$ & $0.2(0.15,0.25)$ & $-0.08(-0.16,-0.01)$ & $-0.05(-0.11,0)$ & $-0.18(-0.25,-0.12)$ & $-0.86(-0.97,-0.76)$ & $-0.62(-0.73,-0.52)$ \\
\hline Atrial septal defect & $0.6(0.6,0.61)$ & $-0.79(-0.83,-0.76)$ & $0.24(0.22,0.25)$ & $-0.4(-0.43,-0.37)$ & $-0.4(-0.43,-0.38)$ & $-0.91(-0.94,-0.88)$ \\
\hline $\begin{array}{l}\text { Reduction deformity, } \\
\text { Upper limbs }\end{array}$ & $-0.11(-0.27,0.03)$ & $-0.05(-0.21,0.08)$ & $0.02(-0.11,0.14)$ & $-0.2(-0.36,-0.06)$ & $-0.43(-0.6,-0.27)$ & $-0.97(-1.61,-0.48)$ \\
\hline $\begin{array}{l}\text { Tricuspid valve atresia } \\
\text { and stenosis }\end{array}$ & $-0.49(-0.7,-0.31)$ & $-0.1(-0.24,0.03)$ & $-0.63(-0.8,-0.48)$ & $0.15(0.07,0.22)$ & $-0.88(-1.06,-0.72)$ & $-1.21(-1.46,-0.99)$ \\
\hline Aniridia & $0.2(-0.53,0.58)$ & $-1.92(-5.62,-0.28)$ & $0.46(0.23,0.62)$ & $0.42(0.12,0.61)$ & $0.31(-0.07,0.55)$ & - \\
\hline
\end{tabular}

terms including cannabidiol are significant and the coefficients positive.

Table 17 presents a similar analysis this time including all income and ethnicity covariates. In each model terms for the cannabinoids are positive and significant. In each model terms including cannabidiol are also positive and significant.

Table 18 collects some of the regression terms from earlier tables and presents their applicable computed E-Values for the inverse probability weighted mixed effects and panel models.

Table 19 performs a similar role for regression terms derived from geospatial models.

Supplementary Table 16 lists all 57 of these minimum E-Values in descending order. All 57 are noted to be above the threshold of $1.25,34$ are noted to be greater than 100 and 13 are infinite.
It is of interest to consider predicted values from geospatiotemporal models. For this purpose the comprehensive interactive model shown in Table 17 lagged to two years was chosen.

The 101 predicted percentile values from matrix multiplication and scale adjustment are shown graphically in Fig. 17 with least squares regression lines, cubic polynomial and GAM curves are fitted. Percentiles refer to percentiles of cannabidiol exposure. Supplementary Table 17 presents the comparison of the ninetieth and tenth percentiles, the 95th and fifth percentiles and the first and 99th percentiles. An increasing ratio is noted in the right hand column consistent with an increasing effect at higher doses, and the obvious upwards inflection point on the fitted curve.

Supplementary Table 18 presents concisely the results of the various linear, polynomial and GAM regressions. 
Table 8 Population Attributable Risk by Substance

\begin{tabular}{|c|c|c|c|c|c|c|}
\hline $\begin{array}{l}\text { Congenital } \\
\text { Anomaly }\end{array}$ & Cigarettes PAR & Binge Alcohol PAR & Analgesics PAR & Cocaine PAR & Cannabis PAR & Cannabidiol PAR \\
\hline $\begin{array}{l}\text { Obstructive geni- } \\
\text { tourinary defect }\end{array}$ & $-0.03(-0.05,-0.01)$ & $0.01(-0.02,0.03)$ & $-0.03(-0.05,-0.02)$ & $0.07(0.05,0.09)$ & $-0.04(-0.06,-0.03)$ & $0.47(0.38,0.55)$ \\
\hline $\begin{array}{l}\text { Cleft lip with and } \\
\text { without cleft palate }\end{array}$ & $0.02(-0.01,0.04)$ & $-0.02(-0.05,0.01)$ & $0.09(0.06,0.12)$ & $0.01(-0.03,0.05)$ & $-0.02(-0.04,0)$ & $0.34(0.07,0.53)$ \\
\hline $\begin{array}{l}\text { Hirschsprung } \\
\text { disease (congenital } \\
\text { megacolon) }\end{array}$ & $0.05(-0.02,0.11)$ & $-0.24(-0.32,-0.16)$ & $0.02(-0.04,0.08)$ & $0(-0.09,0.09)$ & $0.12(0.06,0.17)$ & $0.22(-0.25,0.51)$ \\
\hline $\begin{array}{l}\text { Congenital hip } \\
\text { dislocation }\end{array}$ & $-0.03(-0.08,0.02)$ & $0.05(-0.02,0.11)$ & $-0.02(-0.05,0.02)$ & $0.28(0.23,0.33)$ & $0.25(0.22,0.28)$ & $0.21(-0.14,0.46)$ \\
\hline $\begin{array}{l}\text { Pulmonary valve } \\
\text { atresia }\end{array}$ & $0.01(-0.02,0.05)$ & $-0.27(-0.32,-0.22)$ & $0.18(0.14,0.23)$ & $-0.02(-0.09,0.05)$ & $0.11(0.06,0.16)$ & $0.12(0.06,0.17)$ \\
\hline Biliary atresia & $0.01(-0.04,0.05)$ & $-0.16(-0.22,-0.1)$ & $-0.04(-0.1,0.01)$ & $0.06(-0.04,0.14)$ & $0.07(0.01,0.13)$ & $0.1(0.01,0.18)$ \\
\hline $\begin{array}{l}\text { Patent ductus } \\
\text { arteriosus }\end{array}$ & $-0.02(-0.04,0)$ & $-0.16(-0.18,-0.13)$ & $0.04(0.02,0.05)$ & $-0.12(-0.14,-0.09)$ & $-0.16(-0.17,-0.15)$ & $0.07(-0.12,0.23)$ \\
\hline Trisomy 13 & $-0.11(-0.13,-0.08)$ & $-0.01(-0.06,0.03)$ & $-0.06(-0.08,-0.04)$ & $0.3(0.27,0.32)$ & $0.11(0.09,0.12)$ & $0.07(0,0.13)$ \\
\hline $\begin{array}{l}\text { Small intestinal } \\
\text { atresia/stenosis }\end{array}$ & $-0.02(-0.04,-0.01)$ & $-0.05(-0.08,-0.01)$ & $0.03(0,0.06)$ & $0.06(0,0.11)$ & $0.1(0.06,0.14)$ & $0.06(0.03,0.09)$ \\
\hline Cloacal exstrophy & $0.27(0.22,0.31)$ & $-0.05(-0.09,-0.01)$ & $0.1(0.07,0.14)$ & $-0.23(-0.29,-0.16)$ & $0.57(0.51,0.62)$ & $0.06(0.02,0.1)$ \\
\hline Single ventricle & $-0.03(-0.06,0.01)$ & $-0.25(-0.31,-0.18)$ & $0.02(-0.03,0.07)$ & $-0.13(-0.22,-0.04)$ & $0.09(0.02,0.16)$ & $0.06(0,0.11)$ \\
\hline $\begin{array}{l}\text { Total anomalous } \\
\text { pulmonary venous } \\
\text { connection }\end{array}$ & $-0.08(-0.1,-0.05)$ & $-0.17(-0.23,-0.11)$ & $0.13(0.08,0.19)$ & $0.15(0.07,0.23)$ & $0.02(-0.04,0.08)$ & $0.05(-0.01,0.11)$ \\
\hline $\begin{array}{l}\text { Diaphragmatic } \\
\text { hernia }\end{array}$ & $0.05(0.03,0.08)$ & $-0.06(-0.1,-0.03)$ & $0.05(0.02,0.08)$ & $-0.08(-0.13,-0.03)$ & $0.09(0.06,0.12)$ & $0.04(0,0.08)$ \\
\hline $\begin{array}{l}\text { Double outlet right } \\
\text { ventricle }\end{array}$ & $0.01(-0.02,0.04)$ & $-0.15(-0.2,-0.1)$ & $0.04(0,0.07)$ & $-0.05(-0.11,0)$ & $0.09(0.04,0.14)$ & $0.04(0,0.07)$ \\
\hline Deletion 22q11.2 & $-0.11(-0.16,-0.06)$ & $0.09(-0.01,0.19)$ & $0.4(0.33,0.47)$ & $0.31(0.2,0.4)$ & $0.09(0.02,0.16)$ & $0.03(-0.02,0.09)$ \\
\hline $\begin{array}{l}\text { Trisomy } 21 \text { (Down } \\
\text { syndrome) }\end{array}$ & $-0.04(-0.05,-0.03)$ & $0.01(0,0.03)$ & $0.01(0,0.01)$ & $0.07(0.06,0.08)$ & $0.06(0.05,0.07)$ & $0.03(0.01,0.05)$ \\
\hline $\begin{array}{l}\text { Atrioventricular } \\
\text { septal defect }\end{array}$ & $-0.01(-0.03,0)$ & $-0.08(-0.11,-0.06)$ & $0.02(0,0.04)$ & $-0.04(-0.07,-0.01)$ & $0.02(0,0.04)$ & $0.03(0,0.06)$ \\
\hline Trisomy 18 & $-0.09(-0.11,-0.07)$ & $-0.04(-0.07,-0.01)$ & $0.02(0.01,0.04)$ & $0.16(0.14,0.18)$ & $0.11(0.1,0.13)$ & $0.02(-0.02,0.07)$ \\
\hline Clubfoot & $0.01(-0.01,0.02)$ & $-0.05(-0.06,-0.03)$ & $0.01(-0.01,0.02)$ & $0(-0.03,0.02)$ & $0.02(0,0.04)$ & $0.02(0,0.03)$ \\
\hline $\begin{array}{l}\text { Hypoplastic left } \\
\text { heart syndrome }\end{array}$ & $0.05(0.02,0.07)$ & $-0.13(-0.16,-0.1)$ & $0.04(0.02,0.07)$ & $-0.05(-0.09,-0.01)$ & $0.04(0.01,0.07)$ & $0.01(-0.03,0.06)$ \\
\hline Aortic valve stenosis & $-0.01(-0.04,0.01)$ & $-0.12(-0.15,-0.08)$ & $0.18(0.15,0.2)$ & $0.02(-0.02,0.06)$ & $-0.04(-0.07,-0.02)$ & $0.01(-0.04,0.06)$ \\
\hline Cleft lip alone & $0.01(-0.01,0.03)$ & $0.01(-0.02,0.05)$ & $0.06(0.03,0.1)$ & $-0.12(-0.17,-0.07)$ & $-0.02(-0.05,0.02)$ & $0.01(-0.02,0.03)$ \\
\hline $\begin{array}{l}\text { Transposition of } \\
\text { great arteries }\end{array}$ & $0.08(0.05,0.11)$ & $-0.08(-0.11,-0.05)$ & $0.07(0.05,0.1)$ & $-0.08(-0.12,-0.05)$ & $0(-0.02,0.03)$ & $0.01(-0.03,0.04)$ \\
\hline Anotia/microtia & $-0.19(-0.2,-0.17)$ & $-0.05(-0.09,-0.01)$ & $0.12(0.1,0.14)$ & $0.25(0.22,0.28)$ & $0(-0.02,0.02)$ & $0(-0.05,0.05)$ \\
\hline $\begin{array}{l}\text { Rectal and large } \\
\text { intestinal atresia/ } \\
\text { stenosis }\end{array}$ & $0.05(0.03,0.07)$ & $-0.06(-0.09,-0.04)$ & $0.02(0,0.05)$ & $-0.07(-0.11,-0.04)$ & $-0.04(-0.07,-0.02)$ & $0(-0.03,0.03)$ \\
\hline $\begin{array}{l}\text { Spina bifida without } \\
\text { anencephalus }\end{array}$ & $0.01(-0.01,0.03)$ & $-0.06(-0.09,-0.04)$ & $0.12(0.1,0.14)$ & $0(-0.02,0.02)$ & $0.02(0,0.04)$ & $0(-0.04,0.04)$ \\
\hline Gastroschisis & $0(-0.02,0.02)$ & $-0.04(-0.06,-0.02)$ & $0.15(0.12,0.17)$ & $-0.02(-0.05,0.02)$ & $-0.03(-0.05,-0.01)$ & $0(-0.04,0.03)$ \\
\hline $\begin{array}{l}\text { Cleft lip with cleft } \\
\text { palate }\end{array}$ & $0.03(0.01,0.04)$ & $-0.06(-0.09,-0.03)$ & $0.12(0.1,0.15)$ & $-0.04(-0.08,0)$ & $-0.02(-0.05,0.01)$ & $0(-0.03,0.02)$ \\
\hline $\begin{array}{l}\text { Congenital poste- } \\
\text { rior urethral valves }\end{array}$ & $0.03(-0.02,0.07)$ & $-0.17(-0.21,-0.12)$ & $0.02(-0.03,0.06)$ & $-0.09(-0.17,-0.02)$ & $0.09(0.04,0.13)$ & $-0.01(-0.04,0.02)$ \\
\hline $\begin{array}{l}\text { Coarctation of the } \\
\text { aorta }\end{array}$ & $0.04(0.02,0.05)$ & $-0.12(-0.14,-0.1)$ & $0.05(0.03,0.07)$ & $-0.08(-0.11,-0.05)$ & $0.14(0.12,0.15)$ & $-0.01(-0.04,0.02)$ \\
\hline Anencephalus & $-0.03(-0.05,0)$ & $-0.12(-0.16,-0.09)$ & $0.12(0.1,0.14)$ & $0.02(-0.01,0.04)$ & $-0.03(-0.05,-0.02)$ & $-0.02(-0.07,0.03)$ \\
\hline $\begin{array}{l}\text { Esophageal atresia/ } \\
\text { tracheoesophageal } \\
\text { fistula }\end{array}$ & $0.02(0,0.04)$ & $0.02(-0.01,0.06)$ & $0.01(-0.02,0.05)$ & $0(-0.05,0.05)$ & $0.04(0.01,0.08)$ & $-0.02(-0.06,0.03)$ \\
\hline
\end{tabular}


Table 8 (continued)

\begin{tabular}{|c|c|c|c|c|c|c|}
\hline $\begin{array}{l}\text { Congenital } \\
\text { Anomaly }\end{array}$ & Cigarettes PAR & Binge Alcohol PAR & Analgesics PAR & Cocaine PAR & Cannabis PAR & Cannabidiol PAR \\
\hline $\begin{array}{l}\text { Interrupted aortic } \\
\text { arch }\end{array}$ & $0.04(-0.01,0$ & $-0.05(-0.13,0.03)$ & $0.12(0.04,0.19)$ & $-0.13(-0.28,-0.01)$ & $0.02(-0.09,0.11)$ & $-0.02(-0.09,0.04)$ \\
\hline Tetralogy of Fallot & $0.02(0.01,0.04)$ & $-0.09(-0.11,-0.06)$ & $0.01(-0.01,0.04)$ & $-0.01(-0.04,0.03)$ & $-0.02(-0.04,0.01)$ & $-0.02(-0.06,0.01)$ \\
\hline Encephalocele & $0.05(0.01,0.09)$ & $-0.13(-0.18,-0.08)$ & $0.03(-0.01,0.06)$ & $-0.05(-0.1,0)$ & $-0.03(-0.06,0.01)$ & $-0.02(-0.1,0.05)$ \\
\hline $\begin{array}{l}\text { Dextro-transposi- } \\
\text { tion of great arteries } \\
\text { (d-TGA) }\end{array}$ & $0.04(0.02,0.07)$ & $-0.04(-0.08,0)$ & $-0.01(-0.05,0.02)$ & $-0.07(-0.14,-0.01)$ & $-0.13(-0.17,-0.09)$ & $-0.03(-0.08,0.01)$ \\
\hline $\begin{array}{l}\text { Limb deficiencies } \\
\text { (reduction defects) }\end{array}$ & $0.02(0,0.04)$ & $-0.05(-0.08,-0.02)$ & $0.03(0,0.05)$ & $-0.1(-0.14,-0.06)$ & $-0.13(-0.15,-0.1)$ & $-0.04(-0.06,-0.02)$ \\
\hline Turner syndrome & $-0.12(-0.15,-0.09)$ & $0.05(0,0.09)$ & $0(-0.02,0.02)$ & $0.06(0.02,0.1)$ & $0.12(0.1,0.14)$ & $-0.05(-0.08,-0.03)$ \\
\hline Congenital cataract & $-0.02(-0.04,0.01)$ & $-0.03(-0.08,0.01)$ & $0.03(-0.01,0.07)$ & $-0.02(-0.08,0.04)$ & $-0.02(-0.06,0.02)$ & $-0.06(-0.12,0)$ \\
\hline Cleft palate alone & $0.05(0.03,0.06)$ & $0.01(-0.02,0.03)$ & $0.05(0.03,0.08)$ & $-0.02(-0.05,0.01)$ & $-0.02(-0.04,0)$ & $-0.07(-0.1,-0.04)$ \\
\hline Bladder exstrophy & $0.13(0.04,0.21)$ & $-0.03(-0.13,0.07)$ & $0.05(-0.05,0.14)$ & $-0.07(-0.23,0.06)$ & $0.01(-0.1,0.11)$ & $-0.07(-0.24,0.08)$ \\
\hline Craniosynostosis & $0.03(0.01,0.04)$ & $-0.15(-0.18,-0.12)$ & $0.04(0,0.07)$ & $-0.01(-0.08,0.05)$ & $-0.25(-0.28,-0.22)$ & $-0.07(-0.1,-0.05)$ \\
\hline $\begin{array}{l}\text { Pulmonary valve } \\
\text { atresia and stenosis }\end{array}$ & $0(-0.01,0.01)$ & $-0.11(-0.13,-0.1)$ & $0.01(-0.01,0.02)$ & $-0.06(-0.09,-0.04)$ & $-0.04(-0.06,-0.03)$ & $-0.08(-0.11,-0.06)$ \\
\hline Holoprosencephaly & $0.15(0.12,0.18)$ & $-0.18(-0.22,-0.14)$ & $-0.06(-0.08,-0.04)$ & $-0.61(-0.65,-0.57)$ & $0.1(0.07,0.13)$ & $-0.1(-0.13,-0.07)$ \\
\hline Ebstein anomaly & $0.03(-0.01,0.07)$ & $-0.12(-0.17,-0.06)$ & $0.11(0.05,0.16)$ & $-0.05(-0.12,0.03)$ & $-0.05(-0.1,0)$ & $-0.1(-0.19,-0.02)$ \\
\hline Choanal atresia & $0.09(0.05,0.13)$ & $0.01(-0.04,0.06)$ & $-0.07(-0.11,-0.03)$ & $-0.07(-0.14,-0.01)$ & $-0.1(-0.15,-0.06)$ & $-0.11(-0.18,-0.05)$ \\
\hline Omphalocele & $0.04(0.02,0.07)$ & $-0.08(-0.12,-0.05)$ & & $-0.09(-0.13,-0.06)$ & $-0.04(-0.06,-0.01)$ & $-0.12(-0.17,-0.06)$ \\
\hline $\begin{array}{l}\text { Ventricular septal } \\
\text { defect }\end{array}$ & $0.06(0.05,0.06)$ & $-0.06(-0.07,-0.06)$ & $-0.05(-0.06,-0.04)$ & $-0.11(-0.12,-0.1)$ & $-0.08(-0.09,-0.07)$ & $-0.13(-0.14,-0.12)$ \\
\hline Pyloric stenosis & $0.24(0.21,0.26)$ & $-0.39(-0.41,-0.36)$ & $0.2(0.19,0.22)$ & $-0.16(-0.19,-0.14)$ & $-0.03(-0.04,-0.01)$ & $-0.14(-0.31,0.01)$ \\
\hline $\begin{array}{l}\text { Common truncus } \\
\text { (truncus arteriosus) }\end{array}$ & $0.22(0.17,0.27)$ & $-0.18(-0.24,-0.13)$ & $0.01(-0.05,0.06)$ & $-0.18(-0.28,-0.1)$ & $-0.13(-0.18,-0.07)$ & $-0.14(-0.23,-0.06)$ \\
\hline Hypospadias & $0.12(0.11,0.12)$ & $-0.05(-0.05,-0.04)$ & $-0.01(-0.01,0)$ & $0(-0.01,0.01)$ & $-0.13(-0.13,-0.12)$ & $-0.18(-0.19,-0.17)$ \\
\hline $\begin{array}{l}\text { Anophthalmia/ } \\
\text { microphthalmia }\end{array}$ & $-0.05(-0.09,-0.02)$ & $0.01(-0.04,0.06)$ & $0.06(0.03,0.09)$ & $0.08(0.04,0.13)$ & $-0.21(-0.23,-0.18)$ & $-0.18(-0.24,-0.12)$ \\
\hline $\begin{array}{l}\text { Hydrocephalus } \\
\text { without spina bifida }\end{array}$ & $0.09(0.06,0.12)$ & $0.01(-0.02,0.05)$ & $-0.04(-0.06,-0.02)$ & $-0.01(-0.05,0.02)$ & $0.01(-0.01,0.04)$ & $-0.19(-0.5,0.06)$ \\
\hline Amniotic Bands & $-0.01(-0.05,0.04)$ & $-0.14(-0.22,-0.06)$ & $0.1(0,0.2)$ & $-0.11(-0.22,-0.01)$ & $-0.03(-0.1,0.03)$ & $-0.19(-1.07,0.31)$ \\
\hline $\begin{array}{l}\text { Renal agenesis/ } \\
\text { hypoplasia }\end{array}$ & $0.06(0.04,0.08)$ & $-0.03(-0.05,0)$ & $-0.02(-0.03,0)$ & $-0.09(-0.12,-0.06)$ & $-0.23(-0.25,-0.22)$ & $-0.26(-0.29,-0.23)$ \\
\hline $\begin{array}{l}\text { Reduction deform- } \\
\text { ity, Lower limbs }\end{array}$ & $0.06(0,0.11)$ & $-0.02(-0.09,0.04)$ & $0.01(-0.05,0.07)$ & $-0.1(-0.19,-0.01)$ & $-0.05(-0.1,-0.01)$ & $-0.31(-1.07,0.17)$ \\
\hline Atrial septal defect & $0.28(0.27,0.28)$ & $-0.2(-0.21,-0.2)$ & $0.09(0.09,0.1)$ & $-0.17(-0.18,-0.16)$ & $-0.13(-0.14,-0.13)$ & $-0.34(-0.35,-0.33)$ \\
\hline $\begin{array}{l}\text { Tricuspid valve atre- } \\
\text { sia and stenosis }\end{array}$ & $-0.09(-0.12,-0.07)$ & $-0.03(-0.08,0.01)$ & $-0.16(-0.18,-0.13)$ & $0.09(0.04,0.13)$ & $-0.23(-0.26,-0.21)$ & $-0.42(-0.47,-0.37)$ \\
\hline Microcephalus & $0.11(0.07,0.16)$ & $-0.11(-0.16,-0.06)$ & $0.03(-0.01,0.06)$ & $-0.04(-0.09,0.01)$ & $-0.09(-0.12,-0.07)$ & $-0.44(-0.78,-0.17)$ \\
\hline Epispadias & $-0.09(-0.2,0.01)$ & $0.09(-0.04,0.2)$ & $-0.1(-0.17,-0.03)$ & $0.13(0.01,0.24)$ & $0.07(0,0.14)$ & $-0.48(-0.96,-0.11)$ \\
\hline $\begin{array}{l}\text { Reduction deform- } \\
\text { ity, Upper limbs }\end{array}$ & $-0.03(-0.07,0.01)$ & $-0.02(-0.07,0.03)$ & $0.01(-0.04,0.05)$ & $-0.09(-0.15,-0.03)$ & $-0.1(-0.13,-0.07)$ & $-0.92(-1.52,-0.46)$ \\
\hline Aniridia & $0.09(-0.22,0.32)$ & $-0.35(-0.57,-0.16)$ & $0.21(0.07,0.33)$ & $0.25(0.04,0.42)$ & $0.11(-0.04,0.24)$ & - \\
\hline
\end{tabular}

At Anova testing the cubic curve is noted to have a superior fit to the least squares regression line (Anova: $\mathrm{F}=365.64, \mathrm{df}=2,97, P=7.86 \times 10^{-47}$ ) and the GAM is also noted to have a superior fit to the least squares line (Anova: $\mathrm{F}=265.91, \mathrm{df}=7.89,91.11, P=2.83 \times 10^{-60}$ ). The GAM model was superior to the cubic model (Anova: $\left.\mathrm{F}=23.096, \mathrm{df}=5.85,93.15, P=3.37 \times 10^{-16}\right)$.
Supplementary Table 19 presents the E-Values which are applicable to these linear regression results. The minimum E-Values are noted to range up to $1.73 \times 10^{36}$.

As mentioned the abscissa of this regression study was percentiles of cannabidiol exposure. When percentiles of the three cannabinoids THC, cannabigerol and cannabidiol were used instead similar results were obtained 
Table 9 Significance Levels by Substance

\begin{tabular}{|c|c|c|c|c|c|c|}
\hline Congenital Anomaly & Cigarettes $P$-Value & $\begin{array}{l}\text { Binge } \\
\text { Alcohol } \\
P \text {-Value }\end{array}$ & Analgesics $P$-Value & Cocaine $P$-Value & Cannabis $P$-Value & Cannabidiol $P$-Value \\
\hline Atrial septal defect & $2.2 e-320$ & 0.0215 & $9.26 \mathrm{E}-39$ & 0.00536446 & 1.09E-08 & $2.2 \mathrm{e}-320$ \\
\hline Ventricular septal defect & 7.36E-08 & 0.1490 & $1.24 \mathrm{E}-20$ & $1.93 \mathrm{E}-04$ & 0.0033 & $2.2 e-320$ \\
\hline Hypospadias & $2.2 \mathrm{e}-320$ & 0.4362 & 0.0317 & 0.7536 & $1.38 \mathrm{E}-05$ & $1.02 E-289$ \\
\hline $\begin{array}{l}\text { Tricuspid valve atresia and } \\
\text { stenosis }\end{array}$ & $7.29 \mathrm{E}-10$ & 0.1377 & 4.01E-159 & $6.31 \mathrm{E}-04$ & 3.93E-04 & $1.36 \mathrm{E}-50$ \\
\hline Renal agenesis/hypoplasia & $5.30 \mathrm{E}-11$ & 0.0215 & 0.0606 & $2.82 \mathrm{E}-09$ & 0.0028 & $1.63 \mathrm{E}-50$ \\
\hline $\begin{array}{l}\text { Obstructive genitourinary } \\
\text { defect }\end{array}$ & 0.0012 & 0.4876 & $4.12 \mathrm{E}-05$ & 2.37E-09 & $3.92 \mathrm{E}-12$ & $2.22 \mathrm{E}-15$ \\
\hline $\begin{array}{l}\text { Pulmonary valve atresia and } \\
\text { stenosis }\end{array}$ & 0.9716 & 0.0408 & 0.4950 & $3.64 \mathrm{E}-08$ & $1.27 \mathrm{E}-08$ & $9.14 \mathrm{E}-12$ \\
\hline $\begin{array}{l}\text { Anophthalmia/microph- } \\
\text { thalmia }\end{array}$ & $8.86 \mathrm{E}-04$ & 0.6781 & 7.92E-05 & $3.62 \mathrm{E}-04$ & $1.48 \mathrm{E}-12$ & $2.12 \mathrm{E}-08$ \\
\hline Craniosynostosis & $1.80 \mathrm{E}-05$ & 1.0000 & 0.0266 & 0.7020 & $7.18 \mathrm{E}-05$ & $2.31 \mathrm{E}-08$ \\
\hline Holoprosencephaly & $1.66 \mathrm{E}-28$ & $2.00 \mathrm{E}-15$ & 5.89E-08 & $2.02 \mathrm{E}-04$ & $2.90 \mathrm{E}-12$ & $2.34 \mathrm{E}-08$ \\
\hline $\begin{array}{l}\text { Reduction deformity, Upper } \\
\text { limbs }\end{array}$ & 0.1301 & 0.4407 & 0.7110 & 0.0042 & 1.15E-09 & 2.01E-06 \\
\hline Cleft palate alone & $2.29 \mathrm{E}-11$ & 0.6532 & $3.78 \mathrm{E}-07$ & 0.2282 & 0.0892 & $3.47 \mathrm{E}-06$ \\
\hline $\begin{array}{l}\text { Small intestinal atresia/ } \\
\text { stenosis }\end{array}$ & 0.0106 & 0.0125 & 0.0775 & 0.0531 & 4.47E-06 & 5.93E-06 \\
\hline Pulmonary valve atresia & 0.4522 & 0.0978 & $2.23 \mathrm{E}-17$ & 0.6602 & $2.62 \mathrm{E}-05$ & $1.02 \mathrm{E}-05$ \\
\hline Omphalocele & $8.62 \mathrm{E}-04$ & $6.56 \mathrm{E}-06$ & 0.0288 & 7.27E-07 & 0.0056 & $2.49 \mathrm{E}-05$ \\
\hline Cloacal exstrophy & $1.36 \mathrm{E}-45$ & 0.0284 & $1.91 \mathrm{E}-12$ & $6.90 \mathrm{E}-11$ & $2.13 \mathrm{E}-86$ & $5.45 \mathrm{E}-04$ \\
\hline Turner syndrome & $9.14 \mathrm{E}-11$ & 0.0367 & 0.7522 & 0.0014 & $7.69 \mathrm{E}-49$ & 5.63E-04 \\
\hline $\begin{array}{l}\text { Common truncus (truncus } \\
\text { arteriosus) }\end{array}$ & $7.03 E-22$ & $1.02 \mathrm{E}-08$ & 0.8321 & 7.90E-05 & $2.51 \mathrm{E}-05$ & $6.55 \mathrm{E}-04$ \\
\hline Microcephalus & $3.39 \mathrm{E}-07$ & $7.86 \mathrm{E}-06$ & 0.1067 & 0.1136 & $1.90 \mathrm{E}-13$ & 7.11E-04 \\
\hline Choanal atresia & $1.42 \mathrm{E}-07$ & 0.7186 & 0.0014 & 0.0290 & $2.26 \mathrm{E}-05$ & 7.34E-04 \\
\hline $\begin{array}{l}\text { Limb deficiencies (reduction } \\
\text { defects) }\end{array}$ & 0.0428 & 0.0034 & 0.0405 & $1.72 \mathrm{E}-06$ & $2.33 \mathrm{E}-06$ & $8.14 \mathrm{E}-04$ \\
\hline Trisomy 21 (Down syndrome) & $1.75 \mathrm{E}-07$ & 0.0895 & 0.1491 & $1.49 \mathrm{E}-55$ & $4.02 E-26$ & 0.0021 \\
\hline Clubfoot & 0.4663 & $3.66 \mathrm{E}-06$ & 0.2788 & 0.7378 & 0.0136 & 0.0048 \\
\hline Epispadias & 0.0915 & 0.1591 & 0.0084 & 0.0242 & 0.0287 & 0.0081 \\
\hline $\begin{array}{l}\text { Cleft lip with and without } \\
\text { cleft palate }\end{array}$ & 0.1248 & 0.1441 & $3.43 \mathrm{E}-11$ & 0.5715 & 0.0887 & 0.0159 \\
\hline Ebstein anomaly & 0.1232 & $1.57 \mathrm{E}-04$ & $3.81 \mathrm{E}-05$ & 0.2279 & 0.0643 & 0.0177 \\
\hline Biliary atresia & 0.7657 & 4.48E-06 & 0.1548 & 0.2026 & 0.0244 & 0.0233 \\
\hline Diaphragmatic hernia & $5.26 \mathrm{E}-07$ & $5.72 \mathrm{E}-05$ & 0.0011 & 0.0013 & $2.11 \mathrm{E}-08$ & 0.0373 \\
\hline Double outlet right ventricle & 0.5739 & 4.13E-08 & 0.0314 & 0.0743 & 7.31E-04 & 0.0379 \\
\hline Congenital cataract & 0.2114 & 0.1134 & 0.1381 & 0.5892 & 0.2537 & 0.0416 \\
\hline Trisomy 13 & $1.77 \mathrm{E}-11$ & 0.5945 & $4.20 \mathrm{E}-09$ & $3.06 \mathrm{E}-106$ & $3.50 \mathrm{E}-06$ & 0.0427 \\
\hline Single ventricle & 0.1289 & $2.42 \mathrm{E}-10$ & 0.3626 & 0.0037 & 0.0060 & 0.0482 \\
\hline Pyloric stenosis & $1.38 \mathrm{E}-84$ & 0.1051 & $1.78 \mathrm{E}-122$ & $9.40 \mathrm{E}-29$ & 4.82E-04 & 0.0657 \\
\hline $\begin{array}{l}\text { Total anomalous pulmonary } \\
\text { venous connection }\end{array}$ & $1.05 \mathrm{E}-07$ & $1.64 \mathrm{E}-06$ & $9.84 \mathrm{E}-07$ & $3.78 \mathrm{E}-04$ & 0.4381 & 0.0773 \\
\hline Atrioventricular septal defect & 0.0937 & $6.48 \mathrm{E}-12$ & 0.0269 & 0.0073 & 0.0470 & 0.0854 \\
\hline $\begin{array}{l}\text { Hydrocephalus without spina } \\
\text { bifida }\end{array}$ & 1.84E-09 & 0.4705 & 0.0011 & 0.5085 & 0.2200 & 0.1441 \\
\hline $\begin{array}{l}\text { Dextro-transposition of great } \\
\text { arteries (d-TGA) }\end{array}$ & $8.25 \mathrm{E}-04$ & 0.0513 & 0.4822 & 0.0198 & $1.40 \mathrm{E}-10$ & 0.1529 \\
\hline Deletion 22q11.2 & $6.98 \mathrm{E}-05$ & 0.0672 & $3.67 E-28$ & $4.72 \mathrm{E}-08$ & 0.0051 & 0.2047 \\
\hline Congenital hip dislocation & 0.1991 & 0.1493 & 0.3536 & $1.13 \mathrm{E}-27$ & 7.27E-70 & 0.2063 \\
\hline
\end{tabular}


Table 9 (continued)

\begin{tabular}{|c|c|c|c|c|c|c|}
\hline Congenital Anomaly & Cigarettes $P$-Value & $\begin{array}{l}\text { Binge } \\
\text { Alcohol } \\
\text { P-Value }\end{array}$ & Analgesics $P$-Value & Cocaine $P$-Value & Cannabis $P$-Value & Cannabidiol $P$-Value \\
\hline $\begin{array}{l}\text { Reduction deformity, Lower } \\
\text { limbs }\end{array}$ & 0.0231 & 0.5017 & 0.6312 & 0.0277 & 0.0253 & 0.2401 \\
\hline Tetralogy of Fallot & 0.0047 & $7.04 \mathrm{E}-11$ & 0.2681 & 0.6597 & 0.1692 & 0.2530 \\
\hline $\begin{array}{l}\text { Hirschsprung disease (con- } \\
\text { genital megacolon) }\end{array}$ & 0.1818 & $1.55 \mathrm{E}-08$ & 0.4987 & 0.9565 & 6.69E-06 & 0.3010 \\
\hline Trisomy 18 & $6.05 \mathrm{E}-04$ & 0.0140 & 0.0034 & $2.43 \mathrm{E}-08$ & $1.06 \mathrm{E}-61$ & 0.3486 \\
\hline Bladder exstrophy & 0.0011 & 0.5975 & 0.3200 & 0.3343 & 0.8681 & 0.3893 \\
\hline Patent ductus arteriosus & 0.0375 & 0.8028 & $2.36 \mathrm{E}-07$ & $1.22 \mathrm{E}-09$ & $5.95 E-39$ & 0.4386 \\
\hline $\begin{array}{l}\text { Esophageal atresia/tracheoe- } \\
\text { sophageal fistula }\end{array}$ & 0.0977 & 0.2148 & 0.3880 & 0.8914 & 0.0195 & 0.4602 \\
\hline $\begin{array}{l}\text { Hypoplastic left heart } \\
\text { syndrome }\end{array}$ & $1.91 \mathrm{E}-05$ & $1.35 \mathrm{E}-14$ & 0.0014 & 0.0080 & 0.0048 & 0.5102 \\
\hline Anencephalus & 0.0269 & $1.45 \mathrm{E}-10$ & $4.05 E-44$ & 0.1442 & 7.15E-05 & 0.5233 \\
\hline Coarctation of the aorta & $5.48 \mathrm{E}-07$ & 0.8820 & $3.82 \mathrm{E}-08$ & $1.56 \mathrm{E}-07$ & $9.74 \mathrm{E}-45$ & 0.5283 \\
\hline Amniotic Bands & 0.7468 & 0.0026 & 0.0271 & 0.0681 & 0.3785 & 0.5349 \\
\hline Encephalocele & 0.0079 & 4.74E-06 & 0.1125 & 0.0397 & 0.1289 & 0.5605 \\
\hline $\begin{array}{l}\text { Congenital posterior urethral } \\
\text { valves }\end{array}$ & 0.2039 & $8.57 \mathrm{E}-11$ & 0.4012 & 0.0184 & $1.35 \mathrm{E}-04$ & 0.5725 \\
\hline Interrupted aortic arch & 0.0582 & 0.2411 & 0.0022 & 0.0472 & 0.7274 & 0.5788 \\
\hline Cleft lip alone & 0.2215 & 0.4773 & $1.95 \mathrm{E}-04$ & $1.40 \mathrm{E}-06$ & 0.4216 & 0.5998 \\
\hline Aortic valve stenosis & 0.4160 & $6.46 \mathrm{E}-09$ & $9.78 \mathrm{E}-36$ & 0.4210 & 0.0011 & 0.6318 \\
\hline Transposition of great arteries & $1.49 \mathrm{E}-08$ & $5.27 \mathrm{E}-07$ & $4.54 \mathrm{E}-08$ & $1.54 \mathrm{E}-05$ & 0.7669 & 0.7385 \\
\hline Cleft lip with cleft palate & $1.92 \mathrm{E}-04$ & $2.58 \mathrm{E}-04$ & $3.32 \mathrm{E}-04$ & 0.0304 & 0.1508 & 0.7455 \\
\hline Anotia/microtia & $9.45 \mathrm{E}-10$ & 0.0148 & $4.05 E-37$ & $2.02 \mathrm{E}-64$ & 0.9670 & 0.8853 \\
\hline Gastroschisis & 0.9978 & 0.0014 & $9.74 \mathrm{E}-13$ & 0.3662 & 0.0098 & 0.8919 \\
\hline $\begin{array}{l}\text { Rectal and large intestinal } \\
\text { atresia/stenosis }\end{array}$ & 4.34E-08 & $2.94 \mathrm{E}-06$ & 0.0493 & $1.69 \mathrm{E}-04$ & 0.0011 & 0.8966 \\
\hline $\begin{array}{l}\text { Spina bifida without anen- } \\
\text { cephalus }\end{array}$ & 0.2806 & $5.86 \mathrm{E}-06$ & $2.14 \mathrm{E}-29$ & 0.9854 & 0.0181 & 0.9332 \\
\hline Aniridia & 0.5068 & 0.0073 & $6.17 \mathrm{E}-04$ & 0.0096 & 0.0952 & - \\
\hline
\end{tabular}

particularly with relation to strongly sigmoidal modelled trends (results not shown).

\section{Obstructive genitourinary defects}

Figure 18 illustrates states contributing data to the obstructive genitourinary disorder (OGUD) dataset. This disorder is diagnosed prenatally but is not subject to ETOPFA practices.

Supplementary Table 20 presents final inverse probability weighted mixed effects models. Interestingly cannabis is again shown to be the only remaining term in the final additive model for drugs. In the last two models on the comprehensive dataset, the effect of cannabinoids is strongly positive. In the final comprehensive interactive model two significant terms include cannabidiol and have positive $\beta$-coefficients.
Final inverse probability weighted robust generalized linear regression models are presented in Supplementary Table 21. In the final comprehensive interactive model shown in this Table two terms for cannabidiol are strongly positive at high levels of statistical significance.

Final comprehensive inverse probability weighted panel regression models for cannabinoids are shown in Supplementary Table 22. Many positive terms for cannabinoids are noted.

Supplementary Fig. 10 illustrates the geospatial linkages which were derived and edited for the OGUD dataset.

Table 20 presents the results of final geospatiotemporal models for OGUD incidence. One notes that cannabis alone is highly signifcant. In an additive model limited to substance covariates, cannabis was the only remaining significant term in the final model. At two 
Table 10 E-Values by Substance

\begin{tabular}{|c|c|c|c|c|c|c|}
\hline Congenital Anomaly & $\begin{array}{l}\text { Cigarettes } \\
\text { E-Value }\end{array}$ & $\begin{array}{l}\text { Binge } \\
\text { Alcohol } \\
\text { E-Value }\end{array}$ & $\begin{array}{l}\text { Analgesics } \\
\text { E-Value }\end{array}$ & Cocaine E-Value & $\begin{array}{l}\text { Cannabis } \\
\text { E-Value }\end{array}$ & $\begin{array}{l}\text { Cannabidiol } \\
\text { E-Value }\end{array}$ \\
\hline Obstructive genitourinary defect & & 1.00 & & 1.46 & & 2.64 \\
\hline Pulmonary valve atresia & 1.00 & & 2.28 & & 1.53 & 1.64 \\
\hline Small intestinal atresia/stenosis & & & 1.00 & 1.00 & 1.49 & 1.54 \\
\hline Cloacal exstrophy & 4.32 & & 2.16 & & 7.61 & 1.48 \\
\hline Cleft lip with and without cleft palate & 1.00 & & 1.70 & 1.00 & & 1.37 \\
\hline Clubfoot & 1.00 & & 1.00 & & 1.14 & 1.20 \\
\hline Biliary atresia & 1.00 & & & 1.00 & 1.17 & 1.18 \\
\hline Trisomy 21 (Down syndrome) & & 1.00 & 1.00 & 1.46 & 1.49 & 1.16 \\
\hline Double outlet right ventricle & 1.00 & & 1.11 & & 1.36 & 1.10 \\
\hline Diaphragmatic hernia & 1.52 & & 1.31 & & 1.57 & 1.07 \\
\hline Trisomy 13 & & & & 2.73 & 1.75 & 1.07 \\
\hline Single ventricle & & & 1.00 & & 1.30 & 1.04 \\
\hline Transposition of great arteries & 1.59 & & 1.54 & & 1.00 & 1.00 \\
\hline Rectal and large intestinal atresia/stenosis & 1.50 & & 1.01 & & & 1.00 \\
\hline Hypoplastic left heart syndrome & 1.44 & & 1.26 & & 1.20 & 1.00 \\
\hline Cleft lip alone & 1.00 & 1.00 & 1.38 & & & 1.00 \\
\hline Hirschsprung disease (congenital megacolon) & 1.00 & & 1.00 & 1.00 & 1.77 & 1.00 \\
\hline Spina bifida without anencephalus & 1.00 & & 1.93 & 1.00 & 1.10 & 1.00 \\
\hline Anotia/microtia & & & 1.93 & 2.43 & 1.00 & 1.00 \\
\hline Aortic valve stenosis & & & 2.32 & 1.00 & & 1.00 \\
\hline Atrioventricular septal defect & & & 1.10 & & 1.03 & 1.00 \\
\hline Congenital hip dislocation & & 1.00 & & 2.69 & 3.57 & 1.00 \\
\hline Deletion 22q11.2 & & 1.00 & 4.22 & 2.28 & 1.42 & 1.00 \\
\hline Patent ductus arteriosus & & & 1.37 & & & 1.00 \\
\hline Total anomalous pulmonary venous connection & & & 1.79 & 1.51 & 1.00 & 1.00 \\
\hline Trisomy 18 & & & 1.16 & 1.91 & 1.85 & 1.00 \\
\hline Atrial septal defect & 4.38 & & 1.89 & & & \\
\hline Common truncus (truncus arteriosus) & 2.99 & & 1.00 & & & \\
\hline Holoprosencephaly & 2.80 & & & & 1.60 & \\
\hline Pyloric stenosis & 2.64 & & 3.00 & & & \\
\hline Hypospadias & 2.48 & & & & & \\
\hline Choanal atresia & 1.77 & 1.00 & & & & \\
\hline Hydrocephalus without spina bifida & 1.73 & 1.00 & & & 1.00 & \\
\hline Bladder exstrophy & 1.67 & & 1.00 & & 1.00 & \\
\hline Microcephalus & 1.65 & & 1.00 & & & \\
\hline Renal agenesis/hypoplasia & 1.62 & & & & & \\
\hline Ventricular septal defect & 1.61 & & & & & \\
\hline Cleft palate alone & 1.58 & 1.00 & 1.41 & & & \\
\hline Craniosynostosis & 1.53 & & 1.13 & & & \\
\hline Coarctation of the aorta & 1.42 & & 1.43 & & 1.97 & \\
\hline Dextro-transposition of great arteries (d-TGA) & 1.36 & & & & & \\
\hline Omphalocele & 1.35 & & 1.09 & & & \\
\hline Cleft lip with cleft palate & 1.33 & & 1.88 & & & \\
\hline Encephalocele & 1.28 & & 1.00 & & & \\
\hline Tetralogy of Fallot & 1.20 & & 1.00 & & & \\
\hline Reduction deformity, Lower limbs & 1.20 & & 1.00 & & & \\
\hline Limb deficiencies (reduction defects) & 1.06 & & 1.06 & & & \\
\hline Aniridia & 1.00 & & 1.90 & 1.53 & 1.00 & \\
\hline
\end{tabular}


Table 10 (continued)

\begin{tabular}{|c|c|c|c|c|c|c|}
\hline Congenital Anomaly & $\begin{array}{l}\text { Cigarettes } \\
\text { E-Value }\end{array}$ & $\begin{array}{l}\text { Binge } \\
\text { Alcohol } \\
\text { E-Value }\end{array}$ & $\begin{array}{l}\text { Analgesics } \\
\text { E-Value }\end{array}$ & Cocaine E-Value & $\begin{array}{l}\text { Cannabis } \\
\text { E-Value }\end{array}$ & $\begin{array}{l}\text { Cannabidiol } \\
\text { E-Value }\end{array}$ \\
\hline Congenital posterior urethral valves & 1.00 & & 1.00 & & 1.56 & \\
\hline Ebstein anomaly & 1.00 & & 1.60 & & & \\
\hline Esophageal atresia/tracheoesophageal fistula & 1.00 & 1.00 & 1.00 & 1.00 & 1.15 & \\
\hline Interrupted aortic arch & 1.00 & & 1.49 & & 1.00 & \\
\hline Amniotic Bands & & & 1.26 & & & \\
\hline Anencephalus & & & 1.91 & 1.00 & & \\
\hline Anophthalmia/microphthalmia & & 1.00 & 1.39 & 1.35 & & \\
\hline Congenital cataract & & & 1.00 & & & \\
\hline Epispadias & & 1.00 & & 1.25 & 1.20 & \\
\hline Gastroschisis & & & 2.12 & & & \\
\hline Pulmonary valve atresia and stenosis & & & 1.00 & & & \\
\hline Reduction deformity, Upper limbs & & & 1.00 & & & \\
\hline Tricuspid valve atresia and stenosis & & & & 1.35 & & \\
\hline Turner syndrome & & 1.10 & 1.00 & 1.25 & 2.31 & \\
\hline
\end{tabular}

years of lag cannabis was again the most significant term. The overall effect of cannabis in this model was positive. The effects of THC, cannabigerol and cannabidiol considered separately were positive in each case.

Table 21 shows the results of spatial and temporal lagging of cannabinoids. Several terms positive for cannabinoids are evident.

Table 22 lists final comprehensive interactive and interactive temporally lagged models. All models include positive significant terms for cannabinoids.

Table 23 lists the E-Values derived from mixed effects and panel regression models and Table 24 shows those derived from spatiotemporal models.

These 47 E-Values are listed in descending order in Supplementary Table 23. All 47 are noted to be above $1.25,36$ are noted to be above 100 and nine are noted to be infinite.

It is of interest to consider the way in which rising levels of cannabidiol might impact these results. The model chosen was the first comprehensive interactive model shown in Table 21 lagged to two years. Percentiles refer to percentiles of cannabidiol exposure.

The results of matrix multiplication and scale revision are shown in Fig. 19 with least squares regression lines, cubic polynomial and GAM curves fitted. Percentiles are compared in Supplementary Table 24 and one again notes an increasing ratio reflecting the obvious inflection points in the fitted curves. Regression summaries for these three smoothers are shown in Supplementary Table 25. At Anova testing both the cubic polynomial (Anova: $\mathrm{F}=499.86, \mathrm{df}=2,97, P=5.82 \times 10^{-51}$ ) and the GAM curve (Anova: $F=172.08, \mathrm{df}=7.7934,91.207$,
$P=1.61 \times 10^{-71}$ ) are noted to be superior to the least squares regression line confirming the significance of the inflection points in the curves.

The E-Values from the two linear regression models are shown in Supplementary Table 26 and their minima are noted to range up to $8.36 \times 10^{41}$ in the case of the cubic polynomial curve.

When this exercise was repeated for this congenital anomaly including percentiles of THC and cannabigerol in addition to cannabidiol exposure, again the sigmoidal non-linear shape of the fitted curve was strongly confirmed (results not shown).

\section{Discussion}

Main results

The overall picture to emerge from this national state level survey of cannabinoid teratogenesis confirms and extends the Hawaiian study of 2007 [13] in preference to the "standard model" of cannabinoid and cannabidiol teratogenesis widely canvassed in the medical profession. These findings support the genotoxic warnings placed by national regulatory agencies on approved cannabinoid products including cannabidiol.

The main outcome from this USA teratological survey and overview is that cannabis, THC, cannabidiol and cannabigerol have highly significant associations with congenital anomaly rates whether considered as continuous variables by regression line slope or categorical variables by comparing extreme quintiles and are accompanied by highly significant prevalence ratios, attributable fractions in the exposed, population attributable risks, significance levels and E-values. For the continuous variable analysis 


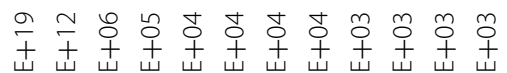

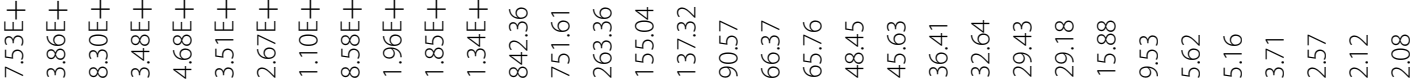

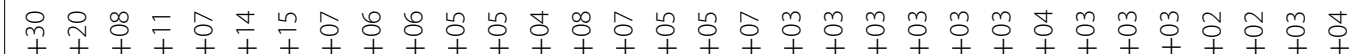

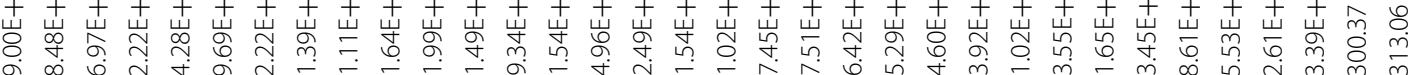

守

is

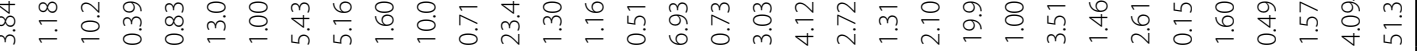

○ o

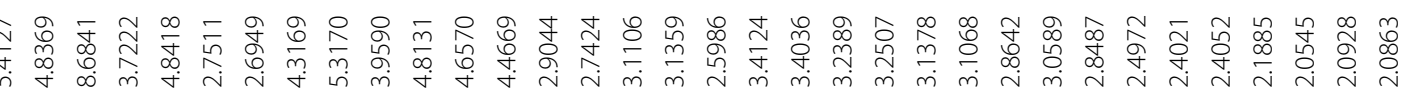

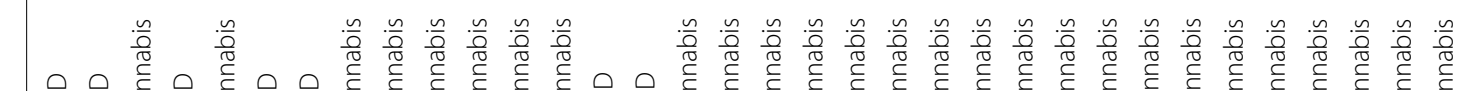

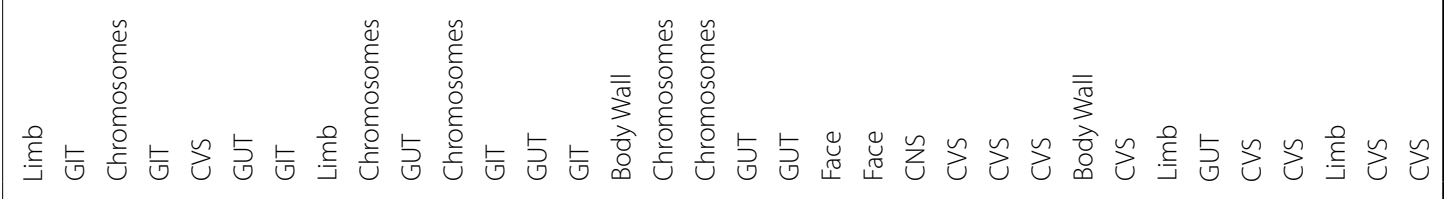




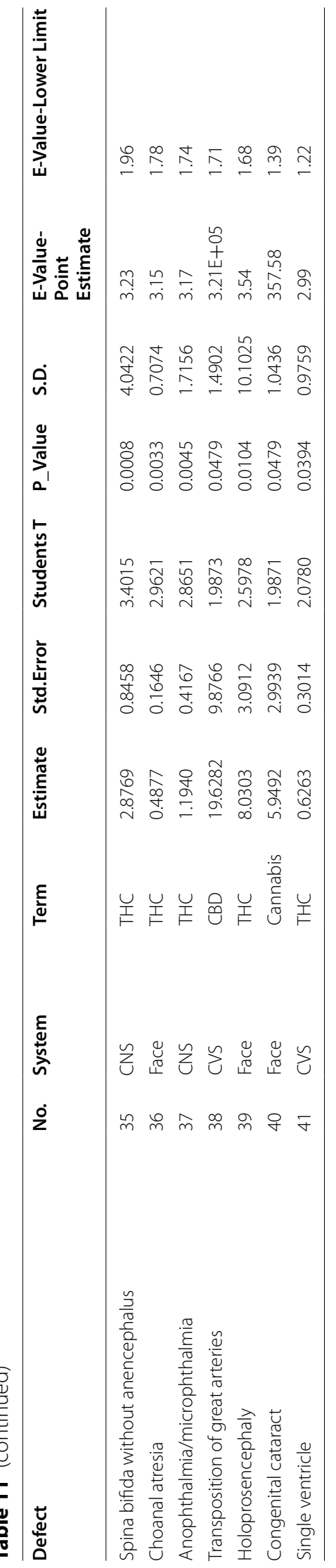


Table 12 Summary Continuous Variables by System

\begin{tabular}{llll}
\hline System & No. Anomalies & $\begin{array}{l}\text { Total No. } \\
\text { Anomalies }\end{array}$ & $\begin{array}{l}\text { \% of Total } \\
\text { Anomalies }\end{array}$ \\
\hline Chromosomes & 5 & 5 & $100.0 \%$ \\
GUT & 6 & 7 & $85.7 \%$ \\
GIT & 5 & 6 & $83.3 \%$ \\
Limb & 4 & 5 & $80.0 \%$ \\
Body Wall & 2 & 3 & $66.7 \%$ \\
CVS & 11 & 19 & $57.9 \%$ \\
Face & 5 & 9 & $55.6 \%$ \\
CNS & 3 & 7 & $42.9 \%$ \\
Total & $\mathbf{4 1}$ & $\mathbf{6 1}$ & $\mathbf{6 7 . 2 \%}$ \\
\hline
\end{tabular}

28 of the 41 CAs listed in Table 11 have minimum E-Values greater than 9.0 which is the very high value found in the tobacco-lung cancer relationship [86]. As judged by the number of ETOPFACARs impacted this putative teratogenic effect is greater for THC (40 CAs) than for cannabis (35 CAs) than for tobacco (11 CAs). For cannabidiol (11 CAs) this effect is greater than either last month alcohol consumption (5 CAs) or binge alcohol consumption (2 CAs). For two CAs considered in detail by spatiotemporal analysis and the formal techniques of causal inference, namely small intestinal stenosis or atresia and obstructive genitourinary defects, there is clear epidemiological evidence of both close association across time and space which persists after full model adjustment, and of a causal relationship with cannabinoid

Table 13 Summary CAs with Significant Cannabinoid E-Values Categorical Variables

\begin{tabular}{|c|c|c|c|c|c|c|c|c|c|}
\hline Defect & No. & System & Term & PR_C.I. & AFE_C.I. & ChiSqu & $P$-Value & $\begin{array}{l}\text { E-Value- } \\
\text { Point } \\
\text { Estimate }\end{array}$ & $\begin{array}{l}\text { E-Value- } \\
\text { Lower } \\
\text { Limit }\end{array}$ \\
\hline Cloacal exstrophy & 1 & GIT & Cannabis & $4.85(4.08,5.77)$ & $0.79(0.75,0.83)$ & 386.7336 & $2.13 \mathrm{E}-86$ & 9.17 & 7.61 \\
\hline Congenital hip dislocation & 2 & $\operatorname{Limb}$ & Cannabis & $2.28(2.08,2.51)$ & $0.56(0.52,0.60)$ & 310.8170 & $7.27 E-70$ & 3.99 & 3.57 \\
\hline Coarctation of the aorta & 3 & CVS & Cannabis & $1.38(1.31,1.45)$ & $0.28(0.24,0.31)$ & 152.3739 & $2.64 \mathrm{E}-35$ & 2.10 & 1.95 \\
\hline Obstructive genitourinary defect & 4 & GUT & $\mathrm{CBD}$ & $1.92(1.63,2.27)$ & $0.48(0.39,0.56)$ & 62.8480 & $2.22 \mathrm{E}-15$ & 3.25 & 2.64 \\
\hline Turner syndrome & 5 & Chromosomes & Cannabis & $1.54(1.36,1.75)$ & $0.35(0.26,0.43)$ & 46.5388 & $4.58 \mathrm{E}-12$ & 2.45 & 2.06 \\
\hline Trisomy 21 (Down syndrome) & 6 & Chromosomes & Cannabis & $1.12(1.08,1.16)$ & $0.11(0.08,0.14)$ & 45.1282 & $9.42 \mathrm{E}-12$ & 1.49 & 1.39 \\
\hline Diaphragmatic hernia & 7 & Body Wall & Cannabis & $1.24(1.15,1.34)$ & $0.20(0.13,0.26)$ & 31.3922 & $1.09 \mathrm{E}-08$ & 1.80 & 1.57 \\
\hline Trisomy 18 & 8 & Chromosomes & Cannabis & $1.22(1.13,1.32)$ & $0.18(0.11,0.24)$ & 25.4031 & $2.41 \mathrm{E}-07$ & 1.73 & 1.51 \\
\hline Small intestinal atresia/stenosis & 9 & GIT & Cannabis & $1.22(1.12,1.33)$ & $0.18(0.11,0.25)$ & 21.0508 & 2.33E-06 & 1.75 & 1.49 \\
\hline Small intestinal atresia/stenosis & 9 & GIT & CBD & $1.26(1.14,1.39)$ & $0.21(0.12,0.28)$ & 20.5107 & $5.93 \mathrm{E}-06$ & 1.83 & 1.54 \\
\hline $\begin{array}{l}\text { Hirschsprung disease (congenital } \\
\text { megacolon) }\end{array}$ & 10 & GIT & Cannabis & $1.46(1.24,1.72)$ & $0.31(0.19,0.42)$ & 20.2790 & $3.50 \mathrm{E}-06$ & 2.27 & 1.77 \\
\hline Pulmonary valve atresia & 11 & CVS & CBD & $1.35(1.18,1.55)$ & $0.26(0.15,0.35)$ & 19.4818 & $1.02 \mathrm{E}-05$ & 2.04 & 1.64 \\
\hline Holoprosencephaly & 12 & Face & Cannabis & $1.27(1.12,1.43)$ & $0.21(0.11,0.30)$ & 14.9227 & $5.94 \mathrm{E}-05$ & 1.86 & 1.50 \\
\hline Pulmonary valve atresia & 13 & CVS & Cannabis & $1.28(1.13,1.45)$ & $0.22(0.11,0.31)$ & 14.7343 & $6.56 \mathrm{E}-05$ & 1.87 & 1.50 \\
\hline Congenital posterior urethral valves & 14 & GUT & Cannabis & $1.33(1.15,1.54)$ & $0.25(0.13,0.35)$ & 14.5658 & $7.18 \mathrm{E}-05$ & 1.99 & 1.56 \\
\hline Cloacal exstrophy & 15 & GIT & CBD & $1.30(1.12,1.51)$ & $0.23(0.11,0.34)$ & 11.9548 & $5.45 \mathrm{E}-04$ & 1.92 & 1.48 \\
\hline Trisomy 13 & 16 & Chromosomes & Cannabis & $1.22(1.09,1.38)$ & $0.18(0.08,0.27)$ & 11.7980 & $3.18 \mathrm{E}-04$ & 1.75 & 1.41 \\
\hline Trisomy 21 (Down syndrome) & 17 & Chromosomes & CBD & $1.06(1.02,1.09)$ & $0.05(0.02,0.09)$ & 9.4889 & 0.0021 & 1.30 & 1.16 \\
\hline Double outlet right ventricle & 18 & CVS & Cannabis & $1.21(1.07,1.36)$ & $0.17(0.06,0.27)$ & 9.2314 & 0.0013 & 1.70 & 1.34 \\
\hline Clubfoot & 19 & Limb & $\mathrm{CBD}$ & $1.10(1.03,1.18)$ & $0.09(0.03,0.15)$ & 7.9686 & 0.0048 & 1.43 & 1.20 \\
\hline Deletion 22q11.2 & 20 & Chromosomes & Cannabis & $1.36(1.09,1.68)$ & $0.26(0.09,0.41)$ & 7.8339 & 0.0028 & 2.05 & 1.42 \\
\hline Clubfoot & 21 & Limb & Cannabis & $1.07(1.01,1.14)$ & $0.07(0.01,0.12)$ & 6.0907 & 0.0077 & 1.36 & 1.14 \\
\hline Cleft lip with and without cleft palate & 22 & Face & CBD & $1.52(1.08,2.14)$ & $0.34(0.07,0.53)$ & 5.8113 & 0.0159 & 2.41 & 1.37 \\
\hline $\begin{array}{l}\text { Esophageal atresia/tracheoesophageal } \\
\text { fistula }\end{array}$ & 23 & GIT & Cannabis & $1.11(1.02,1.21)$ & $0.10(0.02,0.17)$ & 5.4545 & 0.0112 & 1.45 & 1.15 \\
\hline Single ventricle & 24 & CVS & Cannabis & $1.23(1.03,1.46)$ & $0.19(0.03,0.32)$ & 5.4301 & 0.0113 & 1.76 & 1.22 \\
\hline Biliary atresia & 25 & GIT & CBD & $1.20(1.02,1.40)$ & $0.17(0.02,0.29)$ & 5.1462 & 0.0233 & 1.69 & 1.18 \\
\hline Biliary atresia & 25 & GIT & Cannabis & $1.19(1.02,1.39)$ & $0.16(0.02,0.28)$ & 5.0640 & 0.0141 & 1.67 & 1.17 \\
\hline Hypoplastic left heart syndrome & 26 & CVS & Cannabis & $1.10(1.01,1.19)$ & $0.09(0.01,0.16)$ & 4.8102 & 0.0164 & 1.42 & 1.11 \\
\hline Epispadias & 27 & GUT & Cannabis & $1.31(1.03,1.67)$ & $0.24(0.03,0.40)$ & 4.7877 & 0.0166 & 1.95 & 1.20 \\
\hline Diaphragmatic hernia & 28 & Body Wall & CBD & $1.09(1.00,1.17)$ & $0.08(0.00,0.15)$ & 4.3354 & 0.0373 & 1.39 & 1.07 \\
\hline Double outlet right ventricle & 29 & CVS & CBD & $1.16(1.01,1.33)$ & $0.14(0.01,0.25)$ & 4.3080 & 0.0379 & 1.58 & 1.10 \\
\hline Trisomy 13 & 30 & Chromosomes & CBD & $1.14(1.00,1.28)$ & $0.12(0.00,0.22)$ & 4.1053 & 0.0427 & 1.53 & 1.07 \\
\hline Single ventricle & 31 & CVS & CBD & $1.22(1.00,1.50)$ & $0.18(0.00,0.33)$ & 3.9021 & 0.0482 & 1.75 & 1.04 \\
\hline
\end{tabular}


Table 14 Summary Categorical Variables by System

\begin{tabular}{llll}
\hline System & No. Anomalies & $\begin{array}{l}\text { Total No. } \\
\text { Anomalies }\end{array}$ & $\begin{array}{l}\text { \% of Total } \\
\text { Anomalies }\end{array}$ \\
\hline Chromosomes & 5 & 5 & $100.0 \%$ \\
GIT & 5 & 6 & $83.3 \%$ \\
GUT & 3 & 7 & $42.9 \%$ \\
Limb & 2 & 5 & $40.0 \%$ \\
Body Wall & 1 & 3 & $33.3 \%$ \\
CVS & 5 & 19 & $26.3 \%$ \\
Face & 2 & 9 & $22.2 \%$ \\
CNS & 0 & 7 & $0.0 \%$ \\
Total & $\mathbf{2 2}$ & $\mathbf{6 1}$ & $\mathbf{3 6 . 1 \%}$ \\
\hline
\end{tabular}

including cannabidiol exposure. Moreover predictive modelling from selected spatiotemporal models demonstrates that the relationship between rising cannabidiol exposure and CA incidence is strongly sigmoidal in that both fitted curves show obvious strong positive inflections in their upper ranges which is closely and strongly reminiscent of the exponential dose-response curves observed in the laboratory in numerous genotoxic and mitochondriopathic assays [21, 24, 26, 31, 42, 54-65, 87]. $P$-values for this non-linearity are $2.83 \times 10^{-60}$ and $1.61 \times 10$ ${ }^{-71}$ respectively. For these CAs minimum polynomial E-Values for the predictive percentile models range up to $1.73 \times 10^{36}$ and $8.36 \times 10^{41}$.

The slope of the bivariate relationship between estimates of the ETOPFA-corrected CA incidence rate and the rate of substance exposure for many anomalies is significantly elevated for cannabis, THC and cannabidiol. As shown in Table 235 ETOPFA-corrected congenital anomalies have elevated minimum E-values by cannabis exposure regression slope which comprise nine cardiovascular anomalies, six anomalies of the urinary tract, five anomalies of the gastrointestinal tract, all five chromosomal anomalies, four limb musculoskeletal anomalies, two each of face and body wall anomalies and one brain anomaly. For 28 of these 35 anomalies the minimum E-Value is greater than 9.0. The forty CAs with elevated E-values after THC exposure may be grouped as ten cardiovascular CAs, six gastrointestinal CAs, six CAs of the urinary tract, all five chromosomal CAs, five CAs of the facial structures, four CAs of limb development including limb deficiencies and leg reductions, two central nervous system CAs including encephalocele and spina bifida without anencephalus, and two CAs of the body wall development diaphragmatic hernia and omphalocele (Supplementary Table 6).

The twelve ETOPFACARs with elevated E-Values from regression slopes after cannabidiol exposure include small and large intestinal esophageal and biliary atresias and stenoses, hip dislocation, obstructive genitourinary anomalies, and diaphragmatic herniae, cleft palate, reduction deformity of legs and transposition of the great arteries. Obstructive genitourinary defect, esophageal, small and large intestinal and biliary atresias and stenoses, diaphragmatic hernia, Hirschsprungs disease and hip dislocation have elevated E-Values when cannabidiol is considered as both continuous and categorical variables (Tables 3 and 5). For nine of these 12 CAs the minimum E-Value is greater than 18 (Table 3).

Tables 2 and 4 list the CAs with elevated E-Values when cannabis is treated as a continuous and as a categorical variable respectively. The defects which appear on both lists are the chromosomal anomalies Trisomies 13, 18 and 21 (Downs syndrome) and Deletion 22q11.2; the gastrointestinal anomalies esophageal atresia, small intestinal atresia or stenosis, biliary atresia and Hirschsprung disease; the cardiovascular defects hypoplastic left heart syndrome, coarctation of the aorta and pulmonary valve atresia or stenosis; the limb defects congenital hip dislocation and clubfoot, the body wall defect diaphragmatic hernia, and the urological disorder congenital posterior urethral valve.

\section{Interpretation}

Hence these data show not only close association between cannabinoid exposure and various CAs but clearly indicate the existence of a threshold effect above which the teratogenic impact dramatically increases, closely mirroring in patterns of human disease the amply documented threshold effects seen in cellular, molecular, genotoxic and epigenotoxic laboratory studies [21, 24, 26, $31,42,54-65,87]$.

The present study is intended to be introductory and pathfinding in the sense that its methods are not widely deployed across the published literature of the clinical teratological disciplines and we are keen to see advanced statistical methods more widely utilized to study the important questions raised by this study. However it is also true that sufficient evidence has been presented in the above material to enable several conclusions to be made definitively. Cannabinoid genotoxicity as tracked across multiple congenital anomalies is clinically significant and of public health importance and concern. Cannabis and cannabidiol test strongly positive on the bivariate results presented and are each implicated in more congenital anomalies than either tobacco or alcohol respectively both legal drugs which are widely acknowledged to be toxic to the developing foetus. Based on the very elevated minimum E-Values ofound cannabidiol is also a clinically significant teratogen and presumptive genotoxin and is more potent than either binge alcohol consumption or last month alcohol use. For selected 
Table 15 Small Intestinal Stenosis or Atresia - Introductory Space - Time Regression Models

\begin{tabular}{|c|c|c|c|c|c|c|}
\hline \multirow[t]{2}{*}{ Lagged Variables } & \multicolumn{3}{|l|}{ Parameter } & \multicolumn{3}{|c|}{ Model Parameters } \\
\hline & Parameter & Estimate (C.I.) & $P$-Value & Parameter & Value & Parameter $P$-Value \\
\hline & \multirow{2}{*}{\multicolumn{2}{|c|}{$\begin{array}{l}\text { Additive Model - Drugs } \\
\text { spreml(Rate } \sim \text { Cigarettes + Cannabis + anlyr + Binge.Alco- } \\
\text { hol + Cocaine) }\end{array}$}} & & S.D. & 0.4633 & \\
\hline & & & & LogLik & -112.1308 & \\
\hline & \multirow{2}{*}{ Cannabis } & \multirow[t]{2}{*}{$1.15(0.46,1.84)$} & \multirow[t]{2}{*}{0.0014} & psi & 0.8736 & $<2.2 \mathrm{e}-16$ \\
\hline & & & & lambda & -0.2041 & 0.04235 \\
\hline & \multirow{2}{*}{\multicolumn{3}{|c|}{$\begin{array}{l}\text { Interactive Model - Drugs } \\
\text { spreml(Rate } \sim \text { Cigarettes * Cannabis * anlyr * Binge.Alco- } \\
\text { hol+Cocaine) }\end{array}$}} & & & \\
\hline & & & & & & \\
\hline & Cigarettes: Cannabis: Binge.Alcohol & $57.95(30.14,85.75)$ & 4.41E-05 & S.D. & 0.8069 & \\
\hline & Cannabis: Binge.Alcohol & $30.95(15.37,46.53)$ & $9.90 \mathrm{E}-05$ & LogLik & -100.5249 & \\
\hline & $\begin{array}{l}\text { Cigarettes: Cannabis: Binge.Alco- } \\
\text { hol: Analgesics }\end{array}$ & $11.55(3.04,20.06)$ & 0.0078 & psi & 0.9063 & $<2.2 \mathrm{e}-16$ \\
\hline & Cigarettes: Analgesics & $-3.12(-5.07,-1.17)$ & 0.0018 & lambda & -0.2276 & 0.01861 \\
\hline & Cigarettes: Cannabis: Analgesics & $-3.96(-6.04,-1.88)$ & 0.0002 & & & \\
\hline & Cigarettes: Cannabis & $-13.09(-19.59,-6.59)$ & 7.87E-05 & & & \\
\hline & 2 Years Lag & & & & & \\
\hline & Interactive Model - Drugs & & & & & \\
\hline & $\begin{array}{l}\text { spreml(Rate Cigarettes * Cannabi } \\
\text { hol + Cocaine) }\end{array}$ & is * anlyr * Binge.Alco- & & & & \\
\hline \multirow[t]{8}{*}{ Cannabis, 2} & Cannabis: Analgesics & $68.51(39.94,97.07)$ & 2.60E-06 & S.D. & 0.4309 & \\
\hline & Cocaine & $-1.36(-2.18,-0.53)$ & 0.00126 & LogLik & -75.0846 & \\
\hline & Cigarettes: Cannabis: Analgesics & $-160.88(-236.65,-85.11)$ & $3.16 \mathrm{E}-05$ & psi & 0.8940 & $<2.2 \mathrm{e}-16$ \\
\hline & Cigarettes: Binge.Alcohol & $-159.19(-224.69,-93.7)$ & 1.90E-06 & rho & -0.5234 & $2.31 \mathrm{E}-05$ \\
\hline & $\begin{array}{l}\text { Cannabis: Analgesics: Binge. } \\
\text { Alcohol }\end{array}$ & $-170.52(-233.74,-107.31)$ & $1.24 \mathrm{E}-07$ & & & \\
\hline & 4 Years Lag & & & & & \\
\hline & Interactive Model - Drugs & & & & & \\
\hline & $\begin{array}{l}\text { spreml(Rate Cigarettes * Cannabi } \\
\text { hol + Cocaine) }\end{array}$ & is * anlyr * Binge.Alco- & & & & \\
\hline \multirow[t]{7}{*}{ Cannabis, 4} & Cigarettes: Analgesics & $418.42(221.76,615.07)$ & 3.04E-05 & S.D. & 0.4485 & \\
\hline & Cannabis: Analgesics & $1284.76(677.88,1891.64)$ & 3.34E-05 & LogLik & -19.5113 & \\
\hline & Cigarettes & $1335.95(704.65,1967.25)$ & 3.36E-05 & lambda & -0.7130 & $1.59 \mathrm{E}-06$ \\
\hline & Cannabis & $4106.59(2160.15,6053.02)$ & $3.55 \mathrm{E}-05$ & & & \\
\hline & Cigarettes: Cannabis & $\begin{array}{l}-17,101.54(-26,215.01 \\
-7988.07)\end{array}$ & 0.0002 & & & \\
\hline & Cigarettes: Cannabis: Analgesics & $-5380.17(-8221.58,-2538.76)$ & 0.0002 & & & \\
\hline & Analgesics & $-101.13(-144.83,-57.43)$ & $5.73 \mathrm{E}-06$ & & & \\
\hline
\end{tabular}

congenital anomalies cannabinoid teratogenicity persists after multivariable adjustment in inverse probability weighted models of causal inference, and after consideration in their inherently space-time context. For both congenital anomalies studied in detail spatiotemporal modelling shows strong evidence of a threshold effect above which the impacts of cannabidiol and cannabinoid teratogenicity are supra-linear, sigmoidal and greatly amplified.

These findings lead to the sobering conclusion that cannabinoid genotoxicity is of great public health importance to maternal-foetal and reproductive medicine in contrast to the fact that it appears to be largely missing from public health discourse to date where it is essentially overlooked.

Moreover given that the prevalence of cannabis use and cannabinoid exposure in the global community is clearly rising increasing cannabinoid exposure will not be related in simple linear fashion to increased congenital anomalies across a wide spectrum of developmental disorders, but the non-linearity of the relationship and the existence of clear thresholds for genotoxicity both 


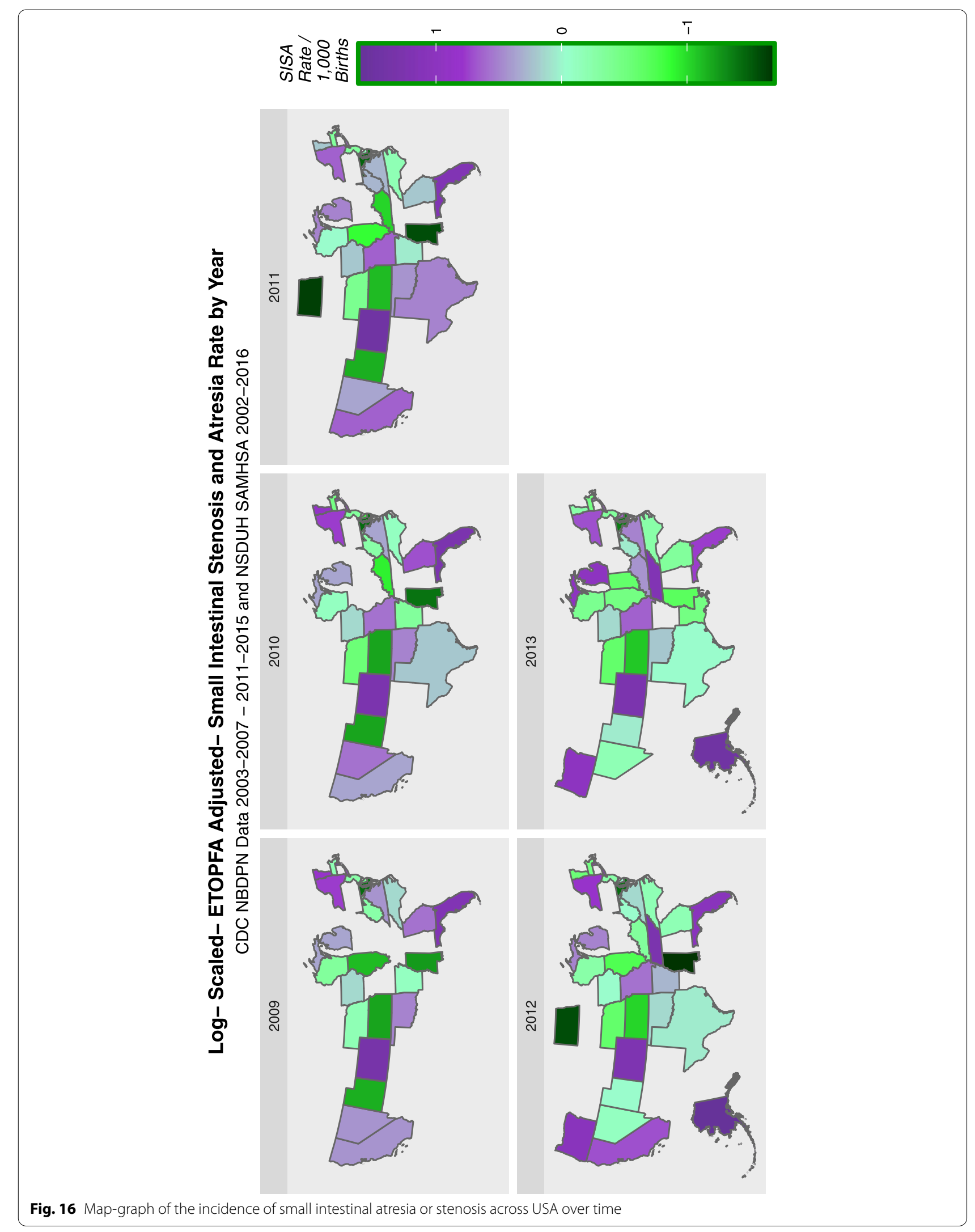


Table 16 Small Intestinal Stenosis or Atresia - Cannabinoid Space - Time Regression Models

\begin{tabular}{|c|c|c|c|c|c|c|}
\hline \multirow[t]{2}{*}{ Lagged Variables } & \multicolumn{3}{|l|}{ Parameter } & \multicolumn{3}{|c|}{ Model Parameters } \\
\hline & Parameter & Estimate (C.I.) & $P$-Value & Parameter & Value & Parameter $P$-Value \\
\hline & \multicolumn{6}{|l|}{ Additive Model - Cannabinoids } \\
\hline & \multicolumn{6}{|c|}{ spreml(Rate $\sim$ Cigarettes $+T H C+C B G+C B D+$ anlyr + Binge.Alcohol + Cocaine $)$} \\
\hline & CBG & $0.96221(0.28,1.64)$ & 0.0055 & S.D. & 0.4323 & \\
\hline & Binge.Alcohol & $8.50833(1.49,15.53)$ & 0.0175 & LogLik & -107.7976 & \\
\hline & $\mathrm{THC}$ & $-1.57158(-3.08,-0.06)$ & 0.0416 & psi & 0.9129 & $<2.2 \mathrm{e}-16$ \\
\hline & Cigarettes & $-6.73252(-13.04,-0.43)$ & 0.0363 & rho & -0.2431 & 0.01896 \\
\hline & \multicolumn{6}{|l|}{ Interactive Model-Cannabinoids } \\
\hline & \multicolumn{6}{|c|}{ spreml(Rate Cigarettes * THC *CBG *CBD+anlyr + Binge.Alcohol+ Cocaine) } \\
\hline & Cigarettes: THC: Binge.Alcohol & $5169.433(3191.79,7147.08)$ & 3.00E-07 & S.D. & 0.6566 & \\
\hline & THC & $172.247(93.57,250.92)$ & $1.78 \mathrm{E}-05$ & LogLik & -87.0831 & \\
\hline & Cigarettes & $1748.111(926.38,2569.84)$ & 3.05E-05 & psi & 0.9267 & $<2.2 \mathrm{e}-16$ \\
\hline & Cigarettes: THC: CBG: Binge.Alcohol & $480.252(250.09,710.41)$ & 4.32E-05 & lambda & -0.2760 & 0.0039 \\
\hline & Cigarettes: CBG & $339.558(175.8,503.32)$ & 4.82E-05 & & & \\
\hline & Binge.Alcohol & $1561.587(780.78,2342.4)$ & $8.86 \mathrm{E}-05$ & & & \\
\hline & CBG: Binge.Alcohol & $276.267(124.22,428.31)$ & 0.0004 & & & \\
\hline & Cigarettes: CBG: Binge.Alcohol & $-1470.381(-2232.93,-707.83)$ & 0.0002 & & & \\
\hline & CBG & $-63.136(-95.55,-30.73)$ & 0.0001 & & & \\
\hline & Cigarettes: THC: CBG & $-109.577(-164.86,-54.3)$ & 0.0001 & & & \\
\hline & Cigarettes: Binge.Alcohol & $-7753.892(-11,552.85,-3954.94)$ & $6.32 \mathrm{E}-05$ & & & \\
\hline & THC: Binge.Alcohol & $-796.23(-1149.18,-443.28)$ & $9.79 \mathrm{E}-06$ & & & \\
\hline & Cigarettes:THC & $-1143.639(-1586.1,-701.18)$ & 4.06E-07 & & & \\
\hline & 1 Years Lag & & & & & \\
\hline & Interactive Model-Cannabinoids & & & & & \\
\hline & spreml(Rate $\sim$ Cigarettes ${ }^{*} \mathrm{THC} * \mathrm{CB}$ & ${ }^{*} C B D+$ anlyr + Binge.Alcohol $+C o$ & aine) & & & \\
\hline THC, 1 & Cigarettes: CBD & $510(212.08,807.92)$ & 0.0008 & S.D. & 0.4457 & \\
\hline CBG, 1 & Cigarettes: THC: CBD & $563(229.8,896.2)$ & 0.0009 & LogLik & -91.2983 & \\
\hline \multirow[t]{8}{*}{$\mathrm{CBD}, 1$} & Cigarettes: THC: CBG & $1770(513.64,3026.36)$ & 0.0056 & psi & 0.8824 & $<2.2 \mathrm{e}-16$ \\
\hline & $\mathrm{THC}$ & $5.51(0.37,10.65)$ & 0.0356 & lambda & -0.3009 & 0.0050 \\
\hline & Cigarettes:THC & $-25.5(-50.78,-0.22)$ & 0.0479 & & & \\
\hline & THC: CBG & $-367(-625.72,-108.28)$ & 0.0054 & & & \\
\hline & Cigarettes: CBG: CBD & $-13,800(-22,286.8,-5313.2)$ & 0.0014 & & & \\
\hline & 2 Years Lag & & & & & \\
\hline & Interactive Model - Cannabinoids & & & & & \\
\hline & \multicolumn{6}{|c|}{ spreml(Rate $\sim$ Cigarettes ${ }^{*} T H C * C B G * C B D+$ anlyr + Binge.Alcohol + Cocaine) } \\
\hline THC, 2 & Cigarettes: CBG & $2040.99(821.21,3260.77)$ & 0.0010 & S.D. & 0.4457 & \\
\hline $\mathrm{CBG}, 2$ & CBG: CBD & $6381.11(2226.34,10,535.89)$ & 0.0026 & LogLik & -91.2983 & \\
\hline \multirow[t]{9}{*}{$\mathrm{CBD}, 2$} & $\mathrm{THC}$ & $10.36(1.06,19.65)$ & 0.0289 & psi & 0.8779 & $<2.2 \mathrm{e}-16$ \\
\hline & Cigarettes: THC & $-44.97(-88.58,-1.36)$ & 0.0432 & lambda & -0.4332 & 0.0001 \\
\hline & THC: CBG: CBD & $-4896.22(-8596.78,-1195.65)$ & 0.0095 & & & \\
\hline & $\mathrm{CBD}$ & $-177.12(-308.63,-45.6)$ & 0.0083 & & & \\
\hline & Cigarettes & $-70.36(-115.93,-24.79)$ & 0.0025 & & & \\
\hline & CBG & $-493.37(-753.84,-232.89)$ & 0.0002 & & & \\
\hline & 3 Years Lag & & & & & \\
\hline & Interactive Model - Cannabinoids & & & & & \\
\hline & \multicolumn{3}{|c|}{ spreml(Rate $\sim$ Cigarettes $* T H C{ }^{*} C B G * C B D+$ anlyr + Binge.Alcohol + Cocaine $)$} & S.D. & 0.4457 & \\
\hline THC, 3 & $\mathrm{CBD}$ & $3.38(0.51,6.26)$ & 0.0211 & LogLik & -91.2983 & \\
\hline$C B G, 3$ & Cigarettes: CBD & $-16.7(-29.52,-3.87)$ & 0.0107 & psi & 0.8615 & $<2.2 \mathrm{e}-16$ \\
\hline $\mathrm{CBD}, 3$ & Cigarettes & $-72.4(-122.84,-21.96)$ & 0.0049 & lambda & -0.3782 & 0.0162 \\
\hline
\end{tabular}


Table 17 Small Intestinal Stenosis or Atresia - Comprehensive Cannabinoid Space - Time Regression Models

\begin{tabular}{|c|c|c|c|c|c|c|}
\hline \multirow[t]{2}{*}{ Lagged Variables } & \multicolumn{3}{|l|}{ Parameter } & \multicolumn{3}{|c|}{ Model Parameters } \\
\hline & Parameter & Estimate (C.I.) & $P$-Value & Parameter & Value & Parameter $P$-Value \\
\hline & \multicolumn{6}{|c|}{ Interactive Model - Including Sociodemographics } \\
\hline & \multicolumn{6}{|c|}{$\begin{array}{l}\text { spreml(Rate } \sim \text { Cigarettes * } T H C * \text { CBG * CBD }+ \text { anlyr }+ \text { Binge.Alcohol + Cocaine + Income }+5 \\
\text { Races) }\end{array}$} \\
\hline & CBG & $1.15(0.45,1.85)$ & 0.0014 & S.D. & 0.4457 & \\
\hline & Cigarettes: CBD & $1.33(0.36,2.3)$ & 0.0071 & LogLik & -91.2983 & \\
\hline & Binge.Alcohol & $7.21(0.37,14.04)$ & 0.0388 & psi & 0.9046 & $<2.2 \mathrm{e}-16$ \\
\hline & $\mathrm{THC}$ & $-0.98(-1.96,-0.01)$ & 0.0476 & rho & -0.2587 & 0.01168 \\
\hline & \multicolumn{6}{|l|}{1 Years Lag } \\
\hline & \multicolumn{6}{|c|}{ Interactive Model - Including Sociodemographics } \\
\hline & \multicolumn{6}{|c|}{$\begin{array}{l}\text { spreml(Rate } \sim \text { Cigarettes * } T H C * \text { CBG * CBD + anlyr + Binge.Alcohol + Cocaine + Income }+5 \text { _ } \\
\text { Races) }\end{array}$} \\
\hline THC, 1 & Cigarettes: THC & $109.89(22.86,196.92)$ & 0.0133 & S.D. & 0.4457 & \\
\hline CBG, 1 & Cigarettes: THC: CBD & $24.48(3.55,45.41)$ & 0.0219 & LogLik & -91.2983 & \\
\hline \multirow[t]{6}{*}{$\mathrm{CBD}, 1$} & $\mathrm{THC}$ & $-22.12(-42.11,-2.14)$ & 0.0300 & psi & 0.8695 & $<2.2 \mathrm{e}-16$ \\
\hline & THC: CBD & $-5.4(-10.23,-0.58)$ & 0.0282 & rho & -0.3226 & 0.005233 \\
\hline & CBG & $-1.41(-2.34,-0.48)$ & 0.0030 & & & \\
\hline & \multicolumn{6}{|l|}{2 Years Lag } \\
\hline & \multicolumn{6}{|c|}{ Interactive Model - Including Sociodemographics } \\
\hline & \multicolumn{6}{|c|}{$\begin{array}{l}\text { spreml(Rate } \sim \text { Cigarettes * THC *CBG * CBD + anlyr + Binge.Alcohol + Cocaine + Income }+5 \text { _ } \\
\text { Races) }\end{array}$} \\
\hline THC, 2 & CBD & $1(0.41,1.6)$ & 0.0009 & S.D. & 0.4457 & \\
\hline $\mathrm{CBG}, 2$ & CBG & $1.74(0.53,2.94)$ & 0.0046 & LogLik & -91.2983 & \\
\hline \multirow[t]{2}{*}{ CBD, 2} & THC: CBD & $1.75(0.48,3.03)$ & 0.0072 & psi & 0.8514 & $<2.2 \mathrm{e}-16$ \\
\hline & $\mathrm{THC}$ & $5.8(0.8,10.8)$ & 0.0231 & rho & -0.4179 & 0.00155 \\
\hline
\end{tabular}

in the laboratory and across diverse human communities (in USA as a whole and in Hawaii, Colorado, Canada and Australia [13, 17-20]) implies that a much greater incidence of clinical teratogenesis might reasonably be expected to accompany this increased use, as was indeed recently demonstrated nationwide in USA for atrial septal defect secundum type [16] and for autism [66, 88] and has also recently been demonstrated in Canada and Australia [17-19]. This was also recently confirmed for all five chromsomal disorders reported across USA [89].

The present report is preliminary in the sense that a wider detailed geotemporospatial and causal inference study of many other congenital anomalies is clearly indicated. At the time of writing this more comprehensive and detailed manuscript is in preparation. Our unpublished findings are that such upper range predicted curve positive inflections and sigmoidality are typical and normative amongst geospatial models for almost all positively impacted congenital anomalies studied to date. Also strongly indicated are geotemporospatial studies at finer geospatial resolution such as was recently published from CDC for gastroschisis at county level and which employed similar prevalence ratio methodology to the present study [90].

One notes also that the USA is moving relatively rapidly into an era when cannabinoids are more widely available than previously as the legislative regimes relating to cannabis are progressively relaxed. The replacement of tobacco crops in many places with hemp crops implies that cannabinoids of various forms will increasingly enter the food chain both explicitly as lollies, candies, chocolates, sauces, health foods and oils, and implicitly as stock feed, bird feed and in dairy and egg products. It therefore seems inevitable in such a paradigm that population level cannabinoid exposure will necessarily increase. In this context the traditional way of doing teratological studies by simply asking a binary question as to maternal antenatal exposure to cannabis becomes increasingly inaccurate and passé. Calls for a quantitative biomarker of cannabinoid exposure have been issued derived potentially from epigenomic and / or glycomic metrics [91]. As we enter an era of more widespread known and unknown cannabinoid exposure in the community, higher level cannabinoid potency, 
Table 18 Small Intestinal Stenosis or Atresia - E-Values from Mixed Effects and Panel Regression Models

\begin{tabular}{|c|c|c|c|}
\hline Parameter & Estimate (C.I.) & R.R. (C.I.) & E-Values \\
\hline \multicolumn{4}{|l|}{ MIXED EFFECTS } \\
\hline \multicolumn{4}{|l|}{ Cannabis Only } \\
\hline Cannabis & $2.83(2.03,3.63)$ & $5.66(3.48,3.19)$ & $10.80,6.43$ \\
\hline \multicolumn{4}{|l|}{ Additive Model - Drugs } \\
\hline Cannabis & $1.46(0.69,2.22)$ & $3.91(1.93,7.92)$ & $7.28,3.27$ \\
\hline \multicolumn{4}{|l|}{ Interactive Model-Drugs } \\
\hline Cigarettes: Cannabis: Binge.Alcohol & $5638.66(3549.85,7727.46)$ & Infinity (Infinity, Infinity) & Infinity, Infinity \\
\hline Cigarettes: Cannabis: Binge.Alcohol: Analgesics & $1797.36(1122.19,2472.54)$ & Infinity (Infinity, Infinity) & Infinity, Infinity \\
\hline Cannabis: Binge.Alcohol & $8008.91(4682.06,11,335.77)$ & Infinity (Infinity, Infinity) & Infinity, Infinity \\
\hline Cannabis: Binge.Alcohol: Analgesics & $2546(1467.93,3624.06)$ & Infinity (Infinity, Infinity) & Infinity, Infinity \\
\hline \multicolumn{4}{|l|}{ Additive Model-Cannabinoids } \\
\hline THC & $0.94(0.41,1.48)$ & $2.41(1.47,3.93)$ & $4.24,2.31$ \\
\hline CBD & $0.84(0.25,1.43)$ & $2.18(1.27,3.74)$ & $3.78,1.85$ \\
\hline \multicolumn{4}{|l|}{ Interactive Model - Cannabinoids } \\
\hline CBG: CBD & $10.47(7.47,13.47)$ & $4.59 \mathrm{E}+06(6.01 \mathrm{E}+04,3.51 \mathrm{E}+08)$ & $3.18 \mathrm{E}+06,1.20 \mathrm{E}+05$ \\
\hline CBD & $34.98(24.72,45.24)$ & $1.85 \mathrm{E}+22(6.74 \mathrm{E}+15,5.11 \mathrm{E}+28)$ & $3.71 \mathrm{E}+22,1.34 \mathrm{E}+16$ \\
\hline THC: CBG: CBD & $0.57(0.37,0.76)$ & $2.29(1.73,3.04)$ & $4.03,2.87$ \\
\hline CBG & $32.45(19.49,45.41)$ & $2.45 \mathrm{E}+20(3.32 \mathrm{E}+12,6.15 \mathrm{E}+28)$ & $3.05 E+20,6.65 E+12$ \\
\hline Cigarettes: THC: CBD & $13.79(6.1,21.48)$ & $6.03 \mathrm{E}+08(8.99 \mathrm{E}+06,4.03 \mathrm{E}+13)$ & $1.21 \mathrm{E}+09,1.80 \mathrm{E}+04$ \\
\hline \multicolumn{4}{|l|}{ Additive Model - Including Sociodemographics } \\
\hline THC & $1.45(0.79,2.12)$ & $3.97(2.12,7.41)$ & $7.41,3.67$ \\
\hline $\mathrm{CBD}$ & $0.81(0.21,1.4)$ & $2.145(1.24,3.77)$ & $3.74,1.77$ \\
\hline \multicolumn{4}{|l|}{ Interactive Model - Including Sociodemographics } \\
\hline CBG & $77.88(58.11,97.66)$ & $3.15 E+38(7.30 E+28,1.36 E+48)$ & $6.30 \mathrm{E}+38,1.46 \mathrm{E}+29$ \\
\hline CBD & $63.63(47.13,80.13)$ & $2.82 E+31(2.57 E+23,3.09 E+39)$ & $5.64 \mathrm{E}+31,5.15 \mathrm{E}+23$ \\
\hline CBG: CBD & $18.44(13.62,23.25)$ & $1.29 \mathrm{E}+09(5.85 \mathrm{E}+06,2.87 \mathrm{E}+11)$ & $2.59 \mathrm{E}+09,1.17 \mathrm{E}+07$ \\
\hline Cigarettes: THC & $2351.21(1186.17,3516.25)$ & Infinity (Infinity, Infinity) & Infinity, Infinity \\
\hline Cigarettes: THC: CBD & $548.39(275.16,821.63)$ & 2.07E+284 (4.31E+141, Infinity) & Infinity, $8.61 E+141$ \\
\hline Cigarettes: THC: CBG: CBD & $135.08(66.39,203.77)$ & $5.92 \mathrm{E}+66(2.01 \mathrm{E}+33,1.74 \mathrm{E}+100)$ & $1.18 \mathrm{E}+67,4.03 \mathrm{E}+33$ \\
\hline Cigarettes: THC: CBG & $575.97(282.33,869.61)$ & $1.19 E+271(8.89 E+137$, Infinity) & Infinity, 1.77E+138 \\
\hline \multicolumn{4}{|l|}{ PANEL MODELS } \\
\hline \multicolumn{4}{|l|}{ Additive Model - Including Sociodemographics } \\
\hline CBG & $1.07(0.51,1.63)$ & $3.31(1.77,3.17)$ & $6.06,2.94$ \\
\hline CBD & $0.61(0.23,0.99)$ & $1.97(1.298,3.02)$ & $3.36,1.91$ \\
\hline \multicolumn{4}{|l|}{ Interactive Model - Including Sociodemographics } \\
\hline Cigarettes: THC & $20.18(10.52,29.83)$ & $4.31 \mathrm{E}+28(9.13 \mathrm{E}+14,2.04 \mathrm{E}+42)$ & $8.63 \mathrm{E}+28,1.82 \mathrm{E}+15$ \\
\hline CBG: CBD & $0.92(0.4,1.44)$ & $20.014(3.65,109.74)$ & $39.53,6.76$ \\
\hline $\mathrm{CBD}$ & $3.68(1.19,6.16)$ & $1.65 \mathrm{E}+05(49.84,5.46 \mathrm{E}+08)$ & $3.30 \mathrm{E}+05,99.18$ \\
\hline \multicolumn{4}{|l|}{1 Years Lag } \\
\hline Cigarettes: THC & $6.68(3.64,9.72)$ & $2.42 \mathrm{E}+06(70.07,8.34 \mathrm{E}+03)$ & $4.83 E+03,139.63$ \\
\hline
\end{tabular}

higher intensity cannabis use and the widespread availability of highly concentrated cannabinoid oils, dabs, waxes, shatters, extracts and products it seems that the urgency of deriving such a quantitative biomarker necessarily proportionately increases. An important corollary of the deployment of such an objective biomarker is that much smaller numbers of maternal-foetal pairs can be used to measure effect sizes and the chance of misattribution is potentially greatly reduced with the added advantage for analysis and for statistical power that cannabinoid exposure can be treated more properly as a continuous variable. 
Table 19 Small Intestinal Stenosis or Atresia - E-Values from Space - Time Regression Models

\begin{tabular}{|c|c|c|c|}
\hline Parameter & Estimate (C.I.) & R.R. (C.I.) & E-Values \\
\hline \multicolumn{4}{|l|}{ SPACE-TIME MODELS } \\
\hline \multicolumn{4}{|l|}{ Additive Model - Drugs } \\
\hline Cannabis & $1.15(0.46,1.84)$ & $9.60(2.48,37.17)$ & $18.70,4.40$ \\
\hline \multicolumn{4}{|l|}{ Interactive Model - Drugs } \\
\hline Cigarettes: Cannabis: Binge.Alcohol & $57.95(30.14,85.75)$ & $2.40 \mathrm{E}+28(6.17 \mathrm{E}+14,9.36 \mathrm{E}+41)$ & $4.81 \mathrm{E}+28,1.23 \mathrm{E}+15$ \\
\hline Cannabis: Binge.Alcohol & $30.95(15.37,46.53)$ & 1.44E+15 (3.48E+07, 5.96E+22) & $2.88 \mathrm{E}+15,6.96 \mathrm{E}+07$ \\
\hline Cigarettes: Cannabis: Binge.Alcohol: Analgesics & $11.55(3.04,20.06)$ & $4.54 \mathrm{E}+05(31.57,6.55 \mathrm{E}+09)$ & $9.09 \mathrm{E}+05,62.64$ \\
\hline \multicolumn{4}{|l|}{2 Years Lag } \\
\hline \multicolumn{4}{|l|}{ Interactive Model - Drugs } \\
\hline Cannabis: Analgesics & $68.51(39.94,97.07)$ & $6.69 \mathrm{E}+62(4.80 \mathrm{E}+36,9.34 \mathrm{E}+88)$ & $1.33 \mathrm{E}+63,9.61 \mathrm{E}+36$ \\
\hline \multicolumn{4}{|l|}{4 Years Lag } \\
\hline \multicolumn{4}{|l|}{ Interactive Model-Drugs } \\
\hline Cannabis: Analgesics & $1284.76(677.88,1891.64)$ & Infinity (Infinity, Infinity) & Infinity, Infinity \\
\hline Cannabis & $4106.59(2160.15,6053.02)$ & Infinity (Infinity, Infinity) & Infinity, Infinity \\
\hline \multicolumn{4}{|l|}{ Additive Model - Cannabinoids } \\
\hline CBG & $0.96221(0.28,1.64)$ & $16.09(2.45,105.29)$ & $31.67,4.35$ \\
\hline \multicolumn{4}{|l|}{ Interactive Model-Cannabinoids } \\
\hline Cigarettes:THC: Binge.Alcohol & $5169.433(3191.79,7147.08)$ & Infinity (Infinity, Infinity) & Infinity, Infinity \\
\hline $\mathrm{THC}$ & $172.247(93.57,250.92)$ & $4.79 E+103(2.62 E+56,8.74 E+150)$ & $9.58 \mathrm{E}+103,5.25 \mathrm{E}+56$ \\
\hline Cigarettes: THC: CBG: Binge.Alcohol & $480.252(250.09,710.41)$ & $1.19 \mathrm{E}+289(6.58 \mathrm{E}+150$, Infinity $)$ & Infinity, 1.31E+151 \\
\hline Cigarettes: CBG & $339.558(175.8,503.32)$ & $2.45 E+204(1.04 E+106,5.80 E+302)$ & Infinity, 2.08E+106 \\
\hline CBG: Binge.Alcohol & $276.267(124.22,428.31)$ & $1.96 \mathrm{E}+166(9.07 \mathrm{E}+74,4.26 \mathrm{E}+257)$ & Infinity, 1.81E+75 \\
\hline \multicolumn{4}{|l|}{1 Years Lag } \\
\hline \multicolumn{4}{|l|}{ Interactive Model-Cannabinoids } \\
\hline Cigarettes: CBD & $510(212.08,807.92)$ & Infinity (2.65E+187, Infinity) & Infinity, Infinity \\
\hline Cigarettes: THC: CBD & $563(229.8,896.2)$ & Infinity (1.78E+204, Infinity) & Infinity, Infinity \\
\hline Cigarettes: THC: CBG & $1770(513.64,3026.36)$ & Infinity (Infinity, Infinity) & Infinity, Infinity \\
\hline $\mathrm{THC}$ & $5.51(0.37,10.65)$ & 7.74E+04 $(2.18,2.74 \mathrm{E}+09)$ & $1.55 \mathrm{E}+05,3.79$ \\
\hline \multicolumn{4}{|l|}{2 Years Lag } \\
\hline \multicolumn{4}{|l|}{ Interactive Model - Cannabinoids } \\
\hline Cigarettes: CBG & $2040.99(821.21,3260.77)$ & Infinity (Infinity, Infinity) & Infinity, Infinity \\
\hline CBG: CBD & $6381.11(2226.34,10,535.89)$ & Infinity (Infinity, Infinity) & Infinity, Infinity \\
\hline THC & $10.36(1.06,19.65)$ & 7.65E+09 $(10.81,5.41 \mathrm{E}+18)$ & $1.53 \mathrm{E}+10,21.11$ \\
\hline \multicolumn{4}{|l|}{3 Years Lag } \\
\hline \multicolumn{4}{|l|}{ Interactive Model-Cannabinoids } \\
\hline CBD & $3.38(0.51,6.26)$ & $183.44(2.20,1.52 \mathrm{E}+04)$ & $366.39,3.83$ \\
\hline \multicolumn{4}{|l|}{ Interactive Model - Including Sociodemographics } \\
\hline CBG & $1.15(0.45,1.85)$ & $11.34(2.58,49.90)$ & $22.17,4.59$ \\
\hline Cigarettes: CBD & $1.33(0.36,2.3)$ & $16.55(2.15,127.21)$ & $32.59,3.72$ \\
\hline \multicolumn{4}{|l|}{1 Years Lag } \\
\hline \multicolumn{4}{|l|}{ Interactive Model - Including Sociodemographics } \\
\hline Cigarettes: THC & $109.89(22.86,196.92)$ & $4.32 \mathrm{E}+91(1.62 \mathrm{E}+19,1.15 \mathrm{E}+164)$ & $8.68 \mathrm{E}+91,3.25 \mathrm{E}+19$ \\
\hline Cigarettes: THC: CBD & $24.48(3.55,45.41)$ & $2.57 \mathrm{E}+20(985.96,6.70 \mathrm{E}+37)$ & $5.14 \mathrm{E}+20,1.97 \mathrm{E}+03$ \\
\hline \multicolumn{4}{|l|}{2 Years Lag } \\
\hline \multicolumn{4}{|l|}{ Interactive Model - Including Sociodemographics } \\
\hline $\mathrm{CBD}$ & $1.00(0.41,1.60)$ & $6.70(2.18,20.54)$ & $12.89,3.80$ \\
\hline CBG & $1.74(0.53,2.94)$ & $26.83(2.76,260.21)$ & $53.17,4.98$ \\
\hline THC: CBD & $1.75(0.48,3.03)$ & $274.86(2.47,313.56)$ & $55.22,4.38$ \\
\hline THC & $5.80(0.80,10.8)$ & $2.96 \mathrm{E}+04(4.60,7.71 \mathrm{E}+08)$ & 1.19E+05, 6.68 \\
\hline
\end{tabular}



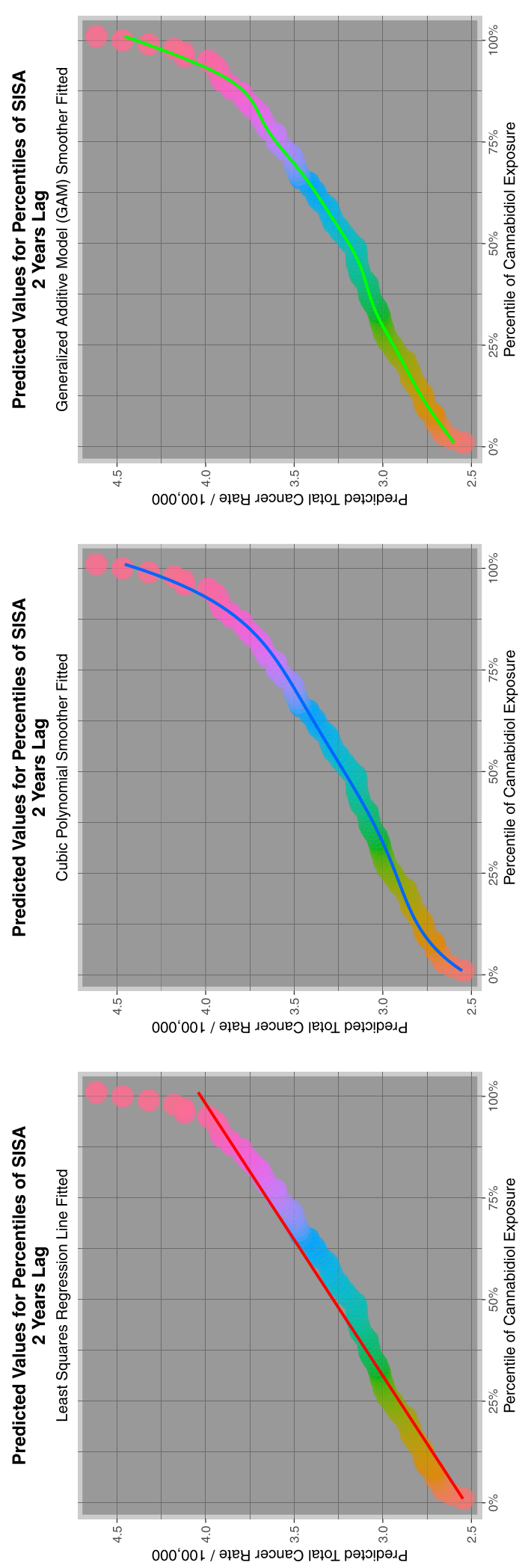

Fig. 17 Modelled rates of small intestinal stenosis or atresia rates with rising cannabidiol exposure in a geospatial model lagged to two years

\section{Mechanistic considerations \\ Role of morphogen gradients in body pattern formation}

The gradients of various key morphogens control of the formation of the body in many respects [92]. This is well illustrated in the case of the neural tube which goes to form the spinal cord and central nervous system. Bone morphogenetic proteins and Wnts are released from the dorsal roof plate region in high concentration. Sonic hedgehog (shh) is released form the notochord and induces shh release form the ventral floorplate of the neural tube in high concentration [92]. Hence between the dorsal roof plate and the ventral floor plate there exist opposing and antagonistic gradients from BMPs and Wnts dorsally as against shh ventrally. Shh suppresses class I factors (Pax-3/7, Dbx-1, Dbx-2, Irx 3 and Pax-6) and stimulates class II factors (Foxa-2, Nkx-6.2, Nkx-6.1, Olig-2, Nkx-2.2 and Nkx-2.9). These opposing gradients specify in detail the nature of the neurons which will develop in the various loci of the developing neural tube. At the same time lateral gradients of retinoic acid emanate from the lateral edges of the neural tube descending to very low concentrations along the lumen of the neural tube. Rostral-causal axial differentiation is controlled by opposing gradients of retinoic acid rostrally competing with FGF and Gli1 from the caudal end of the neural tube [92].

Hence in a very real way one could say that the structures of the neural tube are actually woven together by opposing and antagonistic but balanced morphogen gradients. Similar principles often operate in numerous other tissues at the level of the overall body pattern, at the organ level, for body rotation where it is not symmetrical, and at the cellular and subcellular levels.

In considering the impacts of cannabinoids on the forming embryo it is of interest to consider the effects cannabinoids might have on one of the main morphogen systems in the body which is sonic hedgehog. A brief consideration of their impacts on other fundamental morphogen systems follows.

\section{Sonic Hedgehog}

Sonic Hedgehog (shh) is one of the most important of all the body morphogens. Indeed one contemporary textbook includes 174 references to this key morphogen [92]. 

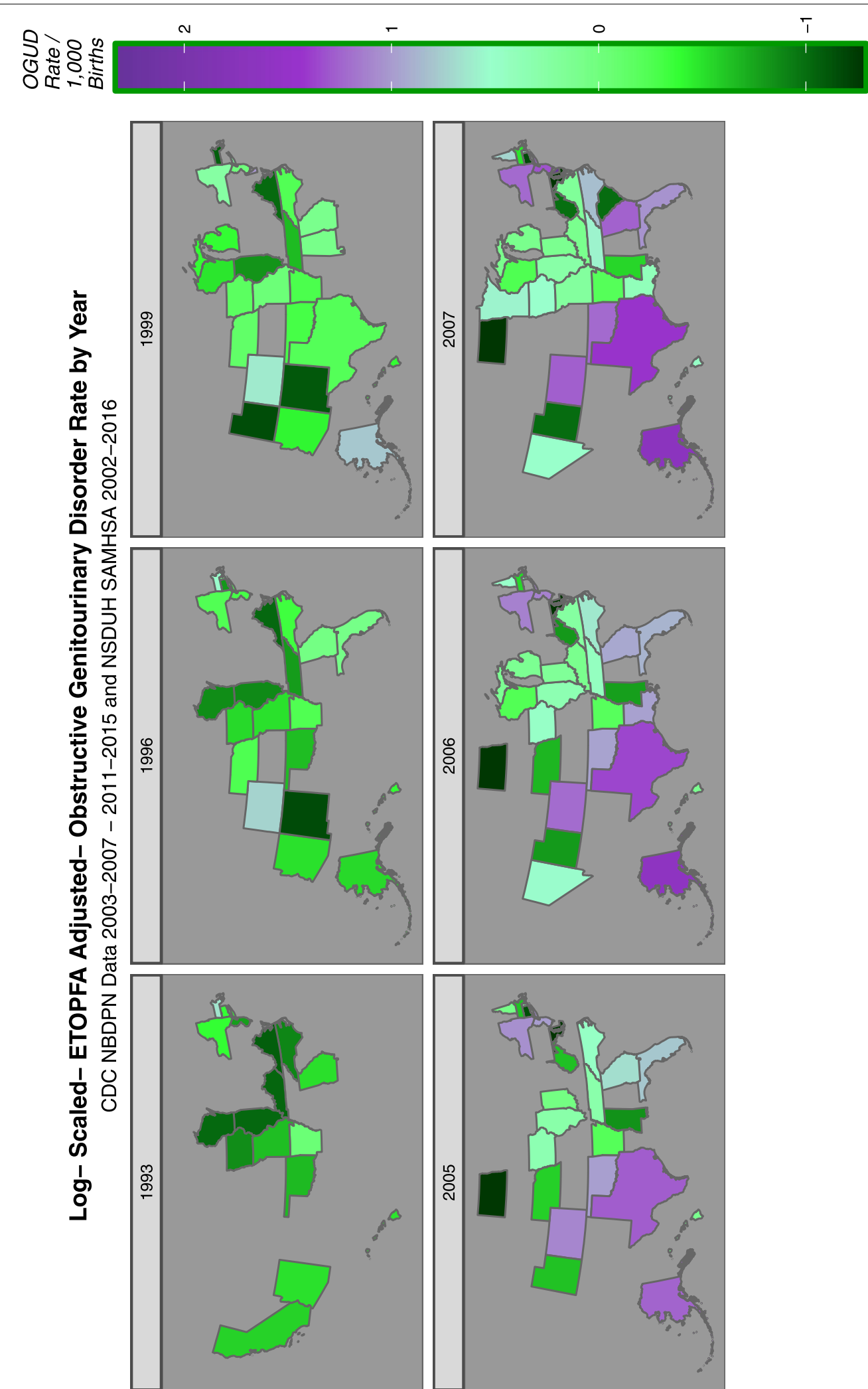

Fig. 18 Map-graph of the incidence of obstructive genitourinary defects across USA over time 
Table 20 Obstructive Genitourinary Defects - Introductory Space - Time Regression Models

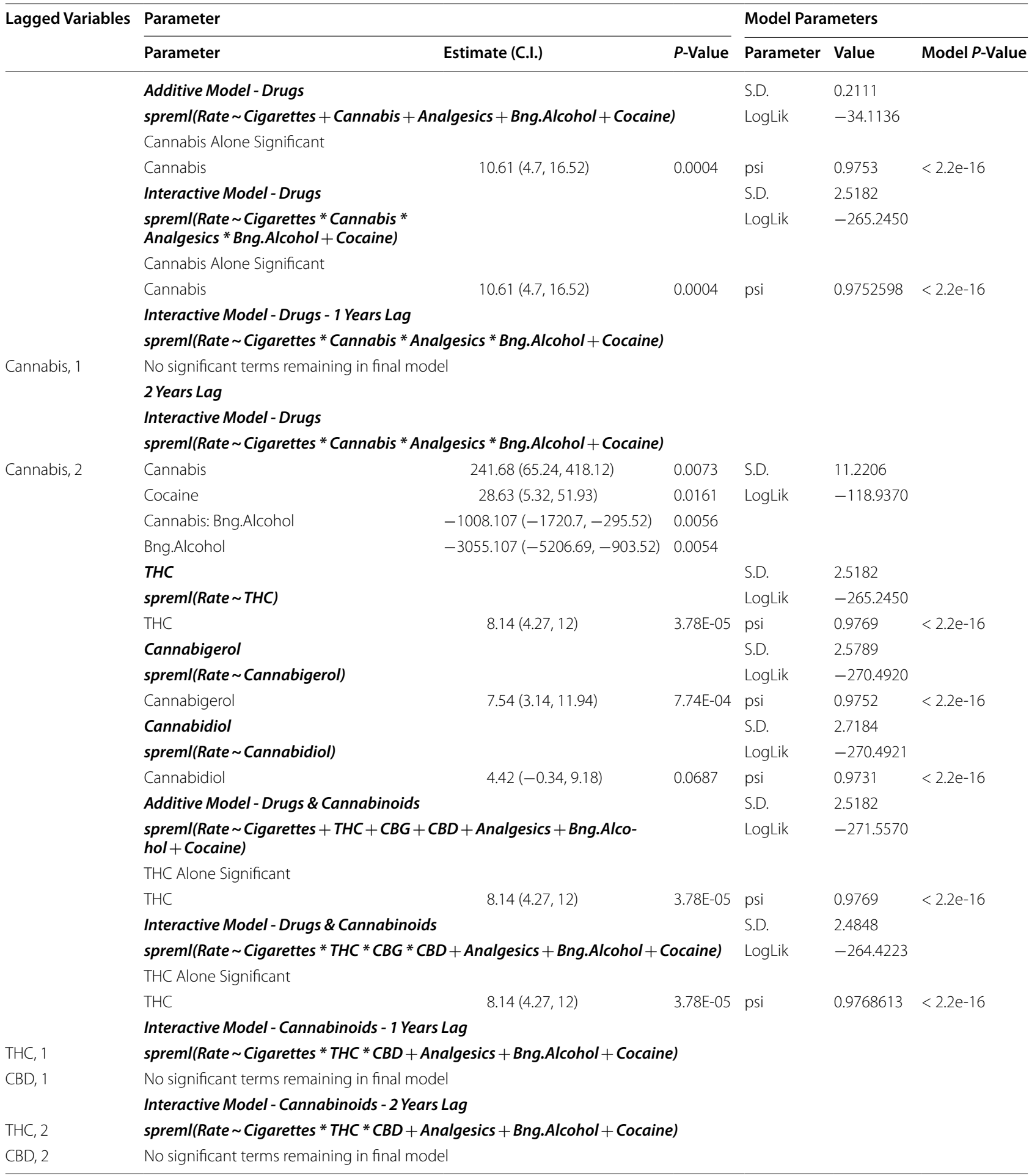


Table 21 Obstructive Genitourinary Defects - Cannabinoid Space - Time Regression Models

\begin{tabular}{|c|c|c|c|c|c|c|}
\hline \multirow[t]{2}{*}{ Lagged Variables } & \multicolumn{3}{|l|}{ Parameter } & \multicolumn{3}{|c|}{ Model Parameters } \\
\hline & Parameter & Estimate (C.I.) & $P$-Value & Parameter & Value & Model $P$-Value \\
\hline & \multicolumn{6}{|c|}{1 Spatial Lag - Interactive Model, Cannabinoids } \\
\hline & \multicolumn{6}{|l|}{$T H C * C B D$} \\
\hline & \multicolumn{6}{|c|}{ spreml(Rate $\sim$ Cigarettes * $T H C *$ CBD + Analgesics + Bng.Alcohol + Cocaine $)$} \\
\hline $\mathrm{THC}, 2$ & Cigarettes & $2767.39(1031.71,4503.07)$ & 0.0018 & S.D. & 2.4975 & \\
\hline \multirow[t]{9}{*}{$\mathrm{CBD}, 2$} & Cigarettes: CBD & $792.04(292.87,1291.2)$ & 0.0019 & LogLik & -264.8543 & \\
\hline & Cigarettes: THC: CBD & $912.27(282.87,1541.68)$ & 0.0045 & psi & 0.9768 & $<2.2 \mathrm{e}-16$ \\
\hline & Cigarettes: THC & $3167.74(941.61,5393.88)$ & 0.0053 & & & \\
\hline & THC & $-712.92(-1234.34,-191.5)$ & 0.0074 & & & \\
\hline & THC: CBD & $-208.76(-356.95,-60.56)$ & 0.0058 & & & \\
\hline & CBD & $-188.38(-305.1,-71.66)$ & 0.0016 & & & \\
\hline & \multicolumn{6}{|c|}{1 Spatial Lag - Interactive Model, Cannabinoids } \\
\hline & \multicolumn{6}{|c|}{$T H C * C B G$} \\
\hline & \multicolumn{6}{|c|}{ spreml(Rate $\sim$ Cigarettes ${ }^{*} T H C * C B G+$ Analgesics + Bng.Alcohol + Cocaine) } \\
\hline $\mathrm{THC}, 2$ & Cigarettes: THC: CBG & $855.74(286.28,1425.2)$ & 0.0032 & S.D. & 2.4975 & \\
\hline \multirow[t]{8}{*}{$\mathrm{CBG}, 2$} & Cigarettes: THC & $2980.74(841.4,5120.08)$ & 0.0063 & LogLik & -264.8543 & \\
\hline & Cigarettes & $2664.14(705.35,4622.92)$ & 0.0077 & psi & 0.9768 & $<2.2 \mathrm{e}-16$ \\
\hline & Cigarettes: CBG & $755.71(171.07,1340.34)$ & 0.0113 & & & \\
\hline & THC & $-655.51(-1157.24,-153.79)$ & 0.0104 & & & \\
\hline & CBG & $-185.75(-327.89,-43.62)$ & 0.0104 & & & \\
\hline & THC: CBG & $-194.05(-327.64,-60.46)$ & 0.0044 & & & \\
\hline & \multicolumn{6}{|c|}{1 Spatial, 1 Temporal Lag - Interactive Model, Cannabinoids } \\
\hline & \multicolumn{6}{|c|}{ spreml(Rate $\sim$ Cigarettes ${ }^{*} T H C * C B D+$ Analgesics + Bng.Alcohol+ Cocaine $)$} \\
\hline THC, 1 & Cigarettes: THC: CBD & $1394.48(386.59,2402.38)$ & 0.0067 & S.D. & 2.8611 & \\
\hline $\mathrm{CBD}, 1$ & Cigarettes: THC: THC.Spatial: CBD & $1384.11(374.49,2393.72)$ & 0.0072 & LogLik & -189.0979 & \\
\hline \multirow[t]{11}{*}{ THC, 1 Spatial } & Cigarettes: THC & $5000.58(1323.37,8677.78)$ & 0.0077 & psi & 0.9833 & $<2.2 \mathrm{e}-16$ \\
\hline & Cigarettes: THC: THC.Spatial & $4975.93(1182.67,8769.19)$ & 0.0101 & & & \\
\hline & Cigarettes & $1787.24(184.11,3390.37)$ & 0.0289 & & & \\
\hline & Cigarettes: CBD & $522.98(44.27,1001.7)$ & 0.0323 & & & \\
\hline & CBD & $-134.19(-253.84,-14.55)$ & 0.0279 & & & \\
\hline & THC & $-1084.97(-1955.48,-214.47)$ & 0.0146 & & & \\
\hline & THC:THC & $-1084.66(-1951.65,-217.67)$ & 0.0142 & & & \\
\hline & THC: CBD & $-311.84(-552.33,-71.36)$ & 0.0110 & & & \\
\hline & THC: THC: CBD & $-307.33(-537.59,-77.07)$ & 0.0089 & & & \\
\hline & \multicolumn{6}{|c|}{1 Spatial, 2 Temporal Lags - Interactive Model, Cannabinoids } \\
\hline & \multicolumn{6}{|c|}{ spreml(Rate Cigarettes *THC *CBD+Analgesics + Bng.Alcohol+ Cocaine) } \\
\hline THC, 2 & Cigarettes & $137,535.9(58,078.87,216,992.93)$ & 0.0007 & S.D. & 9.6638 & \\
\hline $\mathrm{CBD}, 2$ & Cigarettes: CBD & $48,350.5(20,095.92,76,605.08)$ & 0.0008 & LogLik & -116.844 & \\
\hline \multirow[t]{9}{*}{ THC, 1 Spatial } & Cigarettes: THC & $217,699.3(89,605.46,345,793.14)$ & 0.0009 & rho & -0.68203 & 0.002462 \\
\hline & Cigarettes: THC: CBD & $76,973.5(31,232.59,122,714.41)$ & 0.0010 & & & \\
\hline & THC & $11,707.8(4631.81,18,783.79)$ & 0.0012 & & & \\
\hline & THC:THC.Spatial & $19,063(7395.32,30,730.68)$ & 0.0014 & & & \\
\hline & THC: CBD & $-18,888.3(-30,246.11,-7530.49)$ & 0.0011 & & & \\
\hline & Cigarettes: THC:THC & $-78,290.4(-125,332.16,-31,248.64)$ & 0.0011 & & & \\
\hline & THC & $-53,462(-85,264.37,-21,659.63)$ & 0.0010 & & & \\
\hline & Cigarettes: THC & $-48,251.3(-76,830.65,-19,671.95)$ & 0.0009 & & & \\
\hline & CBD & $-11,798.1(-18,785.3,-4810.9)$ & 0.0009 & & & \\
\hline
\end{tabular}


Table 22 Obstructive Genitourinary Defects - Comprehensive Cannabinoid Space - Time Regression Models

\begin{tabular}{|c|c|c|c|c|c|c|}
\hline \multirow[t]{2}{*}{ Lagged Variables } & \multicolumn{3}{|l|}{ Parameter } & \multicolumn{3}{|c|}{ Model Parameters } \\
\hline & Parameter & Estimate (C.I.) & $P$-Value & Parameter & Value & Model P-Value \\
\hline & \multirow{2}{*}{\multicolumn{6}{|c|}{$\begin{array}{l}\text { Interactive Model - Including Sociodemographics } \\
\text { spreml(Rate } \sim \text { Cigarettes * } T H C * C B D+\text { Analgesics }+ \text { Bng.Alcohol + Cocaine + Income }+5 \\
\text { Races) }\end{array}$}} \\
\hline & & & & & & \\
\hline & Hispanic & $7.56(3.56,11.55)$ & 0.0002 & S.D. & \multicolumn{2}{|l|}{2.3684} \\
\hline & $\mathrm{THC}$ & $37.58(9.36,65.79)$ & 0.0090 & LogLik & \multicolumn{2}{|l|}{-254.1933} \\
\hline & Am.Indian/Alaskan.Native & $124.12(30.78,217.46)$ & 0.0092 & psi & 0.9663 & $<2.2 \mathrm{e}-16$ \\
\hline & THC: CBG & $6.95(0.33,13.56)$ & 0.0395 & & & \\
\hline & Income & $-13.2(-23.45,-2.94)$ & 0.0117 & & & \\
\hline & \multicolumn{6}{|l|}{1 Years Lag } \\
\hline & \multicolumn{6}{|c|}{ Interactive Model - Including Sociodemographics } \\
\hline & \multicolumn{6}{|c|}{$\begin{array}{l}\text { spreml(Rate } \sim \text { Cigarettes * THC * CBD }+ \text { Analgesics }+ \text { Bng.Alcohol + Cocaine + Income }+5 \\
\text { Races) }\end{array}$} \\
\hline THC, 1 & Hispanic & $7.59(3.07,12.12)$ & 0.0010 & S.D. & 3.2724 & \\
\hline \multirow[t]{6}{*}{ CBD, 1} & Cigarettes: THC & $46.25(16.84,75.67)$ & 0.0021 & LogLik & -187.7251 & \\
\hline & Am.Indian/Alaskan.Native & $148.61(47.3,249.93)$ & 0.0040 & psi & 0.9689 & $<2.2 \mathrm{e}-16$ \\
\hline & Income & $-17.24(-30.36,-4.12)$ & 0.0100 & & & \\
\hline & \multicolumn{6}{|l|}{2 Years Lag } \\
\hline & \multicolumn{6}{|c|}{ Interactive Model - Including Sociodemographics } \\
\hline & \multicolumn{6}{|c|}{$\begin{array}{l}\text { spreml(Rate } \sim \text { Cigarettes * THC * CBD }+ \text { Analgesics }+ \text { Bng.Alcohol }+ \text { Cocaine }+ \text { Income }+5 \\
\text { Races) }\end{array}$} \\
\hline THC, 2 & Hispanic & $12.81(8.33,17.3)$ & 2.17E-08 & S.D. & 3.2724 & \\
\hline \multirow[t]{9}{*}{$C B D, 2$} & Cigarettes: THC: CBD & $6151.83(2693.75,9609.91)$ & 0.0005 & LogLik & -187.7251 & \\
\hline & Cigarettes: THC & $22,951.53(9883.29,36,019.77)$ & 0.0006 & psi & 0.0000 & NA \\
\hline & Cigarettes & $15,335.11(5177.59,25,492.63)$ & 0.0031 & & & \\
\hline & Cigarettes: CBD & $4078.6(1248.29,6908.9)$ & 0.0047 & & & \\
\hline & Am.Indian/Alaskan.Native & $107.64(18.06,197.22)$ & 0.0185 & & & \\
\hline & $\mathrm{CBD}$ & $-894.76(-1549.98,-239.53)$ & 0.0074 & & & \\
\hline & Bng.Alcohol & $-186.96(-318.68,-55.23)$ & 0.0054 & & & \\
\hline & THC & $-5115.33(-8111.04,-2119.62)$ & 0.0008 & & & \\
\hline & THC: CBD & $-1370.14(-2158.79,-581.48)$ & 0.0007 & & & \\
\hline
\end{tabular}

Shh has been shown to be critically involved in the development of the following structures [92]:

Gastrula / Early Embryo

- Primitive node of the late gastrula

- Notochord

- shh gradient along ventral surface of embryo

- Gradient antagonizes its opposing morphogens, particularly FGFs, from posterior embryo

Brain

- Early Forebrain specifier and organizer

- Controls ventral midbrain formation including the ventral tegmental area and Nucleus Accumbens
- Cerebellum organizer - The large Purkinje cell secrete shh which stimulates granule cell proliferation [92]

- Induces motor neuron development in the ventral neural tube [92]

Face

- Face organizer [92]

- Shh is critical for the outgrowth of the Palatal shelves

- Ectodermal tips of the facial processes

- Controls midline tongue fusion

- Controls development of the filiform papillae on the tongue

- Controls tooth development

- Controls taste bud development

- Apical ectoderm of second pharyngeal pouch [92] 
Table 23 Obstructive Genitourinary Defects - E-Values from Mixed Effects and Panel Regression Models

\begin{tabular}{|c|c|c|c|}
\hline Parameter & Estimate (C.I.) & R.R. (C.I.) & E-Values \\
\hline \multicolumn{4}{|l|}{ MIXED EFFECTS MODELS } \\
\hline \multicolumn{4}{|l|}{ Cannabis Only } \\
\hline Cannabis & $14.35(8.44,20.27)$ & $94.85(15.13,594.66)$ & $189.20,29.75$ \\
\hline \multicolumn{4}{|l|}{ Additive Model - Drugs } \\
\hline Cannabis & $14.35(8.44,20.27)$ & $94.85(15.13,594.66)$ & $189.20,29.75$ \\
\hline \multicolumn{4}{|l|}{ Interactive Model - Drugs } \\
\hline Cigarettes: Cannabis: Analgesics & $333.48(176.14,490.83)$ & $1.62 \mathrm{E}+51(4.54 \mathrm{E}+27,5.81 \mathrm{E}+74)$ & $3.25 \mathrm{E}+51,9.09 \mathrm{E}+27$ \\
\hline Cannabis: Bng.Alcohol: Analgesics & $700.3(368.06,1032.54)$ & $3.47 \mathrm{E}+107(6.41 \mathrm{E}+57,1.88 \mathrm{E}+157)$ & $6.94 \mathrm{E}+107,1.29 \mathrm{E}+58$ \\
\hline Cannabis: Bng.Alcohol & $921.93(370.26,1473.6)$ & $3.75 \mathrm{E}+1241\left(9.87 \mathrm{E}+58,1.43 \mathrm{E} \_224\right)$ & $7.51 \mathrm{E}+141,1.97 \mathrm{E}+59$ \\
\hline \multicolumn{4}{|l|}{ Additive Model - Cannabinoids } \\
\hline THC & $43.47(8.06,78.89)$ & $1.72 \mathrm{E}+06(18.61,1.59 \mathrm{E}+11)$ & $3.43 E+06,36.72$ \\
\hline \multicolumn{4}{|l|}{ Interactive Model-Cannabinoids } \\
\hline Cigarettes: THC & $1945.4(832.31,3058.5)$ & $2.73 E+296(5.73 E+130$, Infinity $)$ & Infinity, 1.14E+131 \\
\hline Cigarettes: THC: CBG & $482.22(204.16,760.28)$ & $3.02 \mathrm{E}+73(1.23 \mathrm{E}+32,7.37 \mathrm{E}+114)$ & $6.04 \mathrm{E}+73,2.46 \mathrm{E}+32$ \\
\hline \multicolumn{4}{|c|}{ Additive Model - Including Sociodemographics } \\
\hline $\mathrm{THC}$ & $11.62(7.82,15.42)$ & $58.96(16.01,217.10)$ & $117.42,31.52$ \\
\hline \multicolumn{4}{|c|}{ Interactive Model - Including Sociodemographics } \\
\hline THC: CBG & $918.55(286.58,1550.52)$ & $2.27 \mathrm{E}+138(4.07 \mathrm{E}+45,1.27 \mathrm{E}+231)$ & $4.55 E+138,8.15 E+45$ \\
\hline THC: CBG: CBD & $248.54(72.69,424.4)$ & $2.73 \mathrm{E}+37(4.24 \mathrm{E}+11,1.76 \mathrm{E}+63)$ & $5.46 \mathrm{E}+37,8.49 \mathrm{E}+11$ \\
\hline $\mathrm{THC}$ & $3517.29(910.69,6123.89)$ & Infinity (1.78E+147, Infinity) & Infinity, 3.57E+147 \\
\hline THC: CBD & $946.55(214.43,1678.68)$ & $3.75 E+142(1.34 E+35,1.05 E+250)$ & $7.51 E+142,2.69 E+35$ \\
\hline \multicolumn{4}{|l|}{ PANEL MODELS } \\
\hline \multicolumn{4}{|c|}{ Interactive Model - Including Sociodemographics } \\
\hline $\mathrm{THC}$ & $7726.08(3068.06,12,384.1)$ & Infinity $(9.29 E+186$, Infinity) & Infinity, Infinity \\
\hline THC: CBD & $2899.61(1040.91,4758.31)$ & $1.47 \mathrm{E}+176(2.93 \mathrm{E}+63,7.39 \mathrm{E}+288)$ & Infinity, 5.86E+63 \\
\hline THC: CBG: CBD & $202.14(44.72,359.56)$ & $1.91 \mathrm{E}+12(545.0179,6.69 \mathrm{E}+21)$ & $3.82 \mathrm{E}+12,1.09 \mathrm{E}+03$ \\
\hline \multicolumn{4}{|c|}{ Sociodemographic Interactive Model - 1 Lag } \\
\hline Cigarettes: THC: CBD & $163.56(80.31,246.82)$ & $4.75 \mathrm{E}+08(1.85 \mathrm{E}+04,1.21 \mathrm{E}+13)$ & $9.50 \mathrm{E}+08,3.71 \mathrm{E}+04$ \\
\hline Cigarettes: THC & $719.66(347.71,1091.61)$ & $1.50 \mathrm{E}+38(3.05 \mathrm{E}+18,7.39 \mathrm{E}+57)$ & $3.01 \mathrm{E}+38,6.11 \mathrm{E}+18$ \\
\hline \multicolumn{4}{|l|}{ Additive Model - Drugs } \\
\hline Cannabis & $10.61(4.7,16.52)$ & $5.66 \mathrm{E}+19(8.37 \mathrm{E}+08,3.82 \mathrm{E}+30)$ & $1.13 E+20,1.67 E+09$ \\
\hline \multicolumn{4}{|l|}{ Interactive Model - Drugs } \\
\hline Cannabis & $10.61(4.7,16.52)$ & $44.75(5.40,370.45)$ & $89.06,10.29$ \\
\hline \multicolumn{4}{|l|}{ Interactive Model - Drugs, 2 Lags } \\
\hline Cannabis & $241.68(65.24,418.12)$ & $3.25 E+08(204.43,5.18 E+14)$ & $6.51 E+08,408.35$ \\
\hline \multicolumn{4}{|l|}{ THC } \\
\hline $\mathrm{THC}$ & $8.14(4.27,12)$ & $19.67(4.78,80.93)$ & $38.84,9.03$ \\
\hline \multicolumn{4}{|l|}{ Cannabigerol } \\
\hline Cannabigerol & $7.54(3.14,11.94)$ & $14.30(3.04,67.26)$ & $28.10,5.53$ \\
\hline \multicolumn{4}{|l|}{ Additive Model - Drugs \& Cannabinoids } \\
\hline $\mathrm{THC}$ & $8.14(4.27,12)$ & $18.91(4.68,76.34)$ & $37.31,8.84$ \\
\hline \multicolumn{4}{|c|}{ Interactive Model - Drugs \& Cannabinoids } \\
\hline THC & $8.14(4.27,12)$ & $19.67(47.78,80.94)$ & $38.84,9.04$ \\
\hline \multicolumn{4}{|c|}{1 Spatial Lag-Interactive Model, $T H C$ * CBD } \\
\hline Cigarettes: CBD & $792.04(292.87,1291.2)$ & $2.15 E+125(3.19 E+46,1.45 E+204)$ & $4.31 E+125,6.39 E+46$ \\
\hline Cigarettes: THC: CBD & $912.27(282.87,1541.68)$ & $2.29 E+144(9.107 E+44,5.77 E+243)$ & $4.58 \mathrm{E}+144,1.83 \mathrm{E}+45$ \\
\hline Cigarettes:THC & $3167.74(941.61,5393.88)$ & Infinity (5.16E+149, Infinity) & Infinity, 1.03E+150 \\
\hline \multicolumn{4}{|c|}{1 Spatial Lag - Interactive Model, THC * CBG } \\
\hline Cigarettes: THC: CBG & $855.74(286.28,1425.2)$ & $8.09 E+135(4.45 E+45,1.47 E+226)$ & $1.61 \mathrm{E}+136,8.91 \mathrm{E}+45$ \\
\hline
\end{tabular}


Table 23 (continued)

\begin{tabular}{|c|c|c|c|}
\hline Parameter & Estimate (C.I.) & R.R. (C.I.) & E-Values \\
\hline Cigarettes: THC & $2980.74(841.4,5120.08)$ & Infinity (2.06E+134, Infinity) & Infinity, 4.14E+134 \\
\hline Cigarettes: CBG & $755.71(171.07,1340.34)$ & $1.05 E+120(2.27 E+27,4.83 E+212)$ & $2.09 E+120,4.54 E+27$ \\
\hline \multicolumn{4}{|c|}{1 Spatial, 1 Temporal Lag Cannabinoids } \\
\hline Cigarettes: THC: CBD & $1394.48(386.59,2402.38)$ & 4.17E+192 (4.79E+53, Infinity) & Infinity, 9.59E+53 \\
\hline Cigarettes: THC: THC.Spatial: CBD & $1384.11(374.49,2393.72)$ & $1.53 \mathrm{E}+1981(1.02 \mathrm{E}+52$, Infinity) & Infinity, 2.05E+52 \\
\hline Cigarettes: THC & $5000.58(1323.37,8677.78)$ & Infinity (6.65E+183, Infinity) & Infinity, Infinity \\
\hline Cigarettes: THC: THC.Spatial & $4975.93(1182.67,8769.19)$ & Infinity (2.62E+164, Infinity) & Infinity, Infinity \\
\hline Cigarettes: CBD & $522.98(44.27,1001.7)$ & $1.74 \mathrm{E}+72(1.77 \mathrm{E}+06,1.70 \mathrm{E}+138)$ & $3.47 \mathrm{E}+72,3.54 \mathrm{E}+06$ \\
\hline \multicolumn{4}{|c|}{1 Spatial, 2 Temporal Lags Cannabinoids } \\
\hline Cigarettes: CBD & $48,350.5(20,095.92,76,605.08)$ & Infinity (Infinity, Infinity) & Infinity, Infinity \\
\hline Cigarettes: THC & $217,699.3(89,605.46,345,793.14)$ & Infinity (Infinity, Infinity) & Infinity, Infinity \\
\hline Cigarettes: THC: CBD & $76,973.5(31,232.59,122,714.41)$ & Infinity (Infinity, Infinity) & Infinity, Infinity \\
\hline THC & $11,707.8(4631.81,18,783.79)$ & Infinity (1.01E+190, Infinity) & Infinity, Infinity \\
\hline THC:THC.Spatial & $19,063(7395.32,30,730.68)$ & Infinity $(2.51 \mathrm{E}+303$, Infinity $)$ & Infinity, Infinity \\
\hline \multicolumn{4}{|c|}{ Interactive Model - Including Sociodemographics } \\
\hline THC & $37.58(9.36,65.79)$ & $1.86 \mathrm{E}+06(37.31,9.29 \mathrm{E}+10)$ & $3.72 \mathrm{E}+06,74.13$ \\
\hline THC: CBG & $6.95(0.33,13.56)$ & $14.44(1.14,1852.37)$ & $28.36,1.54$ \\
\hline \multicolumn{4}{|l|}{ Sociodemographic Interactive, 1 Lag } \\
\hline Cigarettes:THC & $46.25(16.84,75.67)$ & $3.85 E+05(109.80,1.35 E+09)$ & $7.71 E+05,219.10$ \\
\hline \multicolumn{4}{|l|}{ Sociodemographic Interactive, 2 Lags } \\
\hline Cigarettes: THC: CBD & $6151.83(2693.75,9609.91)$ & $1.63 E+301(1.72 E+132$, Infinity) & Infinity, 3.45E+132 \\
\hline Cigarettes:THC & $22,951.53(9883.29,36,019.77)$ & Infinity (Infinity, Infinity) & Infinity, Infinity \\
\hline Cigarettes: CBD & $4078.6(1248.29,6908.9)$ & $5.02 \mathrm{E}+199(2.51 \mathrm{E}+61$, Infinity $)$ & Infinity, 5.02E+61 \\
\hline
\end{tabular}

Eyes

- Splits the single eye field into two halves, right and left [92]

- Induces the outgrowth of the optic cup from the forebrain which becomes the optic nerve and then the optic vesicle and later neural retina

- The bulging frontal lobe of the forebrain secretes shh to induce an ectodermal organizing centre in the overlying skin called the frontonasal ectodermal zone which controls the development of the cheeks and nose again by the secretion of shh

- Induction of the ventral and nasal retinae of the eye

- Acts as a repulsive signal guiding axonal growth of retinal ganglion cells

- Retinal patterning [92]

Ears

- Ear specification - shh specifies ventrality in the developing otocyst [92]
Mouth

- Controls mouth formation and size of mouth [92]

- Breaks down the oropharyngeal membrane

Respiratory

- Tips of outgrowing lung buds [92]

Gastrointestinal Tract

- Upper and lower Intestinal portals [92]

- Controls specification of the foregut

- Shh secreted from the esophageal mucosa control radial specification of the esophagus and inhibits muscle development in the submucosa,

- Shh signalling from the gastric mucosa controls smooth muscle development

- Gastric development and enlargement [92]

- Shh secreted from the intestinal mucosa control radial specification of the intestinal and inhibits muscle development in the submucosa, 
Table 24 Obstructive Genitourinary Defects - E-Values from Space-Time Regression Models

\begin{tabular}{|c|c|c|c|}
\hline Parameter & Estimate (C.I.) & R.R. (C.I.) & E-Values \\
\hline \multicolumn{4}{|l|}{ Additive Model - Drugs } \\
\hline Cannabis & $10.61(4.7,16.52)$ & $5.66 \mathrm{E}+19(8.37 \mathrm{E}+08,3.82 \mathrm{E}+30)$ & $1.13 \mathrm{E}+20,1.67 \mathrm{E}+09$ \\
\hline \multicolumn{4}{|l|}{ Interactive Model - Drugs } \\
\hline Cannabis & $10.61(4.7,16.52)$ & $44.75(5.40,370.45)$ & $89.06,10.29$ \\
\hline \multicolumn{4}{|l|}{ Interactive Model - Drugs, 2 Lags } \\
\hline Cannabis & $241.68(65.24,418.12)$ & $3.25 \mathrm{E}+08(204.43,5.18 \mathrm{E}+14)$ & $6.51 \mathrm{E}+08,408.35$ \\
\hline \multicolumn{4}{|l|}{ THC } \\
\hline THC & $8.14(4.27,12)$ & $19.67(4.78,80.93)$ & $38.84,9.03$ \\
\hline \multicolumn{4}{|l|}{ Cannabigerol } \\
\hline Cannabigerol & $7.54(3.14,11.94)$ & $14.30(3.04,67.26)$ & $28.10,5.53$ \\
\hline \multicolumn{4}{|c|}{ Additive Model - Drugs \& Cannabinoids } \\
\hline $\mathrm{THC}$ & $8.14(4.27,12)$ & $18.91(4.68,76.34)$ & $37.31,8.84$ \\
\hline \multicolumn{4}{|c|}{ Interactive Model - Drugs \& Cannabinoids } \\
\hline $\mathrm{THC}$ & $8.14(4.27,12)$ & $19.67(47.78,80.94)$ & $38.84,9.04$ \\
\hline \multicolumn{4}{|c|}{1 Spatial Lag - Interactive Model, THC *CBD } \\
\hline Cigarettes: CBD & $792.04(292.87,1291.2)$ & $2.15 \mathrm{E}+125(3.19 \mathrm{E}+46,1.45 \mathrm{E}+204)$ & $4.31 \mathrm{E}+125,6.39 \mathrm{E}+46$ \\
\hline Cigarettes: THC: CBD & $912.27(282.87,1541.68)$ & $2.29 E+144(9.107 E+44,5.77 E+243)$ & $4.58 \mathrm{E}+144,1.83 \mathrm{E}+45$ \\
\hline Cigarettes:THC & $3167.74(941.61,5393.88)$ & Infinity (5.16E+149, Infinity) & Infinity, $1.03 E+150$ \\
\hline \multicolumn{4}{|c|}{1 Spatial Lag - Interactive Model, THC * CBG } \\
\hline Cigarettes: THC: CBG & $855.74(286.28,1425.2)$ & $8.09 E+135(4.45 E+45,1.47 E+226)$ & $1.61 \mathrm{E}+136,8.91 \mathrm{E}+45$ \\
\hline Cigarettes: THC & $2980.74(841.4,5120.08)$ & Infinity $(2.06 \mathrm{E}+134$, Infinity) & Infinity, 4.14E+134 \\
\hline Cigarettes: CBG & $755.71(171.07,1340.34)$ & $1.05 \mathrm{E}+120(2.27 \mathrm{E}+27,4.83 \mathrm{E}+212)$ & $2.09 \mathrm{E}+120,4.54 \mathrm{E}+27$ \\
\hline \multicolumn{4}{|c|}{1 Spatial, 1 Temporal Lag Cannabinoids } \\
\hline Cigarettes: THC: CBD & $1394.48(386.59,2402.38)$ & 4.17E+192 (4.79E+53, Infinity) & Infinity, 9.59E +53 \\
\hline Cigarettes: THC: THC.Spatial: CBD & $1384.11(374.49,2393.72)$ & $1.53 \mathrm{E}+1981(1.02 \mathrm{E}+52$, Infinity $)$ & Infinity, $2.05 E+52$ \\
\hline Cigarettes: THC & $5000.58(1323.37,8677.78)$ & Infinity $(6.65 E+183$, Infinity $)$ & Infinity, Infinity \\
\hline Cigarettes: THC:THC.Spatial & $4975.93(1182.67,8769.19)$ & Infinity $(2.62 E+164$, Infinity) & Infinity, Infinity \\
\hline Cigarettes: CBD & $522.98(44.27,1001.7)$ & $1.74 \mathrm{E}+72(1.77 \mathrm{E}+06,1.70 \mathrm{E}+138)$ & $3.47 \mathrm{E}+72,3.54 \mathrm{E}+06$ \\
\hline \multicolumn{4}{|c|}{1 Spatial, 2 Temporal Lags Cannabinoids } \\
\hline Cigarettes: CBD & $48,350.5(20,095.92,76,605.08)$ & Infinity (Infinity, Infinity) & Infinity, Infinity \\
\hline Cigarettes: THC & $217,699.3(89,605.46,345,793.14)$ & Infinity (Infinity, Infinity) & Infinity, Infinity \\
\hline Cigarettes: THC: CBD & $76,973.5(31,232.59,122,714.41)$ & Infinity (Infinity, Infinity) & Infinity, Infinity \\
\hline THC & $11,707.8(4631.81,18,783.79)$ & Infinity (1.01E+190, Infinity) & Infinity, Infinity \\
\hline THC:THC.Spatial & $19,063(7395.32,30,730.68)$ & Infinity (2.51E+303, Infinity) & Infinity, Infinity \\
\hline \multicolumn{4}{|c|}{ Interactive Model - Including Sociodemographics } \\
\hline $\mathrm{THC}$ & $37.58(9.36,65.79)$ & $1.86 \mathrm{E}+06(37.31,9.29 \mathrm{E}+10)$ & $3.72 \mathrm{E}+06,74.13$ \\
\hline THC: CBG & $6.95(0.33,13.56)$ & $14.44(1.14,1852.37)$ & $28.36,1.54$ \\
\hline \multicolumn{4}{|l|}{ Sociodemographic Interactive, 1 Lag } \\
\hline Cigarettes:THC & $46.25(16.84,75.67)$ & $3.85 \mathrm{E}+05(109.80,1.35 \mathrm{E}+09)$ & $7.71 E+05,219.10$ \\
\hline \multicolumn{4}{|l|}{ Sociodemographic Interactive, 2 Lags } \\
\hline Cigarettes: THC: CBD & $6151.83(2693.75,9609.91)$ & $1.63 \mathrm{E}+301(1.72 \mathrm{E}+132$, Infinity $)$ & Infinity, 3.45E+132 \\
\hline Cigarettes: THC & $22,951.53(9883.29,36,019.77)$ & Infinity (Infinity, Infinity) & Infinity, Infinity \\
\hline Cigarettes: CBD & $4078.6(1248.29,6908.9)$ & $5.02 \mathrm{E}+199(2.51 \mathrm{E}+61$, Infinity $)$ & Infinity, $5.02 E+61$ \\
\hline
\end{tabular}

- The muscularis mucosae of the small intestine develops much later in foetogenesis when the shh gradients have declined

- Intestinal elongation
- Controls the activity of the gut stem cells deep in the intestinal crypts

- Rostral and caudal intestinal portals

- Controls the development of the anal opening

- Controls pancreas development [92] 


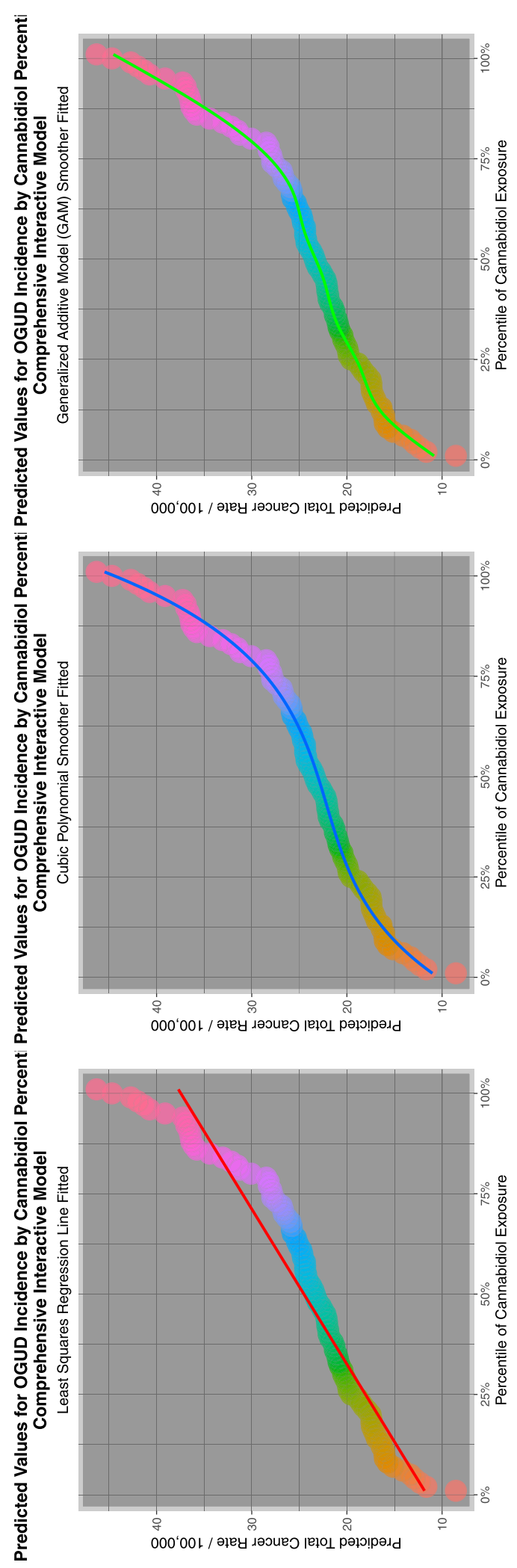

Fig. 19 Modelled rates of obstructive genitourinary defect with rising cannabidiol exposure in a geospatial model lagged to two years

Cardiac

- Maintains cardiogenic proliferation in the secondary heart field [93]

- The shh-dependent secondary heart field contributes to the conoventricular outflow tract [94]

- Shh controls elongation of the conoventricular outflow tract via shh-dependent progenitors [94]

- Shh is essential for aortic arch development [95]

- Shh control outflow tract development $[96,97]$

- Shh is critical in cardiovascular development [98]

- Shh plays a critical role in neural crest cell specification some of which contribute to cardiac cells [99]

\section{Vascular}

- Induces formation of the dorsal aortae [100]

- Controls formation and remodelling of branchial arch blood vessels [101]

- Together with BMP and notch signalling shh is critically involved with induction of the first dedicated haemopoietic cells which arise in the fusing dorsal aortae

- Arterial differentiation is induced in a molecular cascade which commences with shh signaling to VEGFA and notch from a general endothelial background of angioblasts $[92,102,103]$

\section{Genitourinary}

- Contributes to bladder growth and sufficiency [92] - Contributes as a trophic factor to development and outgrowth of the genital tubercle under the influence of shh derived from the urethral endoderm [92]

Limbs

- Zone of polarizing activity in limb formation [92]

- Key organizer of the patterning of the digits [92]

- Hair buds development 
Therefore the recent demonstration therefore that cannabidiol and THC inhibit shh signalling necessarily carries major implications for cannabinoid-related teratogenesis [42]. These cannabinoids were noted to both depress shh and Gli1 mRNA and induce the formation of a CB1R-smoothened ("smoothened" is the effector molecule of the shh "patched" receptor) heteromer which reverses the polarity of downstream signalling of smoothened. These authors noted that the critical period for foetal development in this regard is the third to fourth week of gestation in the embryonal period of development when many women are unaware that they are pregnant.

Interference with shh-dependent processes at key stages of development will likely result in the following anomalies which have been described in various studies as being cannabis-related:

- Exencephaly [11, 104]

- Encephalocele $[13,17]$

- Deficiencies in spinal column formation - myelocele and meningomyelocele [13],

- Mental deficiencies such as ADHD and autism spectrum from deficient forebrain differentiation $[10,66$, $88,105]$

- Lowered tone and motor control as has been described in children experiencing prenatal cannabinoid exposure [10, 106-109]

- Impaired visuomotor and executive processing seen in PCE children [110-112]

- Cleft lip and palate (USA- present study)

- Holoprosencephaly [42] including cyclopia (single eye) (USA- present study)

- Respiratory $[18,20]$

- Limb defects [11-13, 18, 20, 104, 113] (USA- present study)

- Vascular catastrophes - in limbs [13] (USA- present study), body wall closure [7, 8, 13, 114-118]

- Epispadias, hypospadias [20] (USA- present study)

- Obstructive Genitourinary defect (USA- present study)

- Gastrointestinal stenoses and atresias (USA- present study)

- Anorectal agenesis

It has been reported by many investigators that cannabinoids reduce cell growth and reduce synthesis of the macromolecules of life such as DNA, RNA and proteins including histones $[12,23,24,26-32$, 119-122].

The inhibition of cell growth and division would explain many features of cannabis teratogenesis including: i) Failure of the anterior and posterior neuropores to close, resulting in encephalocele, exencephaly and spina bifida respectively;

ii) Cleft lip and palate due to failure of the facial and palatal processes to properly fuse

iii) Several cardiovascular defects including:

a. Atrial septal defect secundum, where the atrial septal folds fail to grow across the defect

b. Ventricular septal defects where the various components of the ventricular wall fail to join across the defect

c. Stenoses and atresias of the heart valves

d. Defective development of the great vessels, which have a very complex developmental course

iv) Body wall defects

v) Limb defects, where failure or interruption of cell division at key period of limb bud outgrowth interrupts the normal sequence of events required for normal limb development affecting:

a. The whole limb

b. The upper or lower segments of the limb

c. Digital development of fingers and toes

vi) Gastrointestinal stenoses and atresias including:

a. Esophageal atresia [7] (USA- present study)

b. Small intestinal stenosis and atresia (USA- present study)

c. Large intestinal stenosis and atresia (USA- present study)

d. Biliary stenosis and atresia (USA- present study)

e. Anorectal stenosis and atresias (USA- present study)

vii) Arterial vascular catastrophes

a. Limb development

b. Body wall - omphalocele, gastroschisis, diaphragmatic hernia

As shown above shh is known to be a key morphogen directing the differentiation of the arterial tree and its inhibition can be expected to disrupt normal vasculogenic and arterial supply of key tissues. Cannabinoids are also vasoactive [123]. Both type 1 and 2 cannabinoid receptors (CB1Rs and CB2Rs) along with other receptor subtypes have been described on the vasculature [123]. Cannabinoids acting at CB1Rs are often proinflammatory and vasoconstrictive [123-127]. Such 
vascular defects could be involved with the genesis of various congenital anomalies including:

i) Body wall defects (gastroschisis and omphalocele) cocaine and various vasoconstrictive antihistaminic drugs are known to be associated with gastroschisis [128-133] and cannabinoids may act similarly at least in the foetal period of development

ii) Gastrointestinal stenoses and atresias

iii) Limb development as the developing limb anlage is highly vascular dependent any interruption of its blood supply will necessarily truncate development.

Hence it could be said that the full spectrum of cannabinoid-induced embryopathy follows to a close approximation a picture of shh mutation or deficit. The point has previously been made that embryonic shh deficiency causes a wide variety of congenital defects including effects on vertebra, anal atresia, cardiovascular anomalies, tracheoesophageal fistula, renal defects and limb defects (VACTERL syndrome) [134]. These defects also have similarities both to fetal alcohol syndrome [42] and Di George I Velocardiofacial (palatocardiofacial) syndrome which may also include kidney and intellectual problems [135].

\section{Other genotoxic mechanisms}

In addition to direct and indirect interactions with specific morphogen pathways cannabinoids have also been shown to interact deleteriously with chromosomes, DNA, the epigenome and mitochondrial-metabolic-epigenomic pathways. These are reviewed in a companion manuscript and have been considered elsewhere [18-20, $24,28,31,37,38,41,91,113,136-142]$.

\section{Specific organ systems \\ Heart}

In Hawaii five cardiovascular defects were related to elevated cannabis use, atrial and ventricular septal defects, pulmonary valve atresia and stenosis, tetralogy of Fallot and hypoplastic left heart syndrome [13]. In Colorado four cardiovascular defects rose across time with increasing community cannabinoid penetration, namely atrial septal defect, ventricular septal defect, patent ductus arteriosus and anomalies of the pulmonary artery [20]. In Canada total cardiovascular defects were related to increased cannabis use [18]. In Australia total cardiovascular defects, atrial and ventricular septal defects, transposition of the great arteries, tetralogy of Fallot and patent ductus arteriosus occurred with higher incidence in high cannabis using areas [19]. They also featured prominently in the present US overview.
It is important to appreciate that heart development occurs by including cells from many loci in the embryo including the primary and secondary heart fields, proepicardium, Juxtacardiac field [143], cardiac neural crest and neural crest [92].

Major morphogens acting are retinoic acid, FGFs and sh. Neuregulin is involved in the induction of both the heart valves and also the subendocardial electrical conducting system of the heart [92].

It therefore follows that heart and great vessels form as a result of a carefully orchestrated sequential complementation of progenitor cells from many areas, some quit remote from the cardiogenic field itself [92]. It is also apparent that numerous genes and transcription factors are involved in this process [92].

Given the wide diversity of cannabinoid actions in a wide variety of cell types it seems particularly unlikely that cannabinoids would not impact this delicate and intricate process at many points.

The numerous interactions of shh with both heart and great vessel formation were enumerated above.

\section{Respiratory defects}

Respiratory defects were noted to be elevated in the high cannabis using areas of Colorado and Canada [18, 20]. Shh is noted to be centrally involved in the budding and development of the respiratory tree [92].

\section{Face}

In the Hawaiian series incidence rates of cleft lip and palate together with anotia / microtia were elevated by prenatal cannabis exposure [13]. Microphthalmia was non-significantly elevated. In Canada facial clefts were non-significantly elevated [18]. In Australia facial and ear anomalies were non-significantly elevated [19].

As was noted above shh plays a large role in face development through the frontal facial organizer, at the tip of the frontonasal processes which form the sides of the cleft lip, at the tips of the palatal shelves, in the tongue, teeth, taste buds and filiform papillae [92].

Alcohol and steroidal alkaloids are known to disrupt shh signalling in the face [144].

\section{Gastrointestinal tract}

The Hawaiian series noted that several gastrointestinal anomalies were elevated following prenatal cannabis exposure including esophageal atresia, pyloric stenosis, and large bowel stenoses and atresias including anorectal atresia [13]. In Australia small intestinal stenosis was 
identified positively [19]. Gastrointestinal anomalies featured prominently in the present analysis including particularly small intestinal stenosis and atresia which was linked with cannabidiol use both causally and in a spacetime context.

The prominent involvement of shh and major morphogens in the growth and development of all parts of the gastrointestinal tract was described above [92].

\section{Urinary tract}

Given the above notes on the location of shh in the genitourinary system it is of interest that obstructive genitourinary defects were identified both in Hawaii and in the present US survey series [13]. Hypospadias was identified positively in Australia [19].

\section{Body wall anomalies}

Gastroschisis and diaphragmatic hernia have previously been noted to be linked with prenatal cannabis exposure by CDC and NBDPN researchers [7] although gastroschisis was not positively identified in the present investigation [7]. In Colorado gastroschisis and diaphragmatic hernia were positively identified [20].

\section{Limbs}

Limb reductions were noted as significant correlates in the continuous bivariate analysis of THC and cannabis with minimal E-Values of 1.89 and 9.53. Leg reductions were noted as significant correlates of cannabidiol, THC and cannabis with minimal E-Values of 2.38, 1.32, and 2.57 (Tables $6,7,8$ ). They were not seen in association with tobacco, alcohol or cocaine exposure. This finding is consistent with the arm reduction anomalies reported from Hawaii following prenatal cannabis exposure [13], the elevation of total congenital anomalies seen in Canada which also may have included limb reductions [18] and preclinical studies [11, 12, 104]. Cannabis of course is well known to interfere with both cellular division including macromolecular synthesis and blood vessel sprouting. Blood vessels are known to have high density cannabinoid receptors which are known to be frequently pro-inflammatory and vasoactive [123-127]. Moreover limb outgrowth occurs in a tight time window during embryogenesis [145]. It is therefore possible that cannabinoid exposure during this critical window of development interferes with cellar division in the limb bud and vascular budding and outgrowth thereby compromising limb development.

It is of interest that arm reduction anomalies along with polydactyly and syndactyly were noted to have occurred with increased incidence rates following prenatal cannabis exposure in the Hawaiian series, and leg anomalies rates rose in the present US series [13]. Polydactyly and syndactyly and total musculoskeletal anomalies rose in Colorado with cannabis legalization [20]. It is difficult to comment on the major limb anomalies as it is a congenital anomaly for which ETOPFA may be practised at high rates. In the Australian series there was a non-significant trend to higher rates of major arm and leg anomalies in the high cannabis using areas [19]. Similarly outbreaks of major limb anomalies were noted in both France and Germany [45, 47, 48, 50] in recent years where cannabinoids have been allowed to enter the food chain, but not in nearby Switzerland where this is not permitted.

Major morphogens involved in early limb development are opposing gradients of the Fibroblast Growth Factors (FGF) and Wnt on the one hand and retinoic acid on the other. Limb length is controlled by Hox genes D-9 to D-13. Specification and formation of the fingers and toes is controlled by alternating interactions and gradients between sonic hedgehog, gremlin and FGF4 and by manipulating these gradients and gene dosages experimentally one is able to control various malformations in a predictable manner [145].

It is of interest therefore that there are at least three major pathways by which cannabinoids can interfere with limb bud development and outgrowth:

i) Direct inhibition of cell division and cell growth

ii) Direct and indirect blockades of shh gradients from the zone of polarizing activity in the inferior axillary region and along the posterior edge of the limb and in the digital rays

iii) Vasculopathic mechanisms whereby interference with the ingrowing blood supply compromises limb development.

It is important to note that limb development is strictly sequential so that a block at critical developmental time periods will inevitably block subsequent steps. It is easy to appreciate in such a paradigm that significant cannabinoid intake in such critical windows of gestation may have potentially catastrophic implications for limb growth and development.

It is also noteworthy that cannabis shares many of the mechanisms of action of thalidomide [146-152] an agent which is notorious for interfering with limb outgrowth and bony skeletal development, albeit at higher potency [53, 146, 151, 153-156].

\section{Chromosomal defects}

Downs syndrome was identified positively in Hawaii, Colorado, Australia and Canada as well as in the present 
analysis of both categorical and continuous ETOPFAcorrected data [13, 18-20]. Chromosomal defects were found to be elevated in Canada and Australia $[18,19]$ as well as in the present US survey.

Several mechanisms of indirect chromosomal clastogenicity and DNA breakage have been described [24, 26, $28,33,138]$.

\section{Interactions of cannabinoids with other major morphogen systems}

Interaction between FGF (Fibroblast Growth Factor) and endocannabinoid systems have also been described [157, 158] including transactivation of the FGF1R by CB1R [159].

Interactions between cannabinoids and bone morphogenetic proteins have also been described [160-162].

Interactions between cannabinoids and retinoic acid signalling have been described [163-165].

Interactions between cannabinoids and notch signalling have also been reported [166-172].

Interactions between cannabinoids and Wnt signalling have also been reported [173-179].

Interactions between cannabinoids and hippo have been reported [140].

Cannabinoids also interact with the neurexin-neuroligin system [180-182] which is key to the architecture and development of neural synapses.

Cannabinoids also interact with the slit-robo system $[168,169,183]$ which control arterial pathfinding and also axonal growth cone steering mechanisms [92, 171, $184,185]$. Slit-robo signalling is also one of the major morphogens directing and controlling the exuberant outgrowth of the massive human neocortex $[183,186]$.

\section{Commonality}

Given this plethora of actions of actions between cannabinoids and the major morphogens of human and mammalian development one might well wonder why such anomalies are not becoming much more common. There are several parts to this answer. One factor is that the birth defect data from states where cannabis is legal such as Washington state and Oregon are almost non-existent. Data from Colorado shows a dramatic rise in congenital anomalies across the period of legalization as has been mentioned elsewhere [20]. Also since cannabinoids are involved in virtually every aspect of reproduction including gamete formation and meiotic divisions, the function of supporting granulosa and Sertoli cells in ovary and testis, cells placentation, implantation, sperm fertility and hyperactivation, ovarian signals to the sperm and cell division at the early zygote, morula and embryonic stages a high rate of foetal loss is expected from severe anomalies which does not necessarily appear on lists of birth defects, but is chronicled in case series such as that described above from Washington D.C [14, 15]. Moreover the actual state level ETOPFA rate likely varies from place to place and this is a major determinant of the rates of many serious CAs.

\section{Causal assignment}

Two of the commonest criticisms made of observational studies are that the exposure of interest is not distributed randomly across all experimental subjects, and that there may be some uncontrolled confounding operating from some unmeasured variables which account for the observed effect and for which the observed variables are acting merely as surrogates or substitute markers.

The first criticism is answered in the present study by the use of inverse probability weighting of the exposed groups. It is well established that the use of this procedure across observations transforms a merely observational dataset into a pseudo-randomized one from which causal conclusions can properly be drawn by comparing exposure groups. This technique is particularly suitable for those comparisons which would not generally be ethical to apply in randomized controlled studies, such as antenatal exposures.

The second criticism is addressed herein by the use of E-Values. E-Values, or expected values, calculate the degree of correlation required of some unknown confounding variable with both the exposure and the outcome to explain away the observed effect. The literature mentions that values above 1.25 are generally considered to indicate causal effects [67]. The E-value for the lung cancer - tobacco relationship is 9 which is considered high $[67,68,86]$. It is clear from the present study that many of the E-Values quoted are much higher than this gold standard metric.

Moreover it is entirely proper to use E-Values freely in relation both to specific models (which have model standard deviations) and to final predictive models as has been done in the present report [69].

One also notes that for two congenital anomalies we have conducted multiple regression by several techniques which have very similar conclusions. Moreover for these defects we have shown in their intrinsic natural spacetime context that these relationships are conserved and indeed amplified.

Furthermore our results are also consistent with a long, robust and highly consistent tradition of laboratory and preclinical evidence as noted above.

As judged by the criteria of causation proposed by Hill [187] the present results fulfil the criteria of strength 
of association, consistency across studies in the manner described, specificity amongst substance exposures, temporality of sequence, coherence with known data, biological plausibility as described in the above mechanistic discussion, biological dose-response curve, analogy with similar situations in other places and experimental confirmation.

\section{Generalizability}

The present study has several advantages. Its study subject is a sizeable base population comprising a national census birth population in excess of 18 million births, from a notional year-on-year aggregated annualized total population of over 2 billion persons. Drug use data is taken from a well verified nationally representative survey which has been faithfully repeated annually for several decades now with very little important change which greatly facilitates comparison between periods. Advanced statistical methods are employed on both the aggregate dataset of all defects and two congenital anomalies in particular. The techniques both of formal spacetime analysis and of causal inference have been utilized. For these reasons internal to the study we are confident that the present work is widely applicable across the globe. Results reported herein strongly indicate that in those third world nations where cannabis is known to be much more widely used the results are expected to be much more severe than those reported for this nation where historically cannabis use was relatively restricted until recent years.

The demonstration that many of these effects give the appearance on bivariate analysis of being truly causal also necessarily implies that the results are truly biological and widely generalizable.

The present work is also entirely consistent with a large and growing external body of evidence from particular states within USA, namely Colorado and Hawaii $[13,20]$ and also from Australia and Canada which attest to the concordance with the findings reported herein [17-19].

Another important body of work which supports the present results is the preclinical literature which the present results closely replicate. As was noted above in fact virtually all of the mentioned congenital anomalies have been positively identified in the present study.

Hence for this variety of both internal and external reasons we feel that the findings in the present study are widely generalizable with the primary caveat that in nations where cannabis is more widely available we believe that the findings would be of even greater concern in those cases where reliable datasets exist for its accurate assessment.

\section{Strengths and limitations}

In considering the strengths and limitations of the present study it is important to clarify exactly what this study is and what it is not. The present study sets out to present a broad overview of the apparent relationship of the US teratological experience to substance exposure in the population during the notional period 2005-2013 when both major datasets are available. It goes on to explore two particular anomalies in detail from both a causal inference and geotemporospatial perspective as examples of the manner in which such analyses can be carried forward using more versatile analytical techniques on extent data series. For these reasons we feel it is premature to propose a list of cannabinoid related congenital anomalies and limit ourselves merely to noting that the issue is of considerable concern and well warrants further advanced statistical, epidemiological and basic science investigation. Thus our study is not the last word on US substance-related teratology, but in that it applies a series of advanced sequential linear and predictive modelling and sophisticated analytical space-time and causal inferential techniques our study is more like the first word opening an important discussion which has not been well addressed in recent years.

This study has several strengths including using a nationwide census database for congenital anomalies, using a large well validated nationally representative sample of the non-institutionalized US population, using the major techniques of quantitative causal inference namely inverse probability weighting and E-values, and geospatial regression across space and time simultaneously to assess these roles, and continues by studying the predicted values from space-time models to examine the way in which increasing cannabidiol exposure can be related spatiotemporally to increasing dose-effect relationships. The analytical techniques featuring linear models in tidy format conducted serially on 62 congenital anomalies in purrr allow direct comparison of models within the same statistical run. The use of multi-facetted plots allow the direct visual comparison of the effect on multiple congenital anomalies to be visually inspected at a glance, and similarly between plot comparisons allows the effects of various environmental teratogens to be directly compared. Graphical presentations of E-Values also allow the quantitative and causal significance of findings between substances to be directly compared.

The limitations of this study relate to the limitations of its design. In common with most epidemiological studies individual patient level exposure data was not available to it. Obvious ways in which the present work might be extended such as by increasing the geospatial resolution 
of the work and by increasing the numbers of congenital anomalies for which detailed regression results are presented are outside the ambit of the present study, and represent a fertile area for future workers. NBDPN may be able to further extend the dataset by completing missing data fields. Moreover perhaps the most definitive technique by which to study these data would include the use of inverse probability weighting in spatiotemporal models. It may become possible with time to employ a weighting term which is actually a product of two lists of weights, one being a sparse geospatial matrix and one being IPW, similar to a current implementation in the $\mathrm{R}$ "survey" package. Since such techniques have not been developed at the time of writing it has not been possible to deploy them on these topics. In their stead multiple IPW causal models have been used to address pseudorandomization and complete these gaps. This also represents an important area for future statistical methodological development. As the USA moves increasingly towards population wide exposure to cannabinoids the importance of quantifiable continuous measures of exposure to various cannabinoids, for example by epigenomic and or glycomic criteria proportionately increases as has previously been noted [91]. State level anomaly-specific ETOPFA rates were not available to this work and ETOPFA rates had to be estimated from the published literature. Their addition to the present dataset would improve the quality and accuracy of the various estimates used.

\section{Conclusion}

In summary we note that bivariate analysis of ETOPFAcorrected $\mathrm{CA}$ incidence against state-based substance exposure rates indicates that cannabis and estimated THC are more important environmental teratogens than tobacco, and cannabidiol is likely more important in these metrics than either binge or regularly consumed alcohol. Elevated E-values for many defects indicates that a causal relationship is likely. Small intestinal stenosis and atresia and obstructive genitourinary defects were studied in detail by inverse probability weighted mixed effects, robust and panel regression and by space-time regression and by predictive modelling in spatiotemporal models where these findings were all strongly confirmed and again were shown to be epidemiologically causal in nature. Results are consistent and concordant with several decades of preclinical and laboratory work implicating cellular pathways at chromosomal, genomic, epigenomic and mitochondriopathic levels and with interruption of major embryonal-foetal morphogen gradients particularly sonic hedgehog and with patterns of fetotoxicity and embryotoxicity observed in preclinical models and fulfil the Hill criteria of causality. The present work is part of an on-going project to further investigate these themes in greater depth and finer detail. Further work by interested groups in related areas is strongly indicated.

The present situation where cannabidiol is widely available across USA and popularly perceived as harmless is unusually uninformed and particularly ill-advised. Our analyses implicate THC, cannabigerol and cannabidiol, and analyses could be presented similarly implicating also cannabinol and cannabichromene. From a public health point of view the present de facto policy of official negligence is at once unjustified and unjustifiable.

Data indicate that cannabinoid teratogenicity including cannabidiol teratogenicity and presumptive genotoxicity are clinically significant and carry far-reaching and multi-generational public health impacts in foetalmaternal and reproductive medicine. We feel that it is important that the transgenerational impacts of general register-wide overviews and surveys such as this be given wide canvas and discussion in the community and assume substantial prominence in the public debate on the proper and proven role of cannabinoids in the global community. Moreover the assignment of proper weight to inheritable considerations is essential to optimally formulate policy which balances the risk-benefit equation relating to the general widespread distribution of known genotoxins such as numerous cannabinoids - including cannabidiol - as indeed genotoxicity and fetotoxicity has always been a foundational cornerstone and was always the conceptual origin of modern drug regulation by national Government agencies.

\footnotetext{
Abbreviations

AFE: Attributable fraction in the exposed; BMP: Bone morphogenetic proteins; CA: Congenital anomaly; CBC: Cannabichromene; CBD: Cannabidiol; CBG: Cannabigerol; CBN: Cannabinol; CDC: Centers for disease control, Atlanta, Georgia; cGAS: Cyclic GMP-AMP Synthase; Dbx: Double homeobox; DEA: Drug enforcement agency; ETOPFA: Early termination of pregnancy for anomaly; ETOPFACAR: Early termination of pregnancy for anomaly -adjusted congenital anomaly rate; E-Value: Expected value; FVV: Fitted values; FGF: Fibroblast growth factor; Fox: Forkhead box; GAM: Generalized additive model; Gli1: Glioma-associated protein 1; IPW: Inverse probability weighting; NBDPN: National birth defects prevention network; Nkx: Homeobox protein Nkx; NSDUH: National survey of drug use and health; OGUD: Obstructive genitourinary defect; OLS: Ordinary least squares; PAR: Population attributable risk; Pax: Paired box; plm: Panel linear model; PR: Prevalence ratio; RDAS: Restricted-use data analysis system; re: Random effects; SAMHDA: Substance use and mental health data archive; SAMHSA: Substance abuse and mental health services administration; sem: Spatial error method; semsrre: Spatial error method, serial autocorrelation and random effects; sf: Simple features (Package in R); SISA: Small intestinal stenosis and atresia; Shh: Sonic hedgehog; splm: Spatial panel linear model; spreml: Spatial panel random effects maximum likelihood; SPDSST: Spatial panel dataset in space-time; sr: Serial correlation; STING: Stimulator of interferon genes; THC: $\triangle 9$-Tetrahydrocannabinol; VEGFA: Vascular endothelial growth factor $A$.
} 


\section{Supplementary Information}

The online version contains supplementary material available at https://doi. org/10.1186/s12887-021-02996-3.

\section{Additional file 1.}

Additional file 2.

Additional file 3.

\section{Acknowledgements}

We wish to acknowledge with grateful thanks the work of Professor Mark Stevenson in upgrading epiR to version 2.0.11 to enable the analysis of the large integers encountered on this project. We also wish to acknowledge the invaluable support of Professor Giovanni Millo with numerous occasions of technical advice and assistance in relation to the use of the splm software package and geospatial model specification and the spreml function in particular.

\section{Authors' contributions}

ASR assembled the data, designed and conducted the analyses, and wrote the first manuscript draft. GKH provided technical and logistic support, co-wrote the paper, assisted with gaining ethical approval, provided advice on manuscript preparation and general guidance to study conduct. All authors have read and approved the manuscript.

\section{Funding}

No funding was provided for this study. No funding organization played any role in the design and conduct of the study; collection, management, analysis, and interpretation of the data; preparation, review, or approval of the manuscript; and decision to submit the manuscript for publication.

\section{Availability of data and materials}

All data generated or analysed during this study are included in this published article and its supplementary information files. Data has been made publicly available on the Mendeley Database Repository and can be accessed from this URL https://doi.org/10.17632/w6ks529sxd.1.

\section{Declarations}

\section{Ethics approval and consent to participate}

The Human Research Ethics Committee of the University of Western Australia provided ethical approval for the study to be undertaken 7th January 2020 (No. RA/4/20/4724). Consent to participate was not required as the data utilized was derived from publicly available anonymous datasets and no individual identifiable data was utilized.

\section{Consent for publication}

Not applicable.

\section{Competing interests}

The authors declare that they have no competing interests.

\section{Author details}

'Division of Psychiatry, University of Western Australia, Crawley, WA 6009, Australia. ${ }^{2}$ School of Medical and Health Sciences, Edith Cowan University, Joondalup, WA 6027, Australia.

Received: 4 April 2021 Accepted: 3 November 2021

Published online: 19 January 2022

\section{References}

1. Greenwich Biosciences. Epidiolex: Highlights of Prescribing Information. In: Food and Drug Administration, editor. Silver Springs: FDA; 2018. p. 1.

2. Package Leaflet: Information for the Patient Sativex Oromucosal spray. http://www.medicines.org.uk/emc/PIL.23228.latest.pdf.
3. Brown SJ, Mensah FK, Ah Kit J, Stuart-Butler D, Glover K, Leane C, et al. Use of cannabis during pregnancy and birth outcomes in an Aboriginal birth cohort: a cross-sectional, population-based study. BMJ Open. 2016;6(2):e010286.

4. Volkow ND, Compton WM, Wargo EM. The Risks of Marijuana Use During Pregnancy. Jama. 2017;317(2):129-30.

5. Volkow ND, Han B, Compton WM, Blanco C. Marijuana Use During Stages of Pregnancy in the United States. Ann Intern Med. 2017;166(10):763-4.

6. Jenkins KJ, Correa A, Feinstein JA, Botto L, Britt AE, Daniels SR, et al. Noninherited risk factors and congenital cardiovascular defects: current knowledge: a scientific statement from the American Heart Association Council on Cardiovascular Disease in the Young: endorsed by the American Academy of Pediatrics. Circulation. 2007;115(23):2995-3014.

7. Van Gelder MMHJ, Donders ART, Devine O, Roeleveld N, Reefhuis J. Using bayesian models to assess the effects of under-reporting of cannabis use on the association with birth defects, national birth defects prevention study, 1997-2005. Paediatr Perinat Epidemiol. 2014:28(5):424-33.

8. Van Gelder MMHJ, Reefhuis J, Caton AR, Werler MM, Druschel CM, Roeleveld N. Maternal periconceptional illicit drug use and the risk of congenital malformations. Epidemiology. 2009;20(1):60-6.

9. ACOG Committee Opinion: Marijuana Use During Pregnancy and Lactation. https://www.acog.org/en/Clinical/Clinical\%20Guidance/Commi ttee\%20Opinion/Articles/2017/10/Marijuana\%20Use\%20During\%20Pre gnancy\%20and\%20Lactation.

10. Brents L. Correlates and consequences of Prenatal Cannabis Exposure (PCE): Identifying and Characterizing Vulnerable Maternal Populations and Determining Outcomes in Exposed Offspring. In: Preedy VR, editor. Handbook of Cannabis and Related Pathologies: Biology, Pharmacology, Diagnosis and Treatment. Volume 1, edn. London: Academic; 2017. p. 160-70.

11. Geber WF, Schramm LC. Effect of marihuana extract on fetal hamsters and rabbits. Toxicol Appl Pharmacol. 1969;14(2):276-82.

12. Graham JDP. Cannabis and Health. In: Graham JDP, editor. Cannabis and Health. Volume 1. 1st ed. London, New York, San Francisco: Academic; 1976. p. 271-320.

13. Forrester MB, Merz RD. Risk of selected birth defects with prenatal illicit drug use, Hawaii, 1986-2002. J Toxicol Environ Health. 2007:70(1):7-18.

14. Jacobson CB, Berlin CM. Possible reproductive detriment in LSD users. Jama. 1972;222(11):1367-73.

15. Stenchever MA, Kunysz TJ, Allen MA. Chromosome breakage in users of marihuana. Am J Obstet Gynecol. 1974;118(1):106-13.

16. Reece AS, Hulse GK. Contemporary epidemiology of rising atrial septal defect trends across USA 1991-2016: a combined ecological geospatiotemporal and causal inferential study. BMC Pediatr. 2020;20(1):539.

17. Reece AS, Hulse GK. Cannabis Consumption Patterns Explain the EastWest Gradient in Canadian Neural Tube Defect Incidence: An Ecological Study. Glob Pediatr Health. 2019;6:2333794x19894798.

18. Reece AS, Hulse GK. Canadian Cannabis Consumption and Patterns of Congenital Anomalies: An Ecological Geospatial Analysis. J Addict Med. 2020;14(5):e195-210.

19. Reece AS, Hulse GK. Broad Spectrum epidemiological contribution of cannabis and other substances to the teratological profile of northern New South Wales: geospatial and causal inference analysis. BMC Pharmacol Toxicol. 2020;21(1):75

20. Reece AS, Hulse GK. Cannabis Teratology Explains Current Patterns of Coloradan Congenital Defects: The Contribution of Increased Cannabinoid Exposure to Rising Teratological Trends. Clin Pediatr (Phila). 2019;58(10):1085-123.

21. Russo C, Ferk F, Mišík M, Ropek N, Nersesyan A, Mejri D, et al. Low doses of widely consumed cannabinoids (cannabidiol and cannabidivarin) cause DNA damage and chromosomal aberrations in human-derived cells. Arch Toxicol. 2019;93(1):179-88.

22. Zimmerman AM, Zimmerman S, Raj AY. Effects of Cannabinoids on spermatogenesis in mice. In: Nahas GG, Sutin KM, Harvey DJ, Agurell S, editors. Marihuana and medicine. edn. Totowa: Humana Press; 1999. p. 347-58.

23. Mon MJ, Haas AE, Stein JL, Stein GS. Influence of psychoactive and nonpsychoactive cannabinoids on cell proliferation and macromolecular biosynthesis in human cells. Biochem Pharmacol. 1981;30(1):31-43. 
24. Tahir SK, Zimmerman AM. Influence of marihuana on cellular structures and biochemical activities. Pharmacol Biochem Behav. 1991:40(3):617-23.

25. Mon MJ, Haas AE, Stein JL, Stein GS. Influence of psychoactive and nonpsychoactive cannabinoids on chromatin structure and function in human cells. Biochem Pharmacol. 1981;30(1):45-58.

26. Zimmerman AM, Raj AY. Influence of cannabinoids on somatic cells in vivo. Pharmacology. 1980;21(4):277-87.

27. Zimmerman AM, Stich H, San R. Nonmutagenic action of cannabinoids in vitro. Pharmacology. 1978;16(6):333-43.

28. Zimmerman S, Zimmerman AM. Genetic effects of marijuana. Int J Addict. 1990;25(1A):19-33.

29. McClean DK, Zimmerman AM. Action of delta 9-tetrahydrocannabinol on cell division and macromolecular synthesis in division-synchronized protozoa. Pharmacology. 1976;14(4):307-21.

30. Parker SJ, Zuckerman BS, Zimmermann AM. The Effects of Maternal Marijuana Use During Pregnancy on Fetal Growth. In: Nahas GG, Sutin KM, Harvey DJ, Agurell S, editors. Marijuana in Medicine. Volume 1, edn. Totowa, New York: Humana Press; 1999. p. 461-8.

31. Tahir SK, Trogadis JE, Stevens JK, Zimmerman AM. Cytoskeletal organization following cannabinoid treatment in undifferentiated and differentiated PC12 cells. Biochem Cell Biol. 1992;70(10-11):1159-73.

32. Thomas J, Tilak S, Zimmerman S, Zimmerman AM. Action of delta 9-tetrahydrocannabinol on the pool of acid soluble nucleotides. Cytobios. 1984;40(158):71-85

33. Zimmerman AM, Zimmerman S. Cytogenetic Studies of Cannabinoid Effects. In: Braude MC, Zimmerman AM, editors. Genetic and Perinatal Effects of Abused Substances. Volume 1, edn. New York: Academic Press Inc:; Harcourt, Brace Jovanovich; 1987. p. 95-112.

34. Zimmerman AM, Zimmerman S, Raj AY. Effects of Cannabinoids on Spermatogensis in Mice. In: Nahas GG, Sutin KM, Harvey DJ, Agurell S, editors. Marijuana and Medicine. Volume 1. 1st ed. Totowa, New York: Humana Press; 1999. p. 347-58.

35. Takei Y, Yun J, Zheng S, Ollikainen N, Pierson N, White J, et al. Integrated spatial genomics reveals global architecture of single nuclei. Nature. 2021;590(7845):344-50.

36. Hughes V. Epigenetics: The Sins of the Father. Nature. 2014:507(7490):22-4.

37. DiNieri JA, Wang X, Szutorisz H, Spano SM, Kaur J, Casaccia P, et al. Maternal cannabis use alters ventral striatal dopamine D2 gene regulation in the offspring. Biol Psychiatry. 2011;70(8):763-9.

38. Szutorisz H, DiNieri JA, Sweet E, Egervari G, Michaelides M, Carter JM, et al. Parental THC exposure leads to compulsive heroin-seeking and altered striatal synaptic plasticity in the subsequent generation. Neuropsychopharmacology. 2014;39(6):1315-23.

39. Szutorisz H, Hurd YL. Epigenetic Effects of Cannabis Exposure. Biol Psychiatry. 2016;79(7):586-94

40. Szutorisz H, Hurd YL. High times for cannabis: Epigenetic imprint and its legacy on brain and behavior. Neurosci Biobehav Rev. 2018;85:93-101.

41. Watson CT, Szutorisz H, Garg P, Martin Q, Landry JA, Sharp AJ, et al. Genome-Wide DNA Methylation Profiling Reveals Epigenetic Changes in the Rat Nucleus Accumbens Associated With Cross-Generational Effects of Adolescent THC Exposure. Neuropsychopharmacology. 2015;40(13):2993-3005.

42. Fish EW, Murdaugh LB, Zhang C, Boschen KE, Boa-Amponsem O, Mendoza-Romero HN, et al. Cannabinoids Exacerbate Alcohol Teratogenesis by a CB1-Hedgehog Interaction. Sci Rep. 2019;9(1):16057.

43. Bermejo-Sanchez E, Cuevas L, Amar E, Bakker MK, Bianca S, Bianchi F, et al. Amelia: a multi-center descriptive epidemiologic study in a large dataset from the International Clearinghouse for Birth Defects Surveillance and Research, and overview of the literature. Am J Med Genet C Semin Med Genet. 2011;157C(4):288-304.

44. Bermejo-Sanchez E, Cuevas L, Amar E, Bianca S, Bianchi F, Botto LD, et al. Phocomelia: a worldwide descriptive epidemiologic study in a large series of cases from the International Clearinghouse for Birth Defects Surveillance and Research, and overview of the literature. Am J Med Genet C Semin Med Genet. 2011;157C(4):305-20.

45. Babies born with deformed hands spark investigation in Germany. https://edition.cnn.com/2019/09/16/health/hand-deformitiesbabies-gelsenkirchen-germany-intl-scli-grm/index.html.
46. Le Figaro with AFP. Ain: seizure of $135 \mathrm{~kg}$ of cannabis. Le Figaro [Internet]. 2018 [cited 2018 3rd Novermber 2018]. Available from: http:// www.lefigaro.fr/flash-actu/2018/10/18/97001-20181018FILWWW0 0290-ain-saisie-de-135kg-de-cannabis.php.

47. Willsher K. Baby arm defects prompt nationwide investigation in France. In: Guardian. London: The Guardian; 2018.

48. Agence France-Presse in Paris. France to investigate cause of upper limb defects in babies. In: The Guardian. London: The Guardian; 2018.

49. Connexion Journalist. Mayor wants to grow and sell cannabis in French fields. 2018. [cited 2018 3rd November 2018]. Available from: https://www.connexionfrance.com/Frenchnews/legalisation-ofcannabis-in-France.

50. Gant J. Scientists are baffled by spatter of babies born without hands or arms in France, as investigation fails to discover a cause. In: Daily Mail. vol. Sunday 14th July. London: Daily Mail; 2019.

51. McBride WG. Thalidomide and Congenital Malformations. Lancet. 1962;2:1358-9.

52. Zhou S, Wang F, Hsieh TC, Wu JM, Wu E. Thalidomide-a notorious sedative to a wonder anticancer drug. Curr Med Chem. 2013:20(33):4102-8.

53. Rehman W, Arfons LM, Lazarus HM. The rise, fall and subsequent triumph of thalidomide: lessons learned in drug development. Ther Adv Hematol. 2011;2(5):291-308.

54. Price PJ, Suk WA, Spahn GJ, Freeman AE. Transformation of Fischer rat embryo cells by the combined action of murine leukemia virus and (-)-trans- 9 -tetrahydrocannabinol. Proc Soc Exp Biol Med. 1972;140(2):454-6.

55. Busch FW, Seid DA, Wei ET. Mutagenic activity of marihuana smoke condensates. Cancer Lett. 1979;6(6):319-24.

56. Vela G, Martin S, Garcia-Gil L, Crespo JA, Ruiz-Gayo M, Fernandez-Ruiz $J$ J, et al. Maternal exposure to delta9-tetrahydrocannabinol facilitates morphine self-administration behavior and changes regional binding to central mu opioid receptors in adult offspring female rats. Brain Res. 1998;807(1-2):101-9.

57. Sarafian TA, Kouyoumjian S, Khoshaghideh F, Tashkin DP, Roth MD. Delta 9-tetrahydrocannabinol disrupts mitochondrial function and cell energetics. Am J Physiol. 2003;284(2):L298-306.

58. Sarafian TA, Habib N, Oldham M, Seeram N, Lee RP, Lin L, et al. Inhaled marijuana smoke disrupts mitochondrial energetics in pulmonary epithelial cells in vivo. Am J Physiol. 2006;290(6):L1202-9.

59. Morimoto S, Tanaka Y, Sasaki K, Tanaka H, Fukamizu T, Shoyama Y, et al. Identification and characterization of cannabinoids that induce cell death through mitochondrial permeability transition in Cannabis leaf cells. J Biol Chem. 2007;282(28):20739-51.

60. Shoyama Y, Sugawa C, Tanaka H, Morimoto S. Cannabinoids act as necrosis-inducing factors in Cannabis sativa. Plant Signal Behav. 2008;3(12):1111-2.

61. Fisar Z, Singh N, Hroudova J. Cannabinoid-induced changes in respiration of brain mitochondria. Toxicol Lett. 2014;231(1):62-71.

62. Koller VJ, Auwarter V, Grummt T, Moosmann B, Misik M, Knasmuller S. Investigation of the in vitro toxicological properties of the synthetic cannabimimetic drug CP-47,497-C8. Toxicol Appl Pharmacol. 2014;277(2):164-71.

63. Koller VJ, Ferk F, Al-Serori H, Misik M, Nersesyan A, Auwarter V, et al. Genotoxic properties of representatives of alkylindazoles and aminoalkyl-indoles which are consumed as synthetic cannabinoids. Food Chem Toxicol. 2015;80:130-6.

64. Singh N, Hroudova J, Fisar Z. Cannabinoid-Induced Changes in the Activity of Electron Transport Chain Complexes of Brain Mitochondria. J Mol Neurosci. 2015;56(4):926-31.

65. Russo C, Ferk F, Misik M, Ropek N, Nersesyan A, Mejri D, et al. Low doses of widely consumed cannabinoids (cannabidiol and cannabidivarin) cause DNA damage and chromosomal aberrations in human-derived cells. Arch Toxicol. 2019:93(1):179-88.

66. Reece AS, Hulse GK. Effect of Cannabis Legalization on US Autism Incidence and Medium Term Projections. Clini Pediatr Open Access. 2019:4(2):1-17

67. VanderWeele TJ, Ding P, Mathur M. Technical Considerations in the Use of the E-Value. J Causal Inference. 2019;7(2):1-11.

68. VanderWeele TJ, Ding P. Sensitivity Analysis in Observational Research: Introducing the E-Value. Ann Intern Med. 2017;167(4):268-74. 
69. VanderWeele TJ, Mathur MB. Commentary: Developing bestpractice guidelines for the reporting of E-values. Int J Epidemiol. 2020;49(5):1495-7.

70. NBDPN. Major Birth Defects Data from Population-based Birth Defects Surveillance Programs in the United States, 2011-2015. In: Edited by Network NBDP, vol. NBDPN, CDC. NBDPN, Centers for Disease Control. Atlanta: National Birth Defects Prevention Network; 2018.

71. Substance Abuse and Mental Health Data Archive (SAMHDA). https:// www.datafiles.samhsa.gov/.

72. EISohly MA, Mehmedic Z, Foster S, Gon C, Chandra S, Church JC. Changes in Cannabis Potency Over the Last 2 Decades (19952014): Analysis of Current Data in the United States. Biol Psychiatry. 2016:79(7):613-9.

73. Chandra S, Radwan MM, Majumdar CG, Church JC, Freeman TP, EISohly MA. New trends in cannabis potency in USA and Europe during the last decade (2008-2017). Eur Arch Psychiatry Clin Neurosci. 2019;269(1):5-15.

74. ElSohly MA, Ross SA, Mehmedic Z, Arafat R, Yi B, Banahan BF 3rd Potency trends of delta9-THC and other cannabinoids in confiscated marijuana from 1980-1997. J Forensic Sci. 2000:45(1):24-30.

75. Bird TM, Hobbs CA, Cleves MA, Tilford JM, Robbins JM. National rates of birth defects among hospitalized newborns. Birth Defects Res A Clin Mol Teratol. 2006;76(11):762-9.

76. Natoli JL, Ackerman DL, McDermott S, Edwards JG. Prenatal diagnosis of Down syndrome: a systematic review of termination rates (1995-2011). Prenat Diagn. 2012;32(2):142-53.

77. Mansfield C, Hopfer S, Marteau TM. Termination rates after prenatal diagnosis of Down syndrome, spina bifida, anencephaly, and Turner and Klinefelter syndromes: a systematic literature review. European Concerted Action: DADA (Decision-making After the Diagnosis of a fetal Abnormality). Prenat Diagn. 1999;19(9):808-12.

78. Abeywardana S, Sullivan EA. Congenital Anomalies in Australia, 20022003. In: Edited by Australian Institute of Health and Welfare PSU, vol. Australian Institute of Health and Welfare. Sydney: Australian Institute of Health and Welfare; 2008.

79. Brick DH, Allan LD. Outcome of prenatally diagnosed congenital heart disease: an update. Pediatr Cardiol. 2002;23(4):449-53.

80. Howell S, Endo T, MacLeod S, Cornes S. Congenital Anomalies in Queensland: 1 July 2007 to 30 June 2010. Statist Anal Rep \#1. 2011:1(1):1-22.

81. Siljee JE, Knegt AC, Knapen MF, Bekker MN, Visser GH, Schielen PC. Positive predictive values for detection of trisomies 21, 18 and 13 and termination of pregnancy rates after referral for advanced maternal age, first trimester combined test or ultrasound abnormalities in a national screening programme (2007-2009). Prenat Diagn. 2014;34(3):259-64.

82. Tararbit K, Bui TT, Lelong N, Thieulin AC, Goffinet F, Khoshnood B. Clinical and socioeconomic predictors of pregnancy termination for fetuses with congenital heart defects: a population-based evaluation. Prenat Diagn. 2013;33(2):179-86.

83. Women and Newborn Health Service, Department of Health, Government of Western Australia. Western Australian Register of Developmental Anomalies 1980-2014. In: Edited by Western Australia Health, vol. 1, vol. 28. Perth: Western Australia Health; 2015.

84. tidycensus: Load US Census Boundary and Attribute Data as 'tidyverse' and 'sf'-Ready Data Frames. https://www.r-pkg.org/pkg/tidycensus; https://cran.rstudio.com/web/packages/tidycensus/tidycensus.pdf.

85. National Survey of Drug Use and Health (NSDUH 2018). https://www. datafiles.samhsa.gov/study/national-survey-drug-use-and-healthnsduh-2018-nid18757\#.

86. Pearl J, Mackaenzie D. The Book of Why. The New Science of Cause and Effect, vol. 1. New York: Basic Books; 2019.

87. Hölzel BN, Pfannkuche K, Allner B, Allner HT, Hescheler J, Derichsweiler $D$, et al. Following the adverse outcome pathway from micronucleus to cancer using H2B-eGFP transgenic healthy stem cells. Arch Toxicol. 2020;94(9):3265-80.

88. Reece AS, Hulse GK. Epidemiological Associations of Various Substances and Multiple Cannabinoids with Autism in USA. Clin Pediatr Open Access. 2019;4(2):1-20.

89. Reece AS, Hulse GK. Epidemiological Overview of Multidimensional Chromosomal and Genome Toxicity of Cannabis Exposure in Congenital Anomalies and Cancer Development. Sci Rep. 2021;11(1):13892.
90. Short TD, Stallings EB, Isenburg J, O'Leary LA, Yazdy MM, Bohm MK, et al. Gastroschisis Trends and Ecologic Link to Opioid Prescription Rates - United States, 2006-2015. MMWR Morb Mortal Wkly Rep. 2019;68(2):31-6.

91. Reece AS, Wang W, Hulse GK. Pathways from epigenomics and glycobiology towards novel biomarkers of addiction and its radical cure. Med Hypotheses. 2018;116:10-21.

92. Carlson BM. Human Embryology and Developmental Biology, vol. 1. 6th ed. Philadelphia: Elsevier; 2019.

93. Dyer LA, Kirby ML. Sonic hedgehog maintains proliferation in secondary heart field progenitors and is required for normal arterial pole formation. Dev Biol. 2009;330(2):305-17.

94. Christ A, Marczenke M, Willnow TE. LRP2 controls sonic hedgehogdependent differentiation of cardiac progenitor cells during outflow tract formation. Hum Mol Genet. 2020;29(19):3183-96.

95. Yamagishi H, Maeda J, Hu T, McAnally J, Conway SJ, Kume T, et al. Tbx1 is regulated by tissue-specific forkhead proteins through a common Sonic hedgehog-responsive enhancer. Genes Dev. 2003;17(2):269-81.

96. Hutson MR, Sackey FN, Lunney K, Kirby ML. Blocking hedgehog signaling after ablation of the dorsal neural tube allows regeneration of the cardiac neural crest and rescue of outflow tract septation. Dev Biol. 2009;335(2):367-73.

97. Koefoed K, Skat-Rørdam J, Andersen P, Warzecha CB, Pye M, Andersen TA, et al. The E3 ubiquitin ligase SMURF1 regulates cell-fate specification and outflow tract septation during mammalian heart development. Sci Rep. 2018;8(1):9542.

98. Maynard TM, Gopalakrishna D, Meechan DW, Paronett EM, Newbern JM, LaMantia A-S. 22q11 Gene dosage establishes an adaptive range for sonic hedgehog and retinoic acid signaling during early development. Hum Mol Genet. 2013;22(2):300-12.

99. Bhatt S, Diaz R, Trainor PA. Signals and switches in Mammalian neural crest cell differentiation. Cold Spring Harb Perspect Biol. 2013;5(2):a008326.

100. Sherif HMF. Heterogeneity in the Segmental Development of the Aortic Tree: Impact on Management of Genetically Triggered Aortic Aneurysms. Aorta (Stamford). 2014;2(5):186-95.

101. Kolesová H, Roelink H, Grim M. Sonic hedgehog is required for the assembly and remodeling of branchial arch blood vessels. Dev Dyn. 2008;237(7):1923-34.

102. Kim JD, Kang H, Larrivée B, Lee MY, Mettlen M, Schmid SL, et al. Context-dependent proangiogenic function of bone morphogenetic protein signaling is mediated by disabled homolog 2. Dev Cell. 2012;23(2):441-8

103. Roman BL, Pekkan K. Mechanotransduction in embryonic vascular development. Biomech Model Mechanobiol. 2012;11(8):1149-68.

104. Geber WF, Schramm LC. Teratogenicity of marihuana extract as influenced by plant origin and seasonal variation. Arch Int Pharmacodyn Ther. 1969;177(1):224-30.

105. Reece AS, Hulse GK. Gastroschisis and Autism-Dual Canaries in the Californian Coalmine. JAMA Surg. 2019;154(4):366-7.

106. Porath AJ, Fried PA. Effects of prenatal cigarette and marijuana exposure on drug use among offspring. Neurotoxicol Teratol. 2005;27(2):267-77.

107. Fried PA, Watkinson B, Gray R. Neurocognitive consequences of marihuana--a comparison with pre-drug performance. Neurotoxicol Teratol. 2005:27(2):231-9.

108. Fried P, Watkinson B, James D, Gray R. Current and former marijuana use: preliminary findings of a longitudinal study of effects on $\mathrm{IQ}$ in young adults. CMAJ. 2002;166(7):887-91.

109. Fried PA, Smith AM. A literature review of the consequences of prenatal marihuana exposure. An emerging theme of a deficiency in aspects of executive function. Neurotoxicol Teratol. 2001;23(1):1-11.

110. Smith AM, Mioduszewski O, Hatchard T, Byron-Alhassan A, Fall C, Fried PA. Prenatal marijuana exposure impacts executive functioning into young adulthood: An fMRI study. Neurotoxicol Teratol. 2016;58:53-9.

111. Smith AM, Longo CA, Fried PA, Hogan MJ, Cameron I. Effects of marijuana on visuospatial working memory: an fMRI study in young adults. Psychopharmacology. 2010;210(3):429-38.

112. Smith AM, Fried PA, Hogan MJ, Cameron I. Effects of prenatal marijuana on visuospatial working memory: an fMRI study in young adults. Neurotoxicol Teratol. 2006;28(2):286-95. 
113. Reece AS, Hulse GK. Cannabis in Pregnancy - Rejoinder, Exposition and Cautionary Tales. Psychiatr Times. 2020; https://www.bing.com/search? $\mathrm{q}=$ Cannabis+in+Pregnancy+\%E2\%80\%93+Rejoinder\%82C+Expos ition+and+Cautionary+Tales\&cvid $=22538 \mathrm{e} 20124 \mathrm{c} 04711 \mathrm{~b} 9201748$ 9c92063214a\&aqs=edge..92017469i92017457.92017439j92017480j92 $017481 \& p g \mid t=92017443 \& F O R M=A N S P A 92017481 \& P C=U 92017531$.

114. David AL, Holloway A, Thomasson L, Syngelaki A, Nicolaides K, Patel RR, et al. A case-control study of maternal periconceptual and pregnancy recreational drug use and fetal malformation using hair analysis. PLoS One. 2014;9(10):e111038.

115. Draper ES, Rankin J, Tonks AM, Abrams KR, Field DJ, Clarke M, et al. Recreational drug use: a major risk factor for gastroschisis? Am J Epidemiol. 2008;167(4):485-91.

116. Skarsgard ED, Meaney C, Bassil K, Brindle M, Arbour L, Moineddin R, et al. Maternal risk factors for gastroschisis in Canada. Birth Defects Res A Clin Mol Teratol. 2015;103(2):111-8.

117. Torfs CP, Velie EM, Oechsli FW, Bateson TF, Curry CJ. A population-based study of gastroschisis: demographic, pregnancy, and lifestyle risk factors. Teratology. 1994;50(1):44-53.

118. Werler MM, Sheehan JE, Mitchell AA. Association of vasoconstrictive exposures with risks of gastroschisis and small intestinal atresia. Epidemiology. 2003;14(3):349-54.

119. Mon MJ, Jansing RL, Doggett S, Stein JL, Stein GS. Influence of delta9tetrahydrocannabinol on cell proliferation and macromolecular biosynthesis in human cells. Biochem Pharmacol. 1978;27(13):1759-65.

120. Jakubovič A, McGeer PL, Fitzsimmons RC. Effects of $\triangle 9$-tetrahydrocannabinol and ethanol on body weight protein and nucleic acid synthesis in chick embryos. J Toxicol Environ Health. 1976;1(3):441-7.

121. Blevins RD, Regan JD. delta-9-Tetrahydrocannabinol: effect on macromolecular synthesis in human and other mammalian cells. Arch Toxicol. 1976;35(2):127-35.

122. Nahas GG, Morishima A, Desoize B. Effects of cannabinoids on macromolecular synthesis and replication of cultured lymphocytes. Fed Proc. 1977;36(5):1748-52.

123. Pacher P, Steffens S, Hasko G, Schindler TH, Kunos G. Cardiovascular effects of marijuana and synthetic cannabinoids: the good, the bad, and the ugly. Nat Rev Cardiol. 2018;15(3):151-66.

124. Molica F, Burger F, Thomas A, Staub C, Tailleux A, Staels B, et al. Endogenous cannabinoid receptor $\mathrm{CB} 1$ activation promotes vascular smooth-muscle cell proliferation and neointima formation. J Lipid Res. 2013;54(5):1360-8.

125. Slavic S, Lauer D, Sommerfeld M, Kemnitz UR, Grzesiak A, Trappiel M, et al. Cannabinoid receptor 1 inhibition improves cardiac function and remodelling after myocardial infarction and in experimental metabolic syndrome. J Mol Med (Berl). 2013;91(7):811-23.

126. Jouanjus E, Lapeyre-Mestre M, Micallef J, French Association of the Regional A, Dependence Monitoring Centres Working Group on Cannabis C. Cannabis use: signal of increasing risk of serious cardiovascular disorders. J Am Heart Assoc. 2014;3(2):e000638.

127. Stanley C, O'Sullivan SE. Vascular targets for cannabinoids: animal and human studies. Br J Pharmacol. 2014;171(6):1361-78.

128. Folkerth RD, Habbe DM, Boyd TK, McMillan K, Gromer J, Sens MA, et al. Gastroschisis, destructive brain lesions, and placental infarction in the second trimester suggest a vascular pathogenesis. Pediatr Dev Pathol. 2013;16(5):391-6.

129. Hoyme HE, Higginbottom MC, Jones KL. The vascular pathogenesis of gastroschisis: intrauterine interruption of the omphalomesenteric artery. J Pediatr. 1981;98(2):228-31.

130. Lubinsky M. A vascular and thrombotic model of gastroschisis. Am J Med Genet A. 2014;164A(4):915-7.

131. Pistor G, Marzheuser-Brands S, Weber G, Streich R. Intraoperative vascular assessment for estimation of risk in primary closure of omphalocele and gastroschisis. Pediatr Surg Int. 1996;11(2-3):86-90.

132. Van Allen MI, Smith DW. Vascular pathogenesis of gastroschisis. J Pediatr. 1981;98(4):662-3.

133. Werler MM, Mitchell AA, Moore CA, Honein MA, National Birth Defects Prevention S. Is there epidemiologic evidence to support vascular disruption as a pathogenesis of gastroschisis? Am J Med Genet A. 2009;149A(7):1399-406.

134. Ngan ESW, Kim KH, Hui CC. Sonic Hedgehog Signaling and VACTERL Association. Mol Syndromol. 2013;4(1-2):32-45.
135. Ryckebüsch L, Bertrand N, Mesbah K, Bajolle F, Niederreither K, Kelly RG, et al. Decreased levels of embryonic retinoic acid synthesis accelerate recovery from arterial growth delay in a mouse model of DiGeorge syndrome. Circ Res. 2010;106(4):686-94.

136. Reece AS, Hulse GK. Rapid Response to Lane. Re: Cannabis exposure as an interactive cardiovascular risk factor and accelerant of organismal ageing: a longitudinal study, 2016. BMJ Open. 2020;6:e011891-902.

137. Reece AS, Hulse GK. Response to Polocaro and Vettraino. Mo Med. 2020;117(6) In Press.

138. Reece AS, Hulse GK. Chromothripsis and epigenomics complete causality criteria for cannabis- and addiction-connected carcinogenicity, congenital toxicity and heritable genotoxicity. Mutat Res. 2016;789:15-25.

139. Reece AS, Hulse GK. Impacts of Cannabinoid Epigenetics on Human Development: Reflections on Murphy et. al. 'Cannabinoid Exposure and Altered DNA Methylation in Rat and Human Sperm' Epigenetics 2018; 13: 1208-1221. Epigenetics. 2019:1-16.

140. Murphy SK, Itchon-Ramos N, Visco Z, Huang Z, Grenier C, Schrott R, et al. Cannabinoid exposure and altered DNA methylation in rat and human sperm. Epigenetics. 2018;13(12):1208-21.

141. Wilson RG Jr, Tahir SK, Mechoulam R, Zimmerman S, Zimmerman AM. Cannabinoid enantiomer action on the cytoarchitecture. Cell Biol Int. 1996;20(2):147-57.

142. Reece AS, Hulse GK. Impact of Lifetime Opioid Exposure on Arterial Stiffness and Vascular Age: Cross-sectional and Longitudinal Studies in Men and Women. BMJ Open. 2014;4(6):1-19.

143. Tyser RCV, Ibarra-Soria X, McDole K, Arcot Jayaram S, Godwin J, van den Brand TAH, et al. Characterization of a common progenitor pool of the epicardium and myocardium. Science. 2021;371(6533):eabb2986.

144. Abramyan J. Hedgehog Signaling and Embryonic Craniofacial Disorders. J Dev Biol. 2019;7(2):9.

145. Carlson BM. Human Embryology and Developmental Biology, vol. 1. 5th ed. Philadelphia: Elsevier; 2014.

146. Ing GM, Olman CL, Oyd JR. Drug-Induced (Thalidomide) Malformations. Can Med Assoc J. 1962;87(24):1259-62.

147. Kizaki M, Hashimoto Y. New tubulin polymerization inhibitor derived from thalidomide: implications for anti-myeloma therapy. Curr Med Chem. 2008;15(8):754-65.

148. Iguchi T, Yachide-Noguchi T, Hashimoto Y, Nakazato S, Sagawa M, Ikeda Y, et al. Novel tubulin-polymerization inhibitor derived from thalidomide directly induces apoptosis in human multiple myeloma cells: possible antimyeloma mechanism of thalidomide. Int J Mol Med. 2008;21(2):163-8.

149. Tseng S, Pak G, Washenik K, Pomeranz MK, Shupack JL. Rediscovering thalidomide: a review of its mechanism of action, side effects, and potential uses. J Am Acad Dermatol. 1996;35(6):969-79.

150. Therapontos C, Erskine L, Gardner ER, Figg WD, Vargesson N. Thalidomide induces limb defects by preventing angiogenic outgrowth during early limb formation. Proc Natl Acad Sci U S A. 2009;106(21):8573-8.

151. Vargesson N. Thalidomide-induced limb defects: resolving a 50 -year-old puzzle. Bioessays. 2009;31(12):1327-36.

152. Vargesson N. Thalidomide-induced teratogenesis: history and mechanisms. Birth Defects Res C Embryo Today. 2015;105(2):140-56.

153. McBride W. Health of thalidomide victims and their progeny. Lancet. 2004;363(9403):169.

154. Franks ME, Macpherson GR, Figg WD. Thalidomide. Lancet. 2004;363(9423):1802-11.

155. Linz W. Thalidomide and Congenital Abnormalities. Lancet. 1962;7223(i):271-2.

156. Melchert M, List A. The thalidomide saga. Int J Biochem Cell Biol. 2007;39(7-8):1489-99.

157. Aguado T, Romero E, Monory K, Palazuelos J, Sendtner M, Marsicano $\mathrm{G}$, et al. The CB1 cannabinoid receptor mediates excitotoxicityinduced neural progenitor proliferation and neurogenesis. J Biol Chem. 2007;282(33):23892-8.

158. Williams EJ, Walsh FS, Doherty P. The FGF receptor uses the endocannabinoid signaling system to couple to an axonal growth response. J Cell Biol. 2003;160(4):481-6.

159. Asimaki O, Leondaritis G, Lois G, Sakellaridis N, Mangoura D. Cannabinoid 1 receptor-dependent transactivation of fibroblast growth factor receptor 1 emanates from lipid rafts and amplifies extracellular 
signal-regulated kinase 1/2 activation in embryonic cortical neurons. J Neurochem. 2011;116(5):866-73.

160. Birerdinc A, Jarrar M, Stotish T, Randhawa M, Baranova A. Manipulating molecular switches in brown adipocytes and their precursors: a therapeutic potential. Prog Lipid Res. 2013;52(1):51-61.

161. Richard D, Picard F. Brown fat biology and thermogenesis. Front Biosci (Landmark Ed). 2011;16:1233-60.

162. Xu TR, Yang Y, Ward R, Gao L, Liu Y. Orexin receptors: multi-functional therapeutic targets for sleeping disorders, eating disorders, drug addiction, cancers and other physiological disorders. Cell Signal. 2013:25(12):2413-23.

163. Fraher D, Ellis MK, Morrison S, McGee SL, Ward AC, Walder K, et al. Lipid Abundance in Zebrafish Embryos Is Regulated by Complementary Actions of the Endocannabinoid System and Retinoic Acid Pathway. Endocrinology. 2015;156(10):3596-609.

164. Kučukalić S, Ferić Bojić E, Babić R, Avdibegović E, Babić D, Agani F, et al. Genetic Susceptibility to Posttraumatic Stress Disorder: Analyses of the Oxytocin Receptor, Retinoic Acid Receptor-Related Orphan Receptor A and Cannabinoid Receptor 1 Genes. Psychiatr Danub. 2019;31(2):219-26.

165. Lee YS, Jeong WI. Retinoic acids and hepatic stellate cells in liver disease. J Gastroenterol Hepatol. 2012;27(Suppl 2):75-9.

166. Frampton G, Coufal M, Li H, Ramirez J, DeMorrow S. Opposing actions of endocannabinoids on cholangiocarcinoma growth is via the differential activation of Notch signaling. Exp Cell Res. 2010;316(9):1465-78.

167. Kim D, Lim S, Park M, Choi J, Kim J, Han H, et al. Ubiquitinationdependent CARM1 degradation facilitates Notch1-mediated podocyte apoptosis in diabetic nephropathy. Cell Signal. 2014;26(9):1774-82.

168. Lu T, Newton C, Perkins I, Friedman H, Klein TW. Cannabinoid treatment suppresses the T-helper cell-polarizing function of mouse dendritic cells stimulated with Legionella pneumophila infection. J Pharmacol Exp Ther. 2006;319(1):269-76.

169. Newton CA, Chou PJ, Perkins I, Klein TW. CB(1) and CB(2) cannabinoid receptors mediate different aspects of delta-9-tetrahydrocannabinol (THC)-induced Thelper cell shift following immune activation by Legionella pneumophila infection. J Neurolmmune Pharmacol. 2009:4(1):92-102.

170. Niu F, Zhao S, Xu CY, Sha H, Bi GB, Chen L, et al. Potentiation of the antitumor activity of adriamycin against osteosarcoma by cannabinoid WIN-55,212-2. Oncol Lett. 2015;10(4):2415-21.

171. Tanveer R, Gowran A, Noonan J, Keating SE, Bowie AG, Campbell VA. The endocannabinoid, anandamide, augments Notch-1 signaling in cultured cortical neurons exposed to amyloid-beta and in the cortex of aged rats. J Biol Chem. 2012;287(41):34709-21.

172. Xapelli S, Agasse F, Sarda-Arroyo L, Bernardino L, Santos T, Ribeiro FF, et al. Activation of type 1 cannabinoid receptor (CB1R) promotes neurogenesis in murine subventricular zone cell cultures. PLoS One. 2013;8(5):e63529.

173. Proto MC, Fiore D, Piscopo C, Franceschelli S, Bizzarro V, Laezza C, et al. Inhibition of Wnt/beta-Catenin pathway and Histone acetyltransferase activity by Rimonabant: a therapeutic target for colon cancer. Sci Rep. 2017;7(1):11678

174. Vallee A, Lecarpentier Y, Guillevin R, Vallee JN. Effects of cannabidiol interactions with Wnt/beta-catenin pathway and PPARgamma on oxidative stress and neuroinflammation in Alzheimer's disease. Acta Biochim Biophys Sin Shanghai. 2017:49(10):853-66.

175. Nallathambi R, Mazuz M, Namdar D, Shik M, Namintzer D, Vinayaka AC, et al. Identification of Synergistic Interaction Between CannabisDerived Compounds for Cytotoxic Activity in Colorectal Cancer Cell Lines and Colon Polyps That Induces Apoptosis-Related Cell Death and Distinct Gene Expression. Cannabis Cannabinoid Res. 2018;3(1):120-35.

176. Petko J, Tranchina T, Patel G, Levenson R, Justice-Bitner S. Identifying novel members of the Wntless interactome through genetic and candidate gene approaches. Brain Res Bull. 2018;138:96-105.

177. Xian X, Tang L, Wu C, Huang L. miR-23b-3p and miR-130a-5p affect cell growth, migration and invasion by targeting CB1R via the Wnt/betacatenin signaling pathway in gastric carcinoma. Onco Targets Ther. 2018;11:7503-12.

178. McKenzie MG, Cobbs LV, Dummer PD, Petros TJ, Halford MM, Stacker SA, et al. Non-canonical Wnt Signaling through Ryk Regulates the Generation of Somatostatin- and Parvalbumin-Expressing Cortical Interneurons. Neuron. 2019;103(5):853-864 e854.
179. Nalli Y, Dar MS, Bano N, Rasool JU, Sarkar AR, Banday J, et al. Analyzing the role of cannabinoids as modulators of Wnt/beta-catenin signaling pathway for their use in the management of neuropathic pain. Bioorg Med Chem Lett. 2019;29(9):1043-6.

180. Foldy C, Malenka RC, Sudhof TC. Autism-associated neuroligin-3 mutations commonly disrupt tonic endocannabinoid signaling. Neuron. 2013;78(3):498-509.

181. Radyushkin K, Hammerschmidt K, Boretius S, Varoqueaux F, El-Kordi A, Ronnenberg A, et al. Neuroligin-3-deficient mice: model of a monogenic heritable form of autism with an olfactory deficit. Genes Brain Behav. 2009;8(4):416-25.

182. Sun C, Zhang L, Chen G. An unexpected role of neuroligin-2 in regulating KCC2 and GABA functional switch. Mol Brain. 2013;6:23.

183. Alpar A, Tortoriello G, Calvigioni D, Niphakis MJ, Milenkovic I, Bakker J, et al. Endocannabinoids modulate cortical development by configuring Slit2/Robo1 signalling. Nat Commun. 2014;5:4421.

184. Kangsamaksin T, Murtomaki A, Kofler NM, Cuervo H, Chaudhri RA, Tattersall IW, et al. NOTCH decoys that selectively block DLL/NOTCH or JAG/NOTCH disrupt angiogenesis by unique mechanisms to inhibit tumor growth. Cancer Discov. 2015;5(2):182-97.

185. London NR, Li DY. Robo4-dependent Slit signaling stabilizes the vasculature during pathologic angiogenesis and cytokine storm. Curr Opin Hematol. 2011;18(3):186-90.

186. Cardenas A, Villalba A, de Juan RC, Pico E, Kyrousi C, Tzika AC, et al. Evolution of Cortical Neurogenesis in Amniotes Controlled by Robo Signaling Levels. Cell. 2018;174(3):590-606 e521.

187. Hill AB. The Environment and Disease: Association or Causation? Proc R Soc Med. 1965;58:295-300.

\section{Publisher's Note}

Springer Nature remains neutral with regard to jurisdictional claims in published maps and institutional affiliations.

Ready to submit your research? Choose BMC and benefit from:

- fast, convenient online submission

- thorough peer review by experienced researchers in your field

- rapid publication on acceptance

- support for research data, including large and complex data types

- gold Open Access which fosters wider collaboration and increased citations

- maximum visibility for your research: over $100 \mathrm{M}$ website views per year

At BMC, research is always in progress.

Learn more biomedcentral.com/submissions 